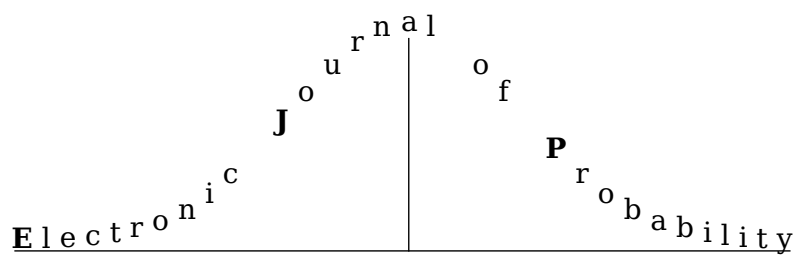

Electron. J. Probab. 25 (2020), article no. 57, 1-56.

ISSN: 1083-6489 https://doi.org/10.1214/20-EJP452

\title{
Rescaling the spatial Lambda-Fleming-Viot process and convergence to super-Brownian motion*
}

\author{
J. Theodore $\operatorname{Cox}^{\dagger} \quad$ Edwin A. Perkins ${ }^{\ddagger}$
}

\begin{abstract}
We show that a space-time rescaling of the spatial Lamba-Fleming-Viot process of Barton and Etheridge converges to super-Brownian motion. This can be viewed as an extension of a result of Chetwynd-Diggle and Etheridge [5]. In that work the scaled impact factors (which govern the event based dynamics) vanish in the limit, here we drop that requirement. The analysis is particularly interesting in the biologically relevant two-dimensional case.
\end{abstract}

Keywords: super-Brownian motion; spatial lambda-Fleming-Viot process. AMS MSC 2010: Primary 60J68; 60K35, Secondary 92D25. Submitted to EJP on September 3, 2019, final version accepted on April 7, 2020. Supersedes arXiv: 1909.03277.

\section{Introduction}

Our purpose in this paper is to extend a result in [5] which shows that certain suitably rescaled spatial Lambda-Fleming-Viot (SLFV) processes converge weakly to super-Brownian motion (SBM). Our extension is analogous to that of allowing nearest neighbour interactions in interacting particle models, as opposed to taking long range limits, and is particularly delicate in the critical two-dimensional case. SBM is a well known measure-valued diffusion, introduced in [26] and [9], for which there is an extensive research literature (e.g., for reviews see [10], [13] and [22]). SLFV processes were introduced more recently, in [12], to serve as models for the evolution of allele frequencies in populations distributed across spatial continua. An analytic construction was given in [2], along with a discussion of the biological significance of the model. A more probabilistic construction was given in [25], one which gives a very useful connection between SLFV processes and their duals. Following [5], we consider here a neutral two-type version of the general SLFV model, taking "space" to be $\mathbb{R}^{d}$. Informally, our process (constructed below) is a Markov process $\left(\mu_{t}\right)_{t \geq 0}$ where for each $x \in \mathbb{R}^{d}$,

\footnotetext{
${ }^{*}$ Research supported in part by an NSRC Discovery Grant

${ }^{\dagger}$ Syracuse University, United States of America. E-mail: jtcox@syr.edu

${ }^{\ddagger}$ The University of British Columbia, Canada. E-mail: perkins@math. ubc.ca
} 
$\mu_{t}(x)$ is a probability distribution on the type space $\{0,1\}$, with the interpretation that $\int_{B} \mu_{t}(x)(\{i\}) d x$ represents the proportion of the population of type $i$ in a region $B \subset \mathbb{R}^{d}$ at time $t$. We will consider an extension of the fixed radius case from [5] (Theorem 2.6 of that reference) and not the interesting variable radius case, also discussed there in Theorem 2.7, in which stable branching arises in the limit.

SBM arises as the limit under Brownian space-time rescaling of a range of critical spatially interacting models in mathematical physics and biology above the critical dimension including critical oriented percolation [17], critical lattice trees [18], the critical contact process [16], and the voter model [6]; it is believed to be the scaling of critical ordinary percolation in the same regime. The only scaling limit of the above which has been verified at the critical dimension is the voter model [6] where the critical dimension is two. In this case the simple nature of the dual process, a coalescing random walk, allows one to carry out the required explicit calculations. Now our challenge is to use the related but more complex dual of the Barton-Etheridge model to carry through the analysis. It is understood here that we are not taking "long-range" limits (e.g. as was done for the contact process in [11]) which will weaken the interaction and make the analysis considerably easier. In our setting this means not letting the impact factor (described below) approach zero in the rescaling.

We start a rigorous description of the model by recalling the definition of the fixed radius SLFV process given in [5]. Let $r>0$ be the "interaction radius", let $\rho \in[0,1]$ be the "impact factor," and let $\Pi$ be a Poisson point process on $\mathbb{R}^{d} \otimes(0, \infty)$ with intensity $d x \otimes d t$. We suppose the distribution of types in the population changes over time according to "reproduction events" determined by $\Pi$. Given $\mu_{t-}$, if $(x, t) \in \Pi$, choose an independent point $z$ uniformly at random from the Euclidean ball

$$
B_{r}(x)=\{y:|y-x| \leq r\},
$$

and (independently) a type $\alpha$ according to the distribution $\mu_{t-}(z)$, and then set

$$
\mu_{t}(y)=(1-\rho) \mu_{t-}(y)+\rho \delta_{\alpha} \quad \forall y \in B_{r}(x) .
$$

We keep $\mu_{t}(y)=\mu_{t-}(y)$ for $y \notin B_{r}(x)$. Writing $\mu_{t}(x)$ in the form $w_{t}(x) \delta_{1}+\left(1-w_{t}(x)\right) \delta_{0}$, we can reformulate the above dynamics more conveniently in terms of $w_{t}$ as follows. Starting from a Borel $w_{0}: \mathbb{R}^{d} \rightarrow[0,1]$ with compact support, for $(x, t) \in \Pi$, choose an independent parental location $z$ uniformly at random from $B_{r}(x)$, independent of everything, and then:

(i) with probability $w_{t-}(z)$ put $w_{t}(y)=(1-\rho) w_{t-}(y)+\rho$ for all $y \in B_{r}(x)$,

(ii) with probability $1-w_{t-}(z)$ put $w_{t}(y)=(1-\rho) w_{t-}(y)$ for all $y \in B_{r}(x)$,

(iii) for all $y \notin B_{r}(x)$ keep $w_{t}(y)=w_{t-}(y)$.

As noted in Section 3 of [5], this description gives a well-defined $w_{t}: \mathbb{R}^{d} \rightarrow[0,1]$ which has compact support at all times. (See [25] for more details on the construction.) It will be useful to regard $w_{t}$ as the measure $w_{t}(x) d x$, and for bounded Borel $\phi: \mathbb{R}^{d} \rightarrow[0, \infty)$, write

$$
w_{t}(\phi)=\int_{\mathbb{R}^{d}} \phi(x) w_{t}(x) d x .
$$

Closely associated with the process $w_{t}$ is a dual process of coalescing "lineages". If we sample a finite number of spatial locations $\left\{x_{i}\right\}$ at time $T$, it is easy to see that the values $w_{T}\left(x_{i}\right)$ can be determined from $w_{0}$ by using $\Pi$ to trace the lineages backward in time. Since $\Pi$ run backwards is still a Poisson process, we may define a version of the lineages process starting at backwards time 0 from a finite number of locations $\left\{x_{i}\right\}$ as 
follows. If $(x, t) \in \Pi$, mark each lineage in $B_{r}(x)$ independently with probability $\rho$, and choose a point $z$ uniformly at random from $B_{r}(x)$. If at least one of the lineages in $B_{r}(x)$ is marked, all marked lineages in $B_{r}(x)$ coalesce and the resulting lineage is moved to $z$. If no lineage is marked, no lineage moves. Lineages outside of $B_{r}(x)$ are not affected. In this paper it will suffice to consider only the one and two-lineage systems, so we will ignore the higher lineage systems which are more complex to analyze.

We now give a more precise description of these Markov jump processes, using the language of "particles" instead of lineages. Let $|\Gamma|$ be the Lebesgue measure of $\Gamma \subset \mathbb{R}^{d}$. Let $U, U^{1}, U^{1}$ be independent random variables uniformly distributed on $B_{r}=B_{r}(0)$, and let $\bar{U}$ have the law of $U^{1}+U^{2}$, i.e., $\bar{U}$ has density

$$
P(\bar{U} \in d z)=\frac{\left|B_{r}(0) \cap B_{r}(z)\right|}{\left|B_{r}(0)\right|^{2}} d z:=h_{\bar{U}}(z) d z .
$$

We let $\bar{\sigma}^{2} 1_{d \times d}$ denote the covariance matrix of $\bar{U}$, so that if $x=\left(x_{1}, \ldots, x_{d}\right)$, then

$$
\bar{\sigma}^{2}=\frac{2}{\left|B_{r}\right|} \int_{B_{r}}\left(x_{1}\right)^{2} d x .
$$

We will use this notation throughout, along with $\eta_{t}$ for the single particle dual and $\xi_{t}=\left(\xi_{t}^{1}, \xi_{t}^{2}\right)$ for the two particle dual.

(a) The single-particle dual $\eta_{t}$. If we start with a single particle at $x$, it is easy to see that $\eta_{t}$ is the random walk on $\mathbb{R}^{d}$ starting at $x$ which makes jumps at rate $\rho\left|B_{r}\right|$ with jump distribution given in (1.3). We write $P_{\{x\}}$ for the underlying law of $\eta$.

(b) The two-particle dual $\left(\xi_{t}^{1}, \xi_{t}^{2}\right)$. If we start with two particles, one at $x_{1}$ and the other at $x_{2} \neq x_{1},\left(\xi_{t}^{1}, \xi_{t}^{2}\right)$ is the Markov jump process starting at $\left(x_{1}, x_{2}\right)$, and with law $P_{\left\{x_{1}, x_{2}\right\}}$, which makes transitions

$$
\left(y_{1}, y_{2}\right) \rightarrow \begin{cases}(y+\bar{U}, y+\bar{U}) & \text { at rate } \rho\left|B_{r}\right| \text { if } y_{1}=y_{2}=y \\ \left(y_{1}+\bar{U}, y_{2}\right) & \text { at rate } \rho\left(\left|B_{r}\right|-\rho\left|B_{r}\left(y_{1}\right) \cap B_{r}\left(y_{2}\right)\right|\right) \text { if } y_{1} \neq y_{2} \\ \left(y_{1}, y_{2}+\bar{U}\right) & \text { at rate } \rho\left(\left|B_{r}\right|-\rho\left|B_{r}\left(y_{1}\right) \cap B_{r}\left(y_{2}\right)\right|\right) \text { if } y_{1} \neq y_{2} \\ \left(U+U_{y_{1}, y_{2}}, U+U_{y_{1}, y_{2}}\right) & \text { at rate } \rho^{2}\left|B_{r}\left(y_{1}\right) \cap B_{r}\left(y_{2}\right)\right| \text { if } y_{1} \neq y_{2},\end{cases}
$$

where $U_{y_{1}, y_{2}}$ is an independent random variable, uniformly distributed over $B_{r}\left(y_{1}\right) \cap$ $B_{r}\left(y_{2}\right)$. For $y_{1} \neq y_{2}$, the total jump rate at $\left(y_{1}, y_{2}\right), y_{1} \neq y_{2}$, is $2 \rho\left|B_{r}\right|-\rho^{2}\left|B_{r}\left(y_{1}\right) \cap B_{r}\left(y_{2}\right)\right|$. To see the above rates consider, for example, the second transition from $\left(y_{1}, y_{2}\right)$ to $\left(y_{1}+\bar{U}, y_{2}\right)$ for $y_{1} \neq y_{2}$ where $\left(y_{1}, y_{2}\right)$ is the current site of our two-particle dual. The next jump in the first coordinate can only occur at a point $(x, t) \in \Pi$ with $x \in B_{r}\left(y_{1}\right)$ so let $(x, t)$ be the next such point. At $(x, t)$ such a jump (affecting the first coordinate but not the second) can occur in one of two ways: if $x$ lands in $B_{r}\left(y_{1}\right) \backslash\left(B_{r}\left(y_{1}\right) \cap B_{r}\left(y_{2}\right)\right)$ and the particle $\xi^{1}$ at $y_{1}$ is marked, or if $x$ lands in $B_{r}\left(y_{1}\right) \cap B_{r}\left(y_{2}\right)$ and the particle at $y_{1}$ is marked and the particle at $y_{2}$ is not. The total rate in $t$ is obtained by integrating out $x$ and so is

$$
\rho\left(\left|B_{r}\left(y_{1}\right)\right|-\left|B_{r}\left(y_{1}\right) \cap B_{r}\left(y_{2}\right)\right|\right)+\rho(1-\rho)\left|B_{r}\left(y_{1}\right) \cap B_{r}\left(y_{2}\right)\right|=\rho\left|B_{r}\right|-\rho^{2}\left|B_{r}\left(y_{1}\right) \cap B_{r}\left(y_{2}\right)\right| .
$$

In either of the above scenarios the particle at $y_{1}$ will jump to $z$, a uniformly selected site in $B_{r}(x)$. Given $y_{1}, x$ will be uniformly distributed on $B_{r}\left(y_{1}\right)$ and so $x-y_{1}$ will be uniform on $B_{r}$. Clearly given $\left(y_{1}, x\right), z-x$ is uniformly distributed over $B_{r}$ and so $\left(x-y_{1}, z-x\right)$ is a pair of independent uniforms on $B_{r}$. Therefore the jump in $\xi^{1}$ at time $t$ is $z-y_{1}=(z-x)+\left(x-y_{1}\right)$ and so has law $\bar{U}$ as claimed. The other transitions are similar to analyze. 
The coalescence time for the two-particle dual starting at $\left(x_{1}, x_{2}\right)$ is

$$
\tau=\inf \left\{t \geq 0: \xi_{t}^{1}=\xi_{t}^{2}\right\} .
$$

Although $\left(\xi_{t}^{1}, \xi_{t}^{2}\right)$ is Markov, the individual coordinates $\xi_{t}^{1}, \xi_{t}^{2}$ are not (i.e., $\xi^{1}$ is not Markov with respect to the filtration $\sigma\left(\xi_{s}^{1}, 0 \leq s \leq t\right)_{t \geq 0}$ ). However, when $B_{r}\left(\xi_{t}^{1}\right) \cap B_{r}\left(\xi_{t}^{2}\right)=\emptyset$, both coordinates move independently according to the single particle dynamics, while for $t>\tau$, the coalesced coordinates move together according to the single particle dynamics. It is also clear from (1.5) that the two-particle dual is translation invariant, that is,

$$
P_{\left\{x_{1}+x, x_{2}+x\right\}}\left(\left(\xi^{1}, \xi^{2}\right) \in \cdot\right)=P_{\left\{x_{1}, x_{2}\right\}}\left(\left(x+\xi^{1}, x+\xi^{2}\right) \in \cdot\right) \quad \forall x, x_{1}, x_{2} \in \mathbb{R}^{d} .
$$

The two special cases of the general duality equation in Proposition 2.5 of [5] that we need are the following. For all $t \geq 0, \psi_{1} \in C\left(\mathbb{R}^{d}\right) \cap L^{1}\left(\mathbb{R}^{d}\right)$ and $\psi_{2} \in C\left(\mathbb{R}^{d} \times \mathbb{R}^{d}\right) \cap$ $L^{1}\left(\mathbb{R}^{d} \times \mathbb{R}^{d}\right)$,

$$
\begin{aligned}
& E_{w_{0}}\left[w_{t}\left(\psi_{1}\right)\right]=\int_{\mathbb{R}^{d}} \psi_{1}(x) E_{\{x\}}\left[w_{0}\left(\eta_{t}\right)\right] d x \text {, and } \\
& E_{w_{0}}\left[\int_{\mathbb{R}^{d} \times \mathbb{R}^{d}} \psi_{2}\left(x_{1}, x_{2}\right) w_{t}\left(x_{1}\right) w_{t}\left(x_{2}\right) d x_{1} d x_{2}\right] \\
& \quad=\int_{\mathbb{R}^{d} \times \mathbb{R}^{d}} \psi_{2}\left(x_{1}, x_{2}\right) E_{\left\{x_{1}, x_{2}\right\}}\left[w_{0}\left(\xi_{t}^{1}\right) 1_{\{\tau \leq t\}}+w_{0}\left(\xi_{t}^{1}\right) w_{0}\left(\xi_{t}^{2}\right) 1_{\{\tau>t\}}\right] d x_{1} d x_{2} .
\end{aligned}
$$

By standard approximation arguments, these equations then hold for all Borel $\psi_{1}, \psi_{2}$ which are either nonnegative or integrable (on one side or the other). In particular, letting 1 denote the constant function 1 on $\mathbb{R}^{d}$, we have

$$
E_{w_{0}}\left[w_{t}(\mathbf{1})\right]=\int_{\mathbb{R}^{d}} E_{\{x\}}\left[w_{0}\left(\eta_{t}\right)\right] d x=w_{0}(\mathbf{1}) .
$$

Before stating the main fixed radius result of [5], Theorem 2.6, we introduce superBrownian motion using the martingale problem formulation. If $\left(X_{t}\right)_{t \geq 0}$ is a stochastic process, $\left(\mathcal{F}_{t}^{X}\right)_{t \geq 0}$ will denote the right-continuous filtration generated by $X$. Let $\mathcal{M}_{F}\left(\mathbb{R}^{d}\right)$ denote the space of finite Borel measures on $\mathbb{R}^{d}$ endowed with the topology of weak convergence, and for $\mu \in \mathcal{M}_{F}\left(\mathbb{R}^{d}\right)$ let $\mu(\phi)=\int_{\mathbb{R}^{d}} \phi d \mu$. The space of bounded continuous functions on $\mathbb{R}^{d}$ is denoted by $C_{b}\left(\mathbb{R}^{d}\right)$, and $C_{0}^{3}\left(\mathbb{R}^{d}\right)$ is the space of continuous functions on $\mathbb{R}^{d}$ which vanish at infinity and have bounded continuous partials of order 3 and less. Then (see, e.g., Theorem A.1 of [6] for uniqueness, and Theorem II.5.1 and Remark II.5.5 of [22] for existence) Super-Brownian motion with diffusion coefficient $\sigma^{2}$ and branching rate $b$, denoted $\operatorname{SBM}\left(X_{0}, \sigma^{2}, b\right)$, is the unique $\mathcal{M}_{F}\left(\mathbb{R}^{d}\right)$-valued Markov process $\left(X_{t}\right)_{t \geq 0}$ with continuous paths and initial state $X_{0}$, such that such that for every $\phi \in C_{0}^{3}\left(\mathbb{R}^{d}\right)$,

$$
M_{t}(\phi)=X_{t}(\phi)-X_{0}(\phi)-\int_{0}^{t} X_{s}\left(\frac{\sigma^{2}}{2} \Delta \phi\right) d s
$$

is a local $\left(\mathcal{F}_{t}^{X}\right)$-martingale with predictable quadratic variation process

$$
\langle M(\phi)\rangle_{t}=b \int_{0}^{t} X_{s}\left(\phi^{2}\right) d s .
$$

Theorem 2.6 in [5] considers a sequence $X_{t}^{N}=K w_{t}^{N}$ of scaled versions of $w_{t}$, defined with sequences $K=K_{N}, M=M_{N}$, and $J=J_{N}$. Namely, given $w_{0}^{N}, w_{t}^{N}$ is constructed in the same way as $w_{t}$, but with the following modifications. If $(x, t) \in \Pi$ then there is a reproduction event at $\left(\frac{x}{M}, \frac{t}{N}\right)$, with impact factor $\frac{\rho}{J}$ and reproduction region $B_{\frac{r}{M}}\left(\frac{x}{M}\right)$. 
Thus, for $w_{t}^{N}$, time is sped up by $N$, space is shrunk by $M_{N}$, and the impact factor is reduced by $J_{N} \geq 1$. Denote the rescaled Poisson point process with intensity $M^{d} N d x \otimes d t$ by

$$
\Pi^{N}=\left\{\left(\frac{x}{M}, \frac{t}{N}\right):(x, t) \in \Pi\right\} .
$$

If $n_{t}^{N}(A)=\#\left\{(s, x) \in \Pi^{N} \cap([0, t] \times A)\right\}$, let $\left(\mathcal{F}_{t}^{N}\right)$ be the right-continuous filtration generated by $\left\{n_{s}^{N}(A): s \leq t, A\right.$ a Borel set in $\left.\mathbb{R}^{d}\right\}$.

Let $D\left([0, \infty), \mathcal{M}_{F}\left(\mathbb{R}^{d}\right)\right)$ denote the space of cadlag $\mathcal{M}_{F}\left(\mathbb{R}^{d}\right)$-valued paths equipped with the Skorokhod (J1) topology.

Theorem 1.0 (Theorem 2.6 in [5]). Suppose that for a compact set $D_{0} \subset \mathbb{R}^{d}$, $\operatorname{supp}\left(w_{0}^{N}\right) \subset$ $D_{0}$ for all $N$, and as elements of $\mathcal{M}_{F}\left(\mathbb{R}^{d}\right), X_{0}^{N} \rightarrow X_{0} \in \mathcal{M}_{F}\left(\mathbb{R}^{d}\right)$ as $N \rightarrow \infty$. In addition, suppose there are constants $C_{1}, C_{2} \in(0, \infty)$ such that, as $N \rightarrow \infty$,

(1) $M \rightarrow \infty$,

(2) $\frac{N}{J M^{2}} \rightarrow C_{1}$,

(3) $\frac{K N}{J^{2} M^{d}} \rightarrow C_{2}$,

(4) $\begin{cases}\frac{M}{J} \rightarrow 0 & \text { if } d=1, \\ \frac{\log M}{J} \rightarrow 0 & \text { if } d=2, \\ \frac{1}{J} \rightarrow 0 & \text { if } d \geq 3 .\end{cases}$

Then the sequence $\left(X^{N}\right)_{N \geq 1}$ converges weakly in $D\left([0, \infty), \mathcal{M}_{F}\left(\mathbb{R}^{d}\right)\right)$ to $\operatorname{SBM}\left(X_{0}, \sigma^{2}, b\right)$ with

$$
\sigma^{2}=2 C_{1} \rho \int_{B_{r}}\left(x_{1}\right)^{2} d x \text { and } b=C_{2} \rho^{2}\left|B_{r}\right|^{2} .
$$

(The constant $C(d)$ in Definition 4.1 in [5] should be $C(d)=\int_{B_{1}}\left(x_{1}\right)^{2} d x$.) As noted in [5], this result is similar in spirit to Theorem 1.1 in [6], which proves convergence to SBM for certain sparse "long range" kernel voter models. Due to conditions (1) and (4) above, $J_{N} \rightarrow \infty$ and hence the impact factors $\rho / J_{N} \rightarrow 0$. It is this fact and the mass scaling condition (3) which make these SLFV processes analogous to the long range voter models in [6]. As for the duals, conditions (1) and (2) ensure that the single particle dual motion converges to Brownian motion, while the condition $J_{N} \rightarrow \infty$ ensures that the interactions between dual particles are weak.

If the sequence $J_{N}$ were bounded, so that the impact factors $\rho=\rho / J_{N}$ do not vanish in the limit, the resulting SLFV processes would correspond to the "fixed" kernel voter models in Theorem 1.2 in [6]. In biological terms this corresponds to keeping the "neighbourhood size" finite in the scaling limit, while letting $J_{N} \rightarrow \infty$ effectively allows this parameter to become infinite; see the discussion in Section 2 of [14] and especially Definition 2.2 there. In that work they showed in this fixed neighbourhood size setting (Theorem 2.7 of [14]) that, with an appropriate selection term, the dual particle process converges to a branching Brownian motion in the scaling limit. The purpose of this paper is to prove that in this setting, with no selection, there is also a forwards limit theorem giving convergence to SBM.

Throughout this work we will assume $d \geq 2$, and

$$
N \geq 3 \text {. }
$$

For our scaled SLFV processes $w_{t}^{N}$ we then make the choices

$$
J=1, \quad M=\sqrt{N}, \quad K= \begin{cases}N^{\frac{d}{2}-1} & \text { for } d \geq 3 \\ \log N & \text { for } d=2 .\end{cases}
$$


If we set $J \equiv 1$, and take $C_{1}=C_{2}=1$ for simplicity, the conditions (1)-(3) in Theorem 1.0 suggest the choices for $M$ and $K$ above except for the logarithmic correction to $K$ for $d=2$. Without this correction, one can show that the limiting process in Theorem 1.2 would be nonrandom heat flow acting on $X_{0}$, as is the case for the voter model [23]. We do not consider the case $d=1$ in (1.14). For this case, the Wright-Fisher SPDE was obtained in [15] as an appropriate scaling limit of SLFV, but under that assumption that the scaled impact factors approach zero like $N^{-1 / 3}$ (see [21] for the corresponding scaling limit for the voter model). If the impact factors were bounded away from zero, the strong recurrence of one-dimensional random walk would lead to heavy clustering, resulting in scaling limits with segregation of types; the corresponding scaling limit for the voter model is the Arratia flow [1], not super-Brownian motion.

In order to state our limit theorem for scaled SLFV processes assuming (1.14), we must first identify certain constants $\gamma_{e}^{(d)}$ that appear in the limiting SBM branching rate. These constants are determined by the asymptotic tail behavior of the coalescence times $\tau$ for the unscaled two-particle dual process defined in (1.5). Introduce

$$
\gamma_{e}(t)=\gamma_{e}^{(d)}(t)=\frac{1}{\left|B_{r}\right|^{2}} \int_{B_{r}} \int_{B_{r}} P_{\left\{x_{1}, x_{2}\right\}}(\tau>t) d x_{1} d x_{2} .
$$

Proposition 1.1. There are constants $\gamma_{e}=\gamma_{e}^{(d)}>0$ such that as $t \rightarrow \infty$,

$$
\lim _{t \rightarrow \infty} \gamma_{e}^{(d)}(t)=\gamma_{e} \text { if } d \geq 3
$$

and

$$
\lim _{t \rightarrow \infty}(\log t) \gamma_{e}^{(d)}(t)=\gamma_{e} \text { if } d=2
$$

Recall that when outside $B_{2 r}, \xi_{t}^{1}-\xi_{t}^{2}$ behaves like a rate $2 \rho\left|B_{r}\right|$ random walk with jump distribution given in (1.3), and $\tau=\inf \left\{t \geq 0: \xi_{t}^{1}-\xi_{t}^{2}=0\right\}$. Therefore, if $d \geq 3$, the difference will escape to infinity with positive probability by transience, and so the limit in (1.15), which exists by monotonicity, will have a non-zero limit. For $d=2$ the situation is more delicate. One can predict the $1 / \log t$ behaviour of $\gamma_{e}(t)$ from the corresponding non-return probabilities for irreducible symmetric random walk on $\mathbb{Z}^{2}$ with diagonal covariance matrix (see, e.g., Lemma A.3(ii) of [6]), but the slowing rates when the difference $\xi^{1}-\xi^{2}$ is in $B_{2 r}$ complicates things. The limit (1.16) can be derived from Lemma 4.10 in [14]. The analysis there is based on a construction using successive "inner" and "outer" excursions of $\xi^{1}-\xi^{2}$ from certain balls before coalescence occurs. Our argument represents the difference process as a time change of a rate $2 \rho\left|B_{r}\right|$ random walk with step distribution $h_{\bar{U}}$, and makes use of a reflection coupling. We feel the proof is of independent interest and so have included it in an Appendix. One advantage of the excursion approach in [14] is that it should also allow inclusion of a random "interaction radius", that is

the driving Poisson point process $\Pi$ is now on $\mathbb{R}^{d} \times[0, \infty) \times\left[0, r_{\max }\right]$ with intensity

$d x \otimes d t \otimes \mu(d r)$ for some finite measure $\mu$ on the compact interval $\left[0, r_{\max }\right]$.

However, as is discussed below, our time-change representation of the dual difference process in the fixed radius case will also play an important role in the analysis of the martingale square function which is the key ingredient in the proof of our main convergence result, Theorem 1.2 below.

With the choice of renormalization constants in (1.14) we now give a different description of the rescaled SLFV processes $X^{N}$, which will clarify the comparison with 
Theorem 1.2 below of the fixed kernel voter model result in [6]. Assume $X_{0} \in \mathcal{M}_{F}\left(\mathbb{R}^{d}\right)$ and the compactly supported initial conditions $\bar{w}_{0}^{N}: \mathbb{R}^{d} \rightarrow[0,1]$ satisfy

$$
K_{N} \bar{w}_{0}^{N}(\sqrt{N} x) d x \rightarrow X_{0} \text { in } \mathcal{M}_{F}\left(\mathbb{R}^{d}\right) .
$$

For each $N$, let $\bar{w}^{N}$ be the (original, unscaled) SLFV process defined in (1.1) with fixed interaction radius $r$, fixed impact factor $\rho$ and initial condition $w_{0}^{N}=\bar{w}_{0}^{N}$, and define the rescaled SLFV process by

$$
w_{t}^{N}(x)=\bar{w}_{N t}^{N}(\sqrt{N} x)
$$

This process has the same law as $w^{N}$ defined using $\Pi^{N}$ right before Theorem 1.0, with $J$ and $M$ given in (1.14). (For example, if $(x, s)$ is the first point in $\Pi$ affecting $\bar{w}^{N}$, then $\bar{w}_{s}^{N} \neq \bar{w}_{s-}^{N}$ is only possible inside $B_{r}(x)$. Letting $N t=s$, this means that $w_{t}^{N}(y) \neq w_{t-}^{N}(y)$ is possible only for $y \sqrt{N} \in B_{r}(x)$, or $y \in B_{r / \sqrt{N}}(x)$. Thus the interaction radius for $w^{N}$ is $r / \sqrt{N}$.) Finally our approximating empirical measures are given by

$$
X_{t}^{N}(d x)=K_{N} w_{t}^{N}(x) d x:=X_{t}^{N}(x) d x \in \mathcal{M}_{F}\left(\mathbb{R}^{d}\right),
$$

so that (1.18) just asserts that $X_{0}^{N} \rightarrow X_{0}$. A simple change of variables shows that in terms of the unscaled SLFV processes, $\bar{w}^{N}$, we have for any bounded Borel $\phi$ on $\mathbb{R}^{d}$,

$$
X_{t}^{N}(\phi)= \begin{cases}\frac{1}{N} \int_{\mathbb{R}^{d}} \bar{w}_{N t}^{N}(y) \phi\left(y N^{-1 / 2}\right) d y & \text { if } d \geq 3, \\ \frac{\log N}{N} \int_{\mathbb{R}^{d}} \bar{w}_{N t}^{N}(y) \phi\left(y N^{-1 / 2}\right) d y & \text { if } d=2 .\end{cases}
$$

Here is our main result for the scaled SLFV process. For a measure or function $H$, we let $\operatorname{supp}(H)$ denote its closed support. Recall the definition of $\bar{\sigma}^{2}$ from (1.4).

Theorem 1.2. Suppose that $d \geq 2, \operatorname{supp}\left(w_{0}^{N}\right)$ is compact for each $N$, and $\lim _{N \rightarrow \infty} X_{0}^{N}=$ $X_{0}$ in $\mathcal{M}_{F}\left(\mathbb{R}^{d}\right)$. As $N \rightarrow \infty, X^{N}$ converges weakly in $D\left([0, \infty), \mathcal{M}_{F}\left(\mathbb{R}^{d}\right)\right)$ to $\operatorname{SBM}\left(X_{0}, \sigma^{2}, b\right)$, where

$$
\sigma^{2}=\rho\left|B_{r}\right| \bar{\sigma}^{2} \text { and } b=\rho^{2}\left|B_{r}\right|^{2} \gamma_{e} .
$$

As is usual for SBM limit theorems, the scaling condition on the initial conditions in (1.18) corresponds to a regime where type 1's are scarce. For example, if, say, $X_{0}$ assigns no mass to the boundary of $[-1,1]^{d}$, then taking $\phi=1\left(\|x\|_{\infty} \leq 1\right)$ we have $X_{0}^{N}(\phi) \rightarrow X_{0}\left([-1,1]^{d}\right)$ which implies

$$
\frac{\int_{[-\sqrt{N}, \sqrt{N}]^{d}} \bar{w}_{0}^{N}(x) d x}{(2 \sqrt{N})^{d}} \sim \frac{X_{0}\left([-1,1]^{d}\right)}{2^{d / 2} K_{N}} \rightarrow 0 \text { as } N \rightarrow \infty .
$$

It is important to note that in our scaling regime with $J=1$, the original $\bar{w}^{N}$ we are working with is an ordinary SLVF process with fixed interaction range $r$ and impact factor $\rho$, but with an initial condition in which type 1's are scarce.

Equation (1.21) should be compared to the corresponding rescaled empirical measures in [6] associated with a sequence of voter models $\xi_{t}^{(N)}(x), x \in \mathbb{Z}^{d}$ whose rescaled initial states again converge to a limiting $X_{0} \in \mathcal{M}_{F}\left(\mathbb{R}^{d}\right)$ : if $N^{\prime}=N$ for $d \geq 3$ and $N^{\prime}=N / \log N$ if $d=2$, let

$$
\hat{X}_{t}^{N}=\frac{1}{N^{\prime}} \sum_{x \in \mathbb{Z}^{d}} \xi_{N t}^{(N)}(x) \delta_{x N^{-1 / 2}}
$$

In that reference it is shown that $\hat{X}^{N}$ converges weakly in $D\left([0, \infty), \mathcal{M}_{F}\left(\mathbb{R}^{d}\right)\right)$ to an appropriate SBM, whose branching rate is determined by the asymptotics of the escape probability (from 0) for a continuous time random walk starting at a uniformly chosen neighbour of 0 in the integer lattice through a two-particle dual calculation. This suggests 
the same should hold (as it does) for the SLFV but now with the asymptotics of the non-coalescing probability of our two particle dual playing the role of the random walk escape probability.

The proof follows a familiar outline, based in part on methods in [6]. For appropriate test functions $\phi$ the semimartingale decomposition from [5], recalled in Section 2, states that

$$
X_{t}^{N}(\phi)=X_{0}^{N}(\phi)+D_{t}^{N}(\phi)+M_{t}^{N}(\phi),
$$

where $M^{N}(\phi)$ is a local martingale, and $D^{N}(\phi)$ is a drift term of bounded variation. In Section 2 we provide some elementary simplifications for the explicit expressions for both $D^{N}(\phi)$ and the predictable quadratic variation process $\left\langle M^{N}(\phi)\right\rangle_{t}$ from [5]. In Section 3 we use the above and the one- and two-particle duals to calculate the first moments of $X_{t}^{N}$ and give uniform $L^{2}$ bounds on the total mass $X_{t}^{N}(\mathbf{1})$ (Corollary 3.2) which will be used throughout.

Assuming the key Proposition 4.1 which is proved in Section 7, tightness of $\left\{X^{N}\right\}$ is then established in Section 4, where Theorem 1.2 is also proved by showing that any weak limit satisfies the martingale problem for $\operatorname{SBM}\left(X_{0}, \sigma^{2}, b\right)$. The term $D^{N}(\phi)$ is easy to handle (Lemma 2.3); it is the asymptotic behavior of the quadratic variation process $\left\langle M^{N}(\phi)\right\rangle$ which requires some work. The key result here is the aforementioned Proposition 4.1 which we present here for the discussion below.

Proposition (4.1). For all $A, T>0$, and $\phi \in C_{0}^{3}$,

$$
\sup _{X_{0}^{N}(\mathbf{1}) \leq A} E\left[\left.\sup _{0 \leq t \leq T}\left|\left\langle M^{N}(\phi)\right\rangle_{t}-\int_{0}^{t} \rho^{2}\right| B_{r}\right|^{2} \gamma_{e} X_{s}^{N}\left(\phi^{2}\right) d s \mid\right] \rightarrow 0 \text { as } N \rightarrow \infty .
$$

After establishing preliminary random walk results in Section 5 and facts about twoparticle duals in Section 6, it is proved in Section 7. Its proof uses Proposition 1.1 but the issues go well beyond this result.

The behavior of the quadratic variation process is the main difference in the proofs of Theorem 1.2 and its counterpart in [5], Theorem 1.0. Lemma 4.3 in [5] shows that a key term in the variation process is negligible in the limit $N \rightarrow \infty$. This fact is a consequence of the assumption $J \rightarrow \infty$. In our case, with $J \equiv 1$, this term is nonnegligible, and in fact determines the limiting SBM branching rate. Its analysis is the main objective of Section 7. The analysis for $d \geq 3$ is straightforward; it is the 2-dimensional case (the most relevant from a biological perspective) that is the most interesting. In this setting the proof requires an extension of the arguments in [6] and [8] used to analyze the voter model and stochastic Lotka-Volterra models, respectively. The analogues of Proposition 4.1 in [6] ((I1) in that reference) and [8] (Proposition 4.7 in this work) involved $L^{2}$ and $L^{p}$ ( $p>1$ is used) norms, respectively, instead of the $L^{1}$ norm in Proposition 4.1, but also had no supremum over time in the expectation. When the $L^{2}$ norm is expanded in the voter model paper this leads to a four-particle dual calculation, while for the more general stochastic Lotka-Volterra models considered in [8], a trick using the Markov property reduced this to a three-particle dual calculation. Here, because of the non-Markovian property of individual coordinates in the dual, similar calculations seem out of reach and we are led to the $L^{1}$ convergence in Proposition 4.1 which must be established using only one- and two-particle duals. The first issue here is that squares are easier to handle than absolute values (the $p>1$ in [8] is bounded eventually by a square using a stopping argument), and here the innocuous looking Lemma 7.9 below allows one to handle the square (even with a supremum over time) by using a martingale argument. This then enables us to take absolute values inside the time integral where two-particle duals (albeit more complicated ones than those in [8]) can handle the calculation. Here a second issue arises as even in handling a second 
moment calculation in Proposition 7.2 of [8], the use of stochastic calculus there leads to a three-particle calculation. We follow a more efficient path in its analogue, Lemma 7.8 , in Section 7 which only involves the two-particle dual. A third issue is the fact that the weaker $L^{1}$ convergence in Proposition 4.1 will require some additional technical work to establish the local uniform integrability of the $\left\{M^{N}(\phi)_{t}^{2}: N\right\}$, and hence identify the limiting square function. This is what occupies most of the proof of Theorem 1.2 in Section 4.

As a small bonus, the fact that Proposition 4.1 controls the square functions uniformly in time means it also allows one to establish tightness without any higher moments. The required properties of the two-particle dual are established in Section 6. Lemma 6.1 represents the difference of the coordinates of the dual as the time change of a continuous time random walk and this result is then used to obtain several probability estimates on the two-particle dual. These results (notably Lemmas 6.3 to 6.7) then play a central role in Section 7. The time-change is particularly useful when controlling the two-particle dual when the particles are close together and the dual motions slow down.

It would be interesting to see if it is possible to extend Theorem 1.2 to the variable but bounded radius case discussed above.

Constants. In proofs, $C$ will denote a positive constant whose value may change from line to line. We will use $C_{T}$ and $C_{\phi}$ for constants depending on $T>0$ or functions $\phi$ in a similar way. In some cases constants will be numbered and dependence on various quantities indicated explicitly. Finally, most constants will have an implicit dependence on the impact radius $r$, this dependence will be pointed out in some cases for clarity.

\section{Semimartingale characterization of the SLFV}

Let $B_{r}^{N}(x)=B_{r / \sqrt{N}}(x), B_{r}^{N}=B_{r}^{N}(0)$. For $d \geq 2$ and $\phi \in C_{b}\left(\mathbb{R}^{d}\right)$, let

$$
\begin{aligned}
d_{s}^{N}(\phi)= & \frac{\rho N^{1+d}}{\left|B_{r}\right|} \int_{\mathbb{R}^{d}} \int_{B_{r}^{N}(x)}\left\{X_{s}^{N}(z) \int_{B_{r}^{N}(x)} \phi(y) d y-\int_{B_{r}^{N}(x)} \phi(y) X_{s}^{N}(y) d y\right\} d z d x, \\
m_{s}^{N}(\phi)= & \frac{\rho^{2} N^{1+d}}{\left|B_{r}\right|} \int_{\mathbb{R}^{d}} \int_{B_{r}^{N}(x)}\left\{\left(1-\frac{X_{s}^{N}(z)}{K}\right)\left(\int_{B_{r}^{N}(x)} \phi(y) X_{s}^{N}(y) d y\right)^{2}\right. \\
& \left.+\frac{X_{s}^{N}(z)}{K}\left(K \int_{B_{r}^{N}(x)} \phi(y) d y-\int_{B_{r}^{N}(x)} \phi(y) X_{s}^{N}(y) d y\right)^{2}\right\} d z d x .
\end{aligned}
$$

Lemma 2.1. Let $\phi \in C_{b}\left(\mathbb{R}^{d}\right)$ and $\Delta X_{s}^{N}(\phi)=\left|X_{s}^{N}(\phi)-X_{s-}^{N}(\phi)\right|$. Then with probability one,

$$
\left|\Delta X_{s}^{N}(\phi)\right| \leq\|\phi\|_{\infty}\left\{\begin{array}{ll}
\rho\left|B_{r}\right| / N & \text { if } d \geq 3 \\
\rho\left|B_{r}\right| \log N / N & \text { if } d=2
\end{array} \quad \text { for all } s \geq 0\right.
$$

Proof. By the dynamics (1.1), for $(x, s) \in \Pi^{N}$ (we may assume there is at most one such $x), w_{s}^{N}(y)=w_{s-}^{N}(y)$ for all $y \notin B_{r}^{N}(x)$, and for $y \in B_{r}^{N}(x)$,

$$
w_{s}^{N}(y)-w_{s-}^{N}(y)=\left\{\begin{array}{l}
-\rho w_{s-}^{N}(y)+\rho, \text { or } \\
-\rho w_{s-}^{N}(y)
\end{array}\right.
$$

Thus, $\left|w_{s}^{N}(y)-w_{s-}^{N}(y)\right| \leq \rho 1_{B_{r}^{N}(x)}(y)$, and so,

$$
\int_{\mathbb{R}^{d}}\left|w_{s}^{N}(y)-w_{s-}^{N}(y)\right| d y \leq \sup _{x \in \mathbb{R}^{d}} \int_{B_{r}^{N}(x)} \rho d y \leq \rho\left|B_{r}^{N}\right| .
$$


Finally,

$$
\left|\Delta X_{s}^{N}(\phi)\right| \leq K \int_{\mathbb{R}^{d}}\left|\phi ( y ) \left\|w_{s}^{N}(y)-w_{s-}^{N}(y)\left|d y \leq\|\phi\|_{\infty} \rho\right| B_{r} \mid K N^{-d / 2},\right.\right.
$$

which is (2.3).

The martingale characterization below is provided by Lemma 3.1 of [5]. The filtration below is implicit in their argument. Although $\phi=1$ is not included in that result it is easy to handle it by a localization argument using the stopping times $T_{n}=\inf \{t \geq 0$ : $\left.\int_{\{|x| \geq n\}} w_{t}(x) d x>0\right\}$. Recall $\mathcal{F}_{t}^{N}$ is defined prior to Theorem 1.0.

Proposition 2.2. Let $\phi \in C_{0}^{3}\left(\mathbb{R}^{d}\right)$ or $\phi=1$ Then $X_{t}^{N}(\phi)$ has the semimartingale decomposition:

$$
X_{t}^{N}(\phi)=X_{0}^{N}(\phi)+D_{t}^{N}(\phi)+M_{t}^{N}(\phi)
$$

where

$$
D_{s}^{N}(\phi)=\int_{0}^{t} d_{s}^{N}(\phi) d s
$$

and $M_{t}^{N}(\phi)$ is a local $\left(\mathcal{F}_{t}^{N}\right)$-martingale with predictable quadratic variation

$$
\left\langle M^{N}(\phi)\right\rangle_{t}=\int_{0}^{t} m_{s}^{N}(\phi) d s .
$$

Implicit in the above is the fact that the local martingale $M_{t}^{N}(\phi)$ is locally square integrable, but this is already clear from the fact that it has bounded jumps. The latter follows from Lemma 2.1 and (2.4) which imply

$$
\left|\Delta M_{s}^{N}(\phi)\right| \leq\|\phi\|_{\infty}\left\{\begin{array}{ll}
\rho\left|B_{r}\right| / N & \text { if } d \geq 3 \\
\rho\left|B_{r}\right| \log N / N & \text { if } d=2
\end{array} \quad \text { for all } s \geq 0 .\right.
$$

For the drift term $D_{s}^{N}(\phi)$ we will need only the following facts.

\section{Lemma 2.3.}

(a) $d_{s}^{N}(\mathbf{1}) \equiv 0$.

(b) For $\phi \in C_{0}^{3}\left(\mathbb{R}^{d}\right)$ there is a constant $C_{2.8}=C_{2.8}(\phi)>0$ such that

$$
\begin{aligned}
d_{s}^{N}(\phi) & =\rho\left|B_{r}\right| \bar{\sigma}^{2} X_{s}^{N}\left(\frac{1}{2} \Delta \phi\right)+\mathcal{E}_{2.8}^{N}(s), \text { where } \\
\left|\mathcal{E}_{2.8}^{N}(s)\right| & \leq C_{2.8} \frac{X_{s}^{N}(\mathbf{1})}{\sqrt{N}} .
\end{aligned}
$$

Part (a) follows easily from (2.1), and (b) is the special case of Lemma 4.2 (and its proof) in [5] for our choices of $J, M, K$ in (1.14). (We note that the constant $C(d)$ in Definition 4.1 in [5] is $\int_{B_{1}}\left(x_{1}\right)^{2} d x$.) Turning next to the martingale square function, for $\phi \in C_{b}\left(\mathbb{R}^{d}\right)$ define

$$
\bar{m}_{s}^{N}(\phi)=\rho^{2} N^{1+d / 2} K^{2} \int_{\mathbb{R}^{d}} \phi^{2}(x) \int_{B_{r}^{N}(x)} \int_{B_{r}^{N}(x)}\left(1-w_{s}^{N}\left(z_{1}\right)\right) w_{s}^{N}\left(z_{2}\right) d z_{2} d z_{1} d x \geq 0 .
$$

\section{Lemma 2.4}

(a) For $\phi \in C_{0}^{3}\left(\mathbb{R}^{d}\right), \bar{m}_{s}^{N}(\phi) \vee m_{s}^{N}(\phi) \leq\|\phi\|_{\infty}^{2} m_{s}^{N}(\mathbf{1})$.

(b) $m_{s}^{N}(\mathbf{1})=\bar{m}_{s}^{N}(\mathbf{1})$.

(c) For $\phi \in C_{0}^{3}\left(\mathbb{R}^{d}\right)$ there is a constant $C_{2.10}=C_{2.10}(\phi)>0$ such

$$
\begin{gathered}
m_{s}^{N}(\phi)=\bar{m}_{s}^{N}(\phi)+\mathcal{E}_{2.10}^{N}(s), \text { where } \\
\left|\mathcal{E}_{2.10}^{N}(s)\right| \leq C_{2.10} \begin{cases}\frac{1}{\sqrt{N}} X_{s}^{N}(\mathbf{1}) & \text { if } d \geq 3 \\
\frac{\log N}{\sqrt{N}} X_{s}^{N}(\mathbf{1}) & \text { if } d=2 .\end{cases}
\end{gathered}
$$


Proof. Define

$$
\mathcal{I}\left(z_{1}, z_{2}, z_{3}\right)=\left(1-w_{s}^{N}\left(z_{1}\right)\right) w_{s}^{N}\left(z_{2}\right) w_{s}^{N}\left(z_{3}\right)+w_{s}^{N}\left(z_{1}\right)\left(1-w_{s}^{N}\left(z_{2}\right)\right)\left(1-w_{s}^{N}\left(z_{3}\right)\right) .
$$

Then replacing $X_{s}^{N}$ with $K w_{s}^{N}$ in (2.2), after expanding and rearranging, we find that

$$
m_{s}^{N}(\phi)=\frac{\rho^{2} N^{1+d}}{\left|B_{r}\right|} K^{2} \int_{\mathbb{R}^{d}} \int_{B_{r}^{N}(x)} \int_{B_{r}^{N}(x)} \int_{B_{r}^{N}(x)} \phi\left(z_{2}\right) \phi\left(z_{3}\right) \mathcal{I}\left(z_{1}, z_{2}, z_{3}\right) d z_{3} d z_{2} d z_{1} d x
$$

On account of $0 \leq w_{s}^{N} \leq 1, \mathcal{I}$ is nonnegative, hence (a) follows for $m^{N}$ from the above expression, and is immediate for $\bar{m}^{n}$ from (2.9) (integrate out $z_{3}$ in the first line on the right-hand side). If we define $\Delta_{\phi}\left(x, z_{2}, z_{3}\right)=\phi\left(z_{2}\right) \phi\left(z_{3}\right)-\phi^{2}(x)$, then

$$
m_{s}^{N}(\phi)=\frac{\rho^{2} N^{1+d}}{\left|B_{r}\right|} K^{2} \int_{\mathbb{R}^{d}} \int_{B_{r}^{N}(x)} \int_{B_{r}^{N}(x)} \int_{B_{r}^{N}(x)} \phi^{2}(x) \mathcal{I}\left(z_{1}, z_{2}, z_{3}\right) d z_{3} d z_{2} d z_{1} d x+\mathcal{E}_{2.10}^{N}(s),
$$

where

$$
\mathcal{E}_{2.10}^{N}(s)=\rho^{2} \frac{N^{1+d}}{\left|B_{r}\right|} K^{2} \int_{\mathbb{R}^{d}} \int_{B_{r}^{N}(x)} \int_{B_{r}^{N}(x)} \int_{B_{r}^{N}(x)} \Delta_{\phi}\left(x, z_{2}, z_{3}\right) \mathcal{I}\left(z_{1}, z_{2}, z_{3}\right) d z_{3} d z_{2} d z_{1} d x
$$

Consider the integrals over $B_{r}^{N}(x)$ in (2.11). By a change of variables and order of integration,

$$
\begin{aligned}
\int_{B_{r}^{N}(x)} \int_{B_{r}^{N}(x)} & \int_{B_{r}^{N}(x)} \mathcal{I}\left(z_{1}, z_{2}, z_{3}\right) d z_{3} d z_{2} d z_{1} \\
= & \int_{B_{r}^{N}(x)} w_{s}^{N}\left(z_{3}\right) \int_{B_{r}^{N}(x)} \int_{B_{r}^{N}(x)}\left(1-w_{s}^{N}\left(z_{1}\right)\right) w_{s}^{N}\left(z_{2}\right) d z_{2} d z_{1} d z_{3} \\
& \quad+\int_{B_{r}^{N}(x)}\left(1-w_{s}^{N}\left(z_{3}\right)\right) \int_{B_{r}^{N}(x)} \int_{B_{r}^{N}(x)}\left(1-w_{s}^{N}\left(z_{1}\right)\right) w_{s}^{N}\left(z_{2}\right) d z_{2} d z_{1} d z_{3} \\
= & \left|B_{r}^{N}\right| \int_{B_{r}^{N}(x)} \int_{B_{r}^{N}(x)}\left(1-w_{s}^{N}\left(z_{1}\right)\right) w_{s}^{N}\left(z_{2}\right) d z_{2} d z_{1} .
\end{aligned}
$$

Plugging this into (2.11), and using the definition of $\bar{m}_{s}^{N}(\phi)$, we now have $m_{s}^{N}(\phi)=$ $\bar{m}_{s}^{N}(\phi)+\mathcal{E}_{2.10}^{N}(s)$.

In the case that $\phi=\mathbf{1}, \Delta_{\mathbf{1}} \equiv 0$ so that $\mathcal{E}_{2.10}^{N}(s) \equiv 0$, proving (b). More generally,

$$
\left|\Delta_{\phi}\left(x, z_{2}, z_{3}\right)\right| \leq 2(r / \sqrt{N})\|\phi\|_{\infty}\|\phi\|_{\text {Lip }} \text { for } z_{2}, z_{3} \in B_{r}^{N}(x) .
$$

Using the fact that $\left|\mathcal{I}\left(z_{1}, z_{2}, z_{3}\right)\right| \leq w_{s}^{N}\left(z_{2}\right)+w_{s}^{N}\left(z_{1}\right)$, we have

$$
\begin{aligned}
\left|\mathcal{E}_{2.10}^{N}(s)\right| \leq \rho^{2} \frac{N^{1+d}}{\left|B_{r}\right|} K^{2} 2(r / \sqrt{N})\|\phi\|_{\infty}\|\phi\|_{\text {Lip }} \int_{\mathbb{R}^{d}} \int_{B_{r}^{N}(x)} \int_{B_{r}^{N}(x)} \int_{B_{r}^{N}(x)}\left(w_{s}^{N}\left(z_{1}\right)\right. & \left.+w_{s}^{N}\left(z_{2}\right)\right) d z_{2} d z_{1} d z_{3} d x \\
= & 4 \rho^{2} \frac{r}{N^{1 / 2}}\|\phi\|_{\infty}\|\phi\|_{\operatorname{Lip}} \frac{N^{1+d}}{\left|B_{r}\right|}\left|B_{r}^{N}\right|^{2} K^{2} \int_{\mathbb{R}^{d}} \int_{B_{r}^{N}(x)} w_{s}^{N}\left(z_{1}\right) d z_{1} d x \\
& =4 r \rho^{2}\left|B_{r}\right|^{2}\|\phi\|_{\infty}\|\phi\|_{\operatorname{Lip}} \begin{cases}\frac{1}{\sqrt{N}} X_{s}^{N}(\mathbf{1}) & \text { if } d \geq 3 \\
\frac{\log N}{\sqrt{N}} X_{s}^{N}(\mathbf{1}) & \text { if } d=2 .\end{cases}
\end{aligned}
$$

This proves (c). 


\section{Total mass bounds}

We start with the dual particle systems for the rescaled SLFV process $w_{t}^{N}$ in (1.19). If $\eta$ and $\left(\xi^{1}, \xi^{2}\right)$ are as in (1.8), (1.9), introduce the rescaled duals,

$$
\begin{gathered}
P_{\{x\}}^{N}\left(\eta_{t}^{N} \in \cdot\right)=P_{\{\sqrt{N} x\}}\left(\frac{\eta_{N t}}{\sqrt{N}} \in \cdot\right), \quad x \in \mathbb{R}^{d}, \\
P_{\left\{x_{1}, x_{2}\right\}}^{N}\left(\left(\xi_{t}^{N, 1}, \xi_{t}^{N, 2}\right) \in \cdot\right)=P_{\left\{\sqrt{N} x_{1}, \sqrt{N} x_{2}\right\}}\left(\left(\frac{\xi_{N t}^{1}}{\sqrt{N}}, \frac{\xi_{N t}^{2}}{\sqrt{N}}\right) \in \cdot\right) \quad x_{i} \in \mathbb{R}^{d},
\end{gathered}
$$

and

$$
\tau^{N}=\inf \left\{t: \xi_{t}^{N, 1}=\xi_{t}^{N, 2}\right\}
$$

Then (1.8) and (1.9) imply for Borel $\psi_{1}$ on $\mathbb{R}^{d}$, and Borel $\psi_{2}$ on $\left(\mathbb{R}^{d}\right)^{2}$, and $t \geq 0$ (recall $(1.2))$,

$$
\begin{aligned}
& E_{w_{0}^{N}}\left[w_{t}^{N}\left(\psi_{1}\right)\right]=\int_{\mathbb{R}^{d}} \psi_{1}(x) E_{\{x\}}^{N}\left(w_{0}^{N}\left(\eta_{t}^{N}\right)\right) d x \\
& E_{w_{0}^{N}}\left[\int_{\mathbb{R}^{d} \times \mathbb{R}^{d}} \psi_{2}\left(x_{1}, x_{2}\right) w_{t}^{N}\left(x_{1}\right) w_{t}^{N}\left(x_{2}\right) d x_{1} d x_{2}\right] \\
& \quad=\int_{\mathbb{R}^{d} \times \mathbb{R}^{d}} \psi_{2}\left(x_{1}, x_{2}\right) E_{\left\{x_{1}, x_{2}\right\}}^{N}\left[w_{0}^{N}\left(\xi_{t}^{N, 1}\right) 1_{\left\{\tau^{N} \leq t\right\}}+w_{0}^{N}\left(\xi_{t}^{N, 1}\right) w_{0}^{N}\left(\xi_{t}^{N, 2}\right) 1_{\left\{\tau^{N}>t\right\}}\right] d x_{1} d x_{2} .
\end{aligned}
$$

As before, either $\psi_{i} \geq 0$, or one side is integrable for the above to hold. A simple change of variables shows that (3.2) implies (for $\psi_{1}$ as above)

$$
E\left(X_{t}^{N}\left(\psi_{1}\right)\right)=E_{X_{0}^{N}}\left(\psi_{1}\left(\eta_{t}^{N}\right)\right):=\int_{\mathbb{R}^{d}} E_{\{x\}}^{N}\left(\psi_{1}\left(\eta_{t}^{N}\right)\right) X_{0}^{N}(d x) .
$$

Proposition 3.1.

(a) There exists $C_{3.5}>0$ such that for $s \geq 0$,

$$
\bar{m}_{s}^{N}(\mathbf{1})=m_{s}^{N}(\mathbf{1}) \leq C_{3.5} \begin{cases}X_{s}^{N}(\mathbf{1}) & \text { if } d \geq 3 \\ (\log N) X_{s}^{N}(\mathbf{1}) & \text { if } d=2\end{cases}
$$
$s \geq 0$,

(b) Assume $d=2$. If $0<\alpha<1$ then there exists $C_{3.6}=C_{3.6}(\alpha)>0$ such that for

$$
E\left(m_{s}^{N}(\mathbf{1})\right) \leq C_{3.6}\left(1+s^{-\alpha}\right) X_{0}^{N}(\mathbf{1}) .
$$

Proof. (a) The case $d \geq 3$ with $K=N^{d / 2-1}$ is straightforward. Using the definition of $\bar{m}_{s}^{N}$, Lemma 2.4(b) and the fact that $0 \leq w_{s}^{N} \leq 1$,

$$
\begin{array}{r}
m_{s}^{N}(\mathbf{1})=\bar{m}_{s}^{N}(\mathbf{1}) \leq \rho^{2} N^{d} K\left|B_{r}^{N}\right| \int_{\mathbb{R}^{d}} \int_{B_{r}^{N}(x)} w_{s}^{N}\left(z_{2}\right) d z_{2} d x=\rho^{2} N^{d}\left|B_{r}^{N}\right|^{2} X_{s}^{N}(\mathbf{1}) \\
=\rho^{2}\left|B_{r}\right|^{2} X_{s}^{N}(\mathbf{1}) .
\end{array}
$$

Now suppose $d=2$, with $K=\log N$. Write $\left(1-w_{s}^{N}\left(z_{1}\right)\right) w_{s}^{N}\left(z_{2}\right)=w_{s}^{N}\left(z_{2}\right)-w_{s}^{N}\left(z_{1}\right) w_{s}^{N}\left(z_{2}\right)$, and so conclude that

$$
m_{s}^{N}(\mathbf{1})=\bar{m}_{s}^{N}(\mathbf{1})=m_{s}^{N, 1}-m_{s}^{N, 2},
$$

where

$$
\begin{aligned}
& m_{s}^{N, 1}=\rho^{2} N^{2}(\log N)^{2} \int_{\mathbb{R}^{2}} \int_{B_{r}^{N}(x)} \int_{B_{r}^{N}(x)} w_{s}^{N}\left(z_{2}\right) d z_{1} d z_{2} d x \\
& m_{s}^{N, 2}=\rho^{2} N^{2}(\log N)^{2} \int_{\mathbb{R}^{2}} \int_{B_{r}^{N}(x)} \int_{B_{r}^{N}(x)} w_{s}^{N}\left(z_{1}\right) w_{s}^{N}\left(z_{2}\right) d z_{1} d z_{2} d x .
\end{aligned}
$$


Thus, $m_{s}^{N}(\mathbf{1}) \leq m_{s}^{N, 1}$, and

$$
\begin{aligned}
m_{s}^{N, 1} & =\rho^{2} N^{2}\left|B_{r}^{N}\right|(\log N) \int_{\mathbb{R}^{2}} \int_{B_{r}^{N}(x)} X_{s}^{N}\left(z_{2}\right) d z_{2} d x \\
& =\rho^{2} N^{2}\left|B_{r}^{N}\right|^{2}(\log N) X_{s}^{N}(\mathbf{1}) \\
& =\rho^{2}\left|B_{r}\right|^{2}(\log N) X_{s}^{N}(\mathbf{1}) .
\end{aligned}
$$

This completes the proof of (3.5).

(b) For $d=2$, using the two particle duality equation (3.3) with $\psi_{2} \equiv 1$, we have

$$
\begin{aligned}
E\left[m_{s}^{N, 2}\right]= & \rho^{2} N^{2}(\log N)^{2} \int_{\mathbb{R}^{2}} \int_{B_{r}^{N}(x)} \int_{B_{r}^{N}(x)} E\left[w_{s}^{N}\left(z_{1}\right) w_{s}^{N}\left(z_{2}\right)\right] d z_{1} d z_{2} d x \\
= & \rho^{2} N^{2}(\log N)^{2} \int_{\mathbb{R}^{2}} \int_{B_{r}^{N}(x)} \int_{B_{r}^{N}(x)}\left(E_{\left\{z_{1}, z_{2}\right\}}^{N}\left[w_{0}^{N}\left(\xi_{s}^{N, 1}\right) 1\left\{\tau^{N} \leq s\right\}\right]\right. \\
& \left.\quad+E_{\left\{z_{1}, z_{2}\right\}}^{N}\left[w_{0}^{N}\left(\xi_{s}^{N, 1}\right) w_{0}^{N}\left(\xi_{s}^{N, 2}\right) 1\left\{\tau^{N}>s\right\}\right]\right) d z_{1} d z_{2} d x \\
\geq & \rho^{2} N^{2}(\log N) \int_{\mathbb{R}^{2}} \int_{B_{r}^{N}(x)} \int_{B_{r}^{N}(x)} E_{\left\{z_{1}, z_{2}\right\}}^{N}\left[X_{0}^{N}\left(\xi_{s}^{N, 1}\right) 1\left\{\tau^{N} \leq s\right\}\right] d z_{1} d z_{2} d x \\
= & \rho^{2} N^{2}(\log N) \int_{\mathbb{R}^{2}} \int_{B_{r}^{N}} \int_{B_{r}^{N}} E_{\left\{x+z_{1}^{\prime}, x+z_{2}^{\prime}\right\}}^{N}\left[X_{0}^{N}\left(\xi_{s}^{N, 1}\right) 1\left\{\tau^{N} \leq s\right\}\right] d z_{1}^{\prime} d z_{2}^{\prime} d x \\
= & \rho^{2} N^{2}(\log N) \int_{\mathbb{R}^{2}} \int_{B_{r}^{N}} \int_{B_{r}^{N}} E_{\left\{z_{1}^{\prime}, z_{2}^{\prime}\right\}}^{N}\left[X_{0}^{N}\left(x+\xi_{s}^{N, 1}\right) 1\left\{\tau^{N} \leq s\right\}\right] d z_{1}^{\prime} d z_{2}^{\prime} d x,
\end{aligned}
$$

the last two equalities by a change of variables and translation invariance (1.7). Let $P_{\left\{U_{1}, U_{2}\right\}}(\cdot)=\int_{B_{r}} \int_{B_{r}} P_{\left\{z_{1}^{\prime}, z_{2}^{\prime}\right\}}(\cdot) d z_{1}^{\prime} d z_{2}^{\prime} /\left|B_{r}\right|^{2}$ so that $P_{\left\{U_{1}, U_{2}\right\}}(\tau>t)=\gamma_{e}(t)$. Integrating $x$ out (inside the expectation), we obtain

$$
\begin{aligned}
E\left[m_{s}^{N, 2}\right] & \geq \rho^{2} N^{2}(\log N) X_{0}^{N}(\mathbf{1}) \int_{B_{r}^{N}} \int_{B_{r}^{N}} P_{\left\{z_{1}^{\prime}, z_{2}^{\prime}\right\}}^{N}\left(\tau^{N} \leq s\right) d z_{1}^{\prime} d z_{2}^{\prime} \\
& =\rho^{2} N^{2}\left|B_{r}^{N}\right|^{2}(\log N) P_{\left\{U_{1}, U_{2}\right\}}(\tau \leq N s) X_{0}^{N}(\mathbf{1}) \\
& =\rho^{2}\left|B_{r}\right|^{2}(\log N)\left(1-\gamma_{e}(N s)\right) X_{0}^{N}(\mathbf{1}) .
\end{aligned}
$$

If we plug this bound and (3.8) into (3.7), we get

$$
E\left[m_{s}^{N}(\mathbf{1})\right] \leq \rho^{2}\left|B_{r}\right|^{2}(\log N) \gamma_{e}(N s) X_{0}^{N}(\mathbf{1})
$$

If $s \leq(\log N)^{-\alpha}$, then $(\log N) \gamma_{e}(N s) \leq \log N \leq s^{-1 / \alpha}$. By Proposition 1.1, if $s \geq$ $(\log N)^{-\alpha}$,

$$
(\log N) \gamma_{e}(N s) \leq(\log N) \gamma_{e}\left(\frac{N}{(\log N)^{\alpha}}\right) \rightarrow \gamma_{e}
$$

as $N \rightarrow \infty$. This implies that there is a $C>0$, depending on $\alpha$, such that for all $s \geq(\log N)^{-\alpha},(\log N) \gamma_{e}(N s) \leq C$. Plugging these bounds into (3.9) we obtain (3.6).

Corollary 3.2. For $d \geq 2$ and $T>0$ there exists $C_{3.10}(T)>0$ such that

$$
E\left[\sup _{t \leq T} X_{t}^{N}(\mathbf{1})^{2}\right] \leq 2 X_{0}^{N}(\mathbf{1})^{2}+C_{3.10}(T) X_{0}^{N}(\mathbf{1})
$$

Moreover $t \rightarrow X_{t}^{N}(\mathbf{1})$ is a non-negative square-integrable $\left(\mathcal{F}_{t}^{N}\right)$-martingale, and for any $\phi \in C_{0}^{3}\left(\mathbb{R}^{d}\right), t \rightarrow M_{t}^{N}(\phi)$ is also a square-integrable $\left(\mathcal{F}_{t}^{N}\right)$-martingale. 
Proof. By Proposition 2.2 and Lemma 2.3(a), $X_{t}^{N}(\mathbf{1})=X_{0}^{N}(\mathbf{1})+M_{t}^{N}(\mathbf{1})$, and so

$$
X_{t}^{N}(1)^{2} \leq 2 X_{0}^{N}(\mathbf{1})^{2}+2 M_{t}^{N}(\mathbf{1})^{2} .
$$

Now $X_{t}^{N}(\mathbf{1})$ is a non-negative local martingale which by (1.10) satisfies $E\left[X_{t}^{N}(\mathbf{1})\right]=$ $X_{0}^{N}(\mathbf{1})$, and so is a non-negative martingale. By Doob's $L^{2}$ submartingale inequality,

$$
E\left[\sup _{t \leq T} M_{t}^{N}(\mathbf{1})^{2}\right] \leq 4 E\left[\left\langle M^{N}(\mathbf{1})\right\rangle_{T}\right]=4 \int_{0}^{T} E\left[m_{s}^{N}(\mathbf{1})\right] d s .
$$

As we don't know the square integrability yet, the first inequality holds by considering a sequence of localizing stopping times and applying monotone convergence. By Proposition 3.1(a), for $d \geq 3$,

$$
\int_{0}^{T} E\left[m_{s}^{N}(\mathbf{1})\right] d s \leq C_{3.5} \int_{0}^{T} E\left[X_{s}^{N}(\mathbf{1})\right] d s=C_{3.5} T X_{0}^{N}(\mathbf{1}) .
$$

By Proposition 3.1(b), for $d=2$ and taking $\alpha=1 / 2$,

$$
\int_{0}^{T} E\left[m_{s}^{N}(\mathbf{1})\right] d s \leq C_{3.6}\left(\frac{1}{2}\right) X_{0}^{N}(\mathbf{1}) \int_{0}^{T}\left(1+s^{-1 / 2}\right) d s=C_{3.6}\left(\frac{1}{2}\right)\left(T+2 T^{1 / 2}\right) X_{0}^{N}(\mathbf{1}) .
$$

Combining the above bounds we obtain (3.10) and hence the next to last statement as well.

It is easy to repeat the above reasoning using Lemma 2.4(a) and see that

$$
E\left[\sup _{t \leq T} M_{t}^{N}(\phi)^{2}\right] \leq C_{\phi}[T+\sqrt{T}] X_{0}^{N}(\mathbf{1}) .
$$

This in turn shows that the local martingale $M^{N}(\phi)$ is in fact a square integrable martingale.

\section{Proof of main result}

The proof of Theorem 1.2 proceeds by taking limits as $N \rightarrow \infty$ in Proposition 2.2 to derive the martingale problem for the limiting super-Brownian motion. The main issue is the identification of the square function of the limiting martingale part and the key here is the following result:

Proposition 4.1. For all $A, T>0$, and $\phi \in C_{0}^{3}$,

$$
\sup _{X_{0}^{N}(\mathbf{1}) \leq A} E\left[\left.\sup _{0 \leq t \leq T}\left|\left\langle M^{N}(\phi)\right\rangle_{t}-\int_{0}^{t} \rho^{2}\right| B_{r}\right|^{2} \gamma_{e} X_{s}^{N}\left(\phi^{2}\right) d s \mid\right] \rightarrow 0 \text { as } N \rightarrow \infty .
$$

This will be proved in Section 7. In this section we will establish Theorem 1.2, assuming this result. If $S$ is a metric space, recall that a sequence of laws on $D\left(\mathbb{R}_{+}, S\right)$ is $C$-tight iff it is tight and all limit laws are continuous. $C$-tightness on $D\left(\mathbb{R}_{+}, S\right) \times C\left(\mathbb{R}_{+}, S\right)$ is then defined in the obvious manner. The first step is to prove:

Lemma 4.2. If $\phi \in C_{0}^{3}\left(\mathbb{R}^{d}\right)$, then $\left\{\left(X^{N}(\phi),\left\langle M^{N}(\phi)\right\rangle\right): N \geq 3\right\}$ is $C$-tight in $D\left(\mathbb{R}_{+}, \mathbb{R}\right) \times$ $C\left(\mathbb{R}_{+}, \mathbb{R}\right)$.

Proof. Let $\phi \in C_{0}^{3}\left(\mathbb{R}^{d}\right)$ and $A^{N}(t)=\int_{0}^{t} \rho^{2}\left|B_{r}\right|^{2} \gamma_{e} X_{s}^{N}\left(\phi^{2}\right) d s$. Then for $0 \leq s<t \leq T$,

$$
E\left(\left|A_{t}^{N}-A_{s}^{N}\right|^{2}\right) \leq C E\left(\sup _{u \leq T} X_{u}^{N}(\mathbf{1})^{2}\right)(t-s)^{2} \leq C(T)(t-s)^{2},
$$




\section{Convergence of SLFV to SBM}

by Corollary 3.2 and (1.18). Therefore, the collection of continuous increasing processes $\left\{A^{N}: N \geq 3\right\}$ is tight, and hence relatively compact, in $C\left(\mathbb{R}_{+}, \mathbb{R}\right)$ by Prohorov's theorem. It then follows from Proposition 4.1 that the sequence of continuous (recall (2.6)) increasing processes $\left\{\left\langle M^{N}(\phi)\right\rangle\right.$.:N $\left.\geq 3\right\}$ is relatively compact in $C\left(\mathbb{R}_{+}, \mathbb{R}\right)$, and so also tight by Prohorov's theorem again.

Next, recall $D^{N}(\phi)$ from Proposition 2.2. Lemma 2.3 implies that $D_{t}^{N}(\phi)=\int_{0}^{t} d_{s}^{N}(\phi) d s$, where

$$
\left|d_{s}^{N}(\phi)\right| \leq C_{\phi} X_{s}^{N}(\mathbf{1}) \quad \forall s \geq 0 .
$$

Therefore if $0 \leq s<t \leq T$, then by the above and Corollary 3.2,

$$
E\left(\left(D_{t}^{N}(\phi)-D_{s}^{N}(\phi)^{2}\right) \leq C_{\phi}^{2} \int_{s}^{t} \int_{s}^{t} E\left(X_{u}^{N}(\mathbf{1}) X_{v}^{N}(\mathbf{1})\right) d v d u \leq C(\phi, T)(t-s)^{2} .\right.
$$

This implies $\left\{D^{N}(\phi): N \geq 3\right\}$ is tight in $C\left(\mathbb{R}_{+}, \mathbb{R}\right)$. Lemma 2.1 and Proposition 2.2 imply

$$
\sup _{s \leq T}\left|\Delta M_{s}^{N}(\phi)\right|=\sup _{s \leq T}\left|\Delta X_{s}^{N}(\phi)\right| \rightarrow 0 \text { a.s. as } N \rightarrow \infty .
$$

Using (4.2) and the $C$-tightness of $\left\{\left\langle M^{N}(\phi)\right\rangle .: N \geq 3\right\}$, established above, in Theorem VI.4.13 and Proposition IV.3.26 of [20], we see that $\left\{M^{N}(\phi): N \geq 3\right\}$ is $C$-tight in $D\left(\mathbb{R}_{+}, \mathbb{R}\right)$. $C$-tightness of $\left\{X^{N}(\phi)\right\}$ now follows from the above, our assumption on the initial conditions $\left\{X_{0}^{N}\right\}$ (i.e., (1.18)), and the semimartingale decomposition in Proposition 2.2. Having obtained $C$-tightness of each component, the result is now immediate.

Proposition 4.3. For $d \geq 2$, under the assumptions of Theorem 1.2, the family $\left\{P\left(X^{N} \in \cdot\right): N \geq 3\right\}$ is $C$-tight in $D\left(\mathbb{R}_{+}, \mathcal{M}_{F}\left(\mathbb{R}^{d}\right)\right)$.

Proof. By the Kurtz-Jakubowski theorem (e.g. see Proposition 3.1 in [6]) it suffices to show:

1. For each $T, \varepsilon>0$ there is a compact set $K_{T, \varepsilon} \subset \mathbb{R}^{d}$ such that

$$
\limsup _{N \rightarrow \infty} P\left(\sup _{t \leq T} X_{t}^{N}\left(K_{T, \varepsilon}^{c}\right)>\varepsilon\right)<\varepsilon .
$$

2. For each $T>0$,

$$
\lim _{H \rightarrow \infty} \limsup _{N \rightarrow \infty} P\left(\sup _{t \leq T} X_{t}^{N}(\mathbf{1}) \geq H\right)=0 .
$$

3. For each $\phi \in C_{0}^{\infty}\left(\mathbb{R}^{d}\right)$,

$$
\left\{X_{.}^{N}(\phi), N \geq 3\right\} \text { is } C \text {-tight in } D\left(\mathbb{R}_{+}, \mathbb{R}\right) .
$$

The last (4.5) holds by Lemma 4.2, and (4.4) is immediate from Corollary 3.2. The compact containment (4.3) is proved exactly as for the voter model in Lemma 3.3 of [6]. The argument there will now use the semimartingale decomposition in Proposition 2.2, the convergence of the initial states from (1.18), Lemma 2.3, and first moment bounds which are immediate from (3.4). This completes the proof.

We are ready to turn to the main result.

Proof of Theorem 1.2. By Proposition 4.3 it suffices to show that every weak subsequential limit is the super-Brownian motion described in the Theorem. Fix $\phi \in C_{0}^{3}\left(\mathbb{R}^{d}\right)$. By 
Lemma 4.2 and Skorokhod's theorem, and then taking a further subsequence, we may assume that we are on a probability space where

$$
\left(X^{N_{k}},\left\langle M^{N_{k}}(\phi)\right\rangle\right) \rightarrow\left(X, A^{\phi}\right) \text { a.s. in } D\left(\mathbb{R}_{+}, \mathcal{M}_{F}\left(\mathbb{R}^{d}\right)\right) \times C\left(\mathbb{R}_{+}, \mathbb{R}\right) .
$$

Since the limit is continuous a.s. one has in fact a.s. uniform convergence on compact time intervals. It also follows from the above and Corollary 3.2 that

$$
\begin{array}{r}
\sup _{t \leq T}\left[\left|X_{t}^{N_{k}}(\mathbf{1})-X_{t}(\mathbf{1})\right|+\left|X_{t}^{N_{k}}(\phi)-X_{t}(\phi)\right|+\left|X_{t}^{N_{k}}\left(\phi^{2}\right)-X_{t}\left(\phi^{2}\right)\right|+\left|X_{t}^{N_{k}}(\Delta \phi)-X_{t}(\Delta \phi)\right|\right] \\
\rightarrow 0 \text { a.s. and in } L^{1} \text { as } k \rightarrow \infty \text { for all } T>0 .
\end{array}
$$

This and Proposition 4.1 show that

$$
A_{t}^{\phi}=\int_{0}^{t} \rho^{2}\left|B_{r}\right|^{2} \gamma_{e} X_{s}\left(\phi^{2}\right) d s \text { for all } t \geq 0 .
$$

It follows from (4.6), (4.7), and Proposition 4.1 that

$$
\sup _{t \leq T}\left|\left\langle M^{N_{k}}(\phi)\right\rangle_{t}-A_{t}^{\phi}\right| \rightarrow 0 \text { a.s. and in } L^{1} \text { as } k \rightarrow \infty \text { for all } T>0,
$$

Lemma 2.3, Corollary 3.2, and (4.7) imply

$$
\sup _{t \leq T}\left|D_{t}^{N_{k}}(\phi)-\int_{0}^{t} \rho\right| B_{r}\left|\bar{\sigma}^{2} X_{s}(\Delta \phi / 2) d s\right| \rightarrow 0 \text { as } k \rightarrow \infty \text { a.s. and in } L^{1} \text { as } k \rightarrow \infty \text {. }
$$

Define an a.s. continuous process by $M_{t}(\phi)=X_{t}(\phi)-X_{0}(\phi)-\int_{0}^{t} \rho\left|B_{r}\right| \bar{\sigma}^{2} X_{s}(\Delta \phi / 2) d s$. Then the above, the convergence of the initial conditions in (1.18), and the semimartingale decomposition for $X^{N}(\phi)$ in Proposition 2.2 show that

$$
\sup _{t \leq T}\left|M_{t}^{N_{k}}(\phi)-M_{t}(\phi)\right| \rightarrow 0 \text { in } L^{1} \text { and a.s. as } k \rightarrow \infty \text { for all } T>0 .
$$

Since $M^{N_{k}}(\phi)$ is an $\left(\mathcal{F}_{t}^{X^{N_{k}}}\right)$-martingale by Corollary 3.2, it follows from the above that $M(\phi)$ is a continuous martingale and a standard argument (e.g. see the proof of Theorem 3.5 in [6]) shows it is in fact an $\left(\mathcal{F}_{t}^{X}\right)$-martingale.

Recalling (1.12), (4.8), and the value of $b$ in Theorem 1.2 , it remains to identify the square function of $M(\phi)$ as $A^{\phi}$ by showing

$$
M_{t}(\phi)^{2}-A_{t}(\phi) \text { is a local martingale. }
$$

For $d \geq 3$ this is fairly easy, but we give a stopping argument to include the more delicate 2-dimensional case. For $J \in \mathbb{N}$ define

$$
T_{J}^{N}=\inf \left\{t:\left|M_{t}^{N}(\phi)\right| \geq J\right\}, \quad T_{J}=\inf \left\{t:\left|M_{t}(\phi)\right| \geq J\right\} .
$$

The convergence in (4.11) readily shows that

$$
\liminf _{k \rightarrow \infty} T_{J}^{N_{k}} \geq T_{J} \quad \forall J \in \mathbb{N} \text { a.s. }
$$

We claim that

$$
\lim _{k \rightarrow \infty} \sup _{t \leq T}\left[\left|M_{t \wedge T_{J}^{N_{k}}}^{N_{k}}(\phi)-M_{t \wedge T_{J}}(\phi)\right|+\left|\left\langle M^{N_{k}}(\phi)\right\rangle_{t \wedge T_{J}^{N_{k}}}-A_{t \wedge T_{J}}^{\phi}\right|\right]=0 \text { for all } T>0 \text { a.s. }
$$


The reason there is an issue here is that we do not know whether or not $\lim _{k} T_{J}^{N_{k}}=T_{J}$ a.s. It follows from (4.13) that for $t \leq T_{J}$ we have $\lim _{k} T_{J}^{N_{k}} \wedge t=t=T_{J} \wedge t$ (the convergence is uniform for $t \leq T_{J} \wedge T$ for any fixed $T$ ) and therefore by (4.11) and (4.9),

$$
\lim _{k \rightarrow \infty} \sup _{t \leq T_{J} \wedge T}\left[\left|M_{t \wedge T_{J}^{N_{k}}}^{N_{k}}(\phi)-M_{t \wedge T_{J}}(\phi)\right|+\left|\left\langle M^{N_{k}}(\phi)\right\rangle_{t \wedge T_{J}^{N_{k}}}-A_{t \wedge T_{J}}^{\phi}\right|\right]=0 \text { for all } T>0 \text { a.s. }
$$

A simple calculation using (4.13) shows that $(\sup \emptyset:=0)$ with probability one for any $T>0$,

$$
\begin{aligned}
\limsup _{k \rightarrow \infty} & \sup _{T_{J}^{N_{k}} \leq t \leq T}\left[\left|M_{t \wedge T_{J}^{N_{k}}}^{N_{k}}(\phi)-M_{t \wedge T_{J}}(\phi)\right|+\left|\left\langle M^{N_{k}}(\phi)\right\rangle_{t \wedge T_{J}^{N_{k}}}-A_{t \wedge T_{J}}^{\phi}\right|\right] \\
& =\limsup _{k \rightarrow \infty} 1\left(T_{J}^{N_{k}} \leq T\right)\left[\left|M_{T_{J}^{N_{k}}}^{N_{k}}(\phi)-M_{T_{J}}(\phi)\right|+\left|\left\langle M^{N_{k}}(\phi)\right\rangle_{T_{J}^{N_{k}}}-A_{T_{J}}^{\phi}\right|\right] .
\end{aligned}
$$

In view of the above and (4.15), to prove (4.14) it suffices to show that for $T>0$ fixed,

$$
\limsup _{k \rightarrow \infty} \sup _{T_{J}<t \leq T_{J}^{N_{k}} \wedge T}\left[\left|M_{t}^{N_{k}}(\phi)-M_{T_{J}}(\phi)\right|+\left|\left\langle M^{N_{k}}(\phi)\right\rangle_{t}-A_{T_{J}}^{\phi}\right|\right]=0 \text { a.s. }
$$

By (4.9) and (4.11) this would follow from

$$
\limsup _{k \rightarrow \infty} \sup _{T_{J}<t \leq T_{J}^{N_{k}} \wedge T}\left[\left|M_{t}(\phi)-M_{T_{J}}(\phi)\right|+\left|A_{t}^{\phi}-A_{T_{J}}^{\phi}\right|\right]=0 \text { a.s. }
$$

For this we will use the following lemma, whose proof is deferred to the end of this section.

Lemma 4.4. With probability one, for all $0 \leq s<t, M_{u}(\phi)=M_{s}(\phi)$ for all $u \in[s, t]$ implies that $A_{t}^{\phi}=A_{s}^{\phi}$.

By the Dubins-Schwarz theorem we may assume $M_{t}(\phi)=B\left(\langle M(\phi)\rangle_{t}\right)$ for some Brownian motion, $B$, on our probability space. We let $T_{J}^{B}$ and $T_{J+}^{B}$ denote the exit times of $B$ from $(-J, J)$ and $[-J, J]$, respectively. On $\left\{\lim \sup _{k} T_{J}^{N_{k}} \wedge T \leq T_{J}\right\}$, (4.18) follows from the a.s. continuity of $M(\phi)$ and $A^{\phi}$. So assume $\omega$ is in $\left\{\lim \sup _{k} T_{J}^{N_{k}} \wedge T>T_{J}\right\}$ and also outside of a null set so that:

(i) (4.9) and (4.11) hold;

(ii) for all $s<t,\langle M(\phi)\rangle_{t}=\langle M(\phi)\rangle_{s}$ implies $M_{u}(\phi)=M_{s}(\phi)$ for all $u \in[s, t]$;

(iii) the conclusion of Lemmas 2.1 and 4.4 hold;

(iv) $T_{J}^{B}=T_{J+}^{B}$.

Use the conclusion of Lemma 2.1 and our choice of $\omega$ to see that $\forall t \in\left[0, \lim _{\sup _{k}} T_{J}^{N_{k}} \wedge T\right)$,

$$
\left|B\left(\langle M(\phi)\rangle_{t}\right)\right|=\left|M_{t}(\phi)\right|=\lim _{k}\left|M_{t}^{N_{k}}(\phi)\right| \leq \underset{k}{\limsup } J+C \frac{\log N_{k}}{N_{k}}=J .
$$

We easily see that $T_{J}^{B}=\left\langle(M(\phi)\rangle_{T_{J}}\right.$, and so the above shows that $|B(u)| \leq J$ on $\left[T_{J}^{B},\langle M(\phi)\rangle\left(\limsup _{k} T_{J}^{N_{k}} \wedge T\right)\right.$ ), so the fact that $T_{J+}^{B}=T_{J}^{B}$ (by our choice of $\omega$ ) implies this interval must be empty. We conclude that $\langle M(\phi)\rangle\left(\limsup _{k} T_{J}^{N_{k}} \wedge T\right)=\left\langle(M(\phi)\rangle\left(T_{J}\right)\right.$, which by our choice of $\omega$ implies that $M_{u}(\phi)=M_{T_{J}}(\phi)$ for all $u \in\left[T_{J}, \lim _{\sup _{k}} T_{J}^{N_{k}} \wedge T\right]$. Lemma 4.4 (and again our choice of $\omega$ ) shows that this gives $A_{u}^{\phi}=A_{T_{J}}^{\phi}$ for all $u \in$ $\left[T_{J}, \lim \sup _{k} T_{J}^{N_{k}} \wedge T\right]$. We have proved (4.18), and hence completed the derivation of (4.14).

Turning at last to (4.12), we see from Lemma 2.1 and Proposition 2.2 that

$$
\sup _{t}\left|M^{N_{k}}(\phi)_{T_{J}^{N_{k}} \wedge t}\right| \leq J+C_{\phi} \frac{\log N_{k}}{N_{k}} \leq C(J, \phi) \text { for all } k .
$$


This, together with the $L^{1}$ convergence of $\left\langle M^{N_{k}}(\phi)\right\rangle_{t}$ to $A_{t}^{\phi}$ from (4.9), implies that $\left\{\sup _{t \leq T}\left|\left[M^{N_{k}}(\phi)_{T_{J}^{N_{k} \wedge t}}\right]^{2}-\left\langle M^{N_{k}}(\phi)\right\rangle_{T_{J}^{N_{k} \wedge t}}\right|: k \in \mathbb{N}\right\}$ is uniformly integrable. From (4.14) and the above we can conclude that for each $t \geq 0$,

$$
\begin{aligned}
N_{T_{J}^{N_{k}} \wedge t}^{k} & :=\left(M^{N_{k}}(\phi)_{T_{J}^{N_{k}} \wedge t}\right)^{2}-\left\langle M^{N_{k}}(\phi)\right\rangle_{T_{J}^{N_{k}} \wedge t} \\
& \rightarrow M(\phi)_{T_{J} \wedge t}^{2}-A_{T_{J} \wedge t}^{\phi} \text { in } L^{1} \text { and a.s. }
\end{aligned}
$$

As $N_{T_{J}^{N_{k}} \wedge t}^{k}$ is a martingale (Corollary 3.2), this implies that $N_{t}=M(\phi)_{T_{J} \wedge t}^{2}-A_{T_{J} \wedge t}^{\phi}$ is a martingale for all $J$ which establishes (4.12) and so completes the proof.

Proof of Lemma 4.4. By continuity it suffices to prove the result for a fixed pair of times $0 \leq s<t$. For $J, k, n \in \mathbb{N}$, define

$$
U_{n}^{J, k}=\inf \left\{u \geq s:\left|\left(M^{N_{k}}(\phi)\left(T_{J}^{N_{k}} \wedge u\right)\right)^{2}-\left(M^{N_{k}}(\phi)\left(T_{J}^{N_{k}} \wedge s\right)\right)^{2}\right| \geq n^{-1}\right\} \quad(\inf \emptyset=\infty) .
$$

It follows from our jump bounds in (2.7) that

$$
\left|\Delta\left(M_{s}^{N_{k}}(\phi)^{2}\right)\right| \leq 2 J\|\phi\|_{\infty} C \frac{\log N_{k}}{N_{k}} \quad \text { for all } s \geq 0 .
$$

Recalling that $M^{N}(\phi)$ is a square integrable martingale (from Corollary 3.2), we have by optional stopping,

$$
\begin{aligned}
& E\left(\left\langle M^{N_{k}}(\phi)\right\rangle_{U_{n}^{k, J} \wedge T_{J}^{N_{k}} \wedge t}-\left\langle M^{N_{k}}(\phi)\right\rangle_{T_{J}^{N_{k}} \wedge s}\right) \\
& \quad=E\left(M^{N_{k}}(\phi)\left(U_{n}^{k, j} \wedge T_{J}^{N_{k}} \wedge t\right)^{2}-M^{N_{k}}(\phi)\left(U_{n}^{k, j} \wedge T_{J}^{N_{k}} \wedge s\right)^{2}\right) \\
& \quad \leq \frac{1}{n}+2 J\|\phi\|_{\infty} C \frac{\log N_{k}}{N_{k}} .
\end{aligned}
$$

Next use (4.13), and the convergence in (4.9) and (4.11), together with Fatou's lemma, to see that

$$
\begin{aligned}
E( & \left.\left(A_{t}^{\phi}-A_{s}^{\phi}\right) 1\left(T_{J}>t\right) 1\left(\sup _{s \leq u \leq t}\left|M_{u}(\phi)^{2}-M_{s}(\phi)^{2}\right|<\frac{1}{2 n}\right)\right) \\
\leq & E\left(\liminf _{k \rightarrow \infty}\left(\left\langle M^{N_{k}}(\phi)\right\rangle_{t}-\left\langle M^{N_{k}}(\phi)\right\rangle_{s}\right) 1\left(T_{J}^{N_{k}}>t\right) 1\left(\sup _{s \leq u \leq t}\left|M_{u}^{N_{k}}(\phi)^{2}-M_{s}^{N_{k}}(\phi)^{2}\right|<\frac{1}{n}\right)\right) \\
\leq & \liminf _{k \rightarrow \infty} E\left(\left(\left\langle M^{N_{k}}(\phi)\right\rangle_{t \wedge T_{J}^{N_{k}} \wedge U_{n}^{k, J}}-\left\langle M^{N_{k}}(\phi)\right\rangle_{s \wedge T_{J}^{N_{k}}}\right) 1\left(T_{J}^{N_{k}}>t\right)\right. \\
\quad & \left.\times 1\left(\sup _{s \leq u \leq t}\left|M_{u}^{N_{k}}(\phi)^{2}-M_{s}^{N_{k}}(\phi)^{2}\right|<\frac{1}{n}\right)\right) \\
\leq & \frac{1}{n},
\end{aligned}
$$

where the last is by (4.20). Let $J \rightarrow \infty$ and then $n \rightarrow \infty$ to prove the result for $s<t$ fixed, as required.

\section{Random walk preliminaries}

Recall that $\left(\xi_{t}^{1}, \xi_{t}^{2}\right)$ is the two particle dual with initial state $\left(x_{1}, x_{2}\right)$. We will need to work with the difference process,

$$
\tilde{\xi}_{t}=\xi_{t}^{1}-\xi_{t}^{2},
$$

which is a Markov process starting at $x_{1}-x_{2}$. When $\left|\tilde{\xi}_{t}\right|>2 r$, it makes the same transitions as a rate $2 \rho\left|B_{r}\right|$ random walk with jump distribution that of $\bar{U}$, with density 
$h_{\bar{U}}(z)$ given in (1.3). We will need basic information about this random walk, as well as a way to compare $\tilde{\xi}_{t}$ to it.

Throughout the paper, $Y_{t}=Y_{t}^{x}$ will denote a rate $2 \rho\left|B_{r}\right|$ random walk with jump distribution that of $\bar{U}$ starting at $x$ under $P_{x}$. That is, $Y_{t}^{x}$ will be the pure-jump Markov process on $\mathbb{R}^{d}$ with generator

$$
\mathcal{A}^{Y} f(x)=2 \rho\left|B_{r}\right| \int_{B_{2 r}(0)}(f(x+z)-f(x)) h_{\bar{U}}(z) d z
$$

defined for suitable $f$. We will often make use of the Poisson process construction

$$
Y_{t}^{0}=S_{N_{t}}, \quad t \geq 0,
$$

where $N_{t}$ is a rate $\lambda=2 \rho\left|B_{r}\right|$ Poisson process on $[0, \infty)$ which is independent of the iid random variables $\bar{U}_{1}, \bar{U}_{2}, \ldots$ which have the same law as $\bar{U}$, and $S_{n}=\bar{U}_{1}+\cdots \bar{U}_{n}, n \geq 1$ $\left(S_{0}=0\right)$. We will often write $Y_{t}$ for $Y_{t}^{0}$ and $Y_{t}^{x}$ for $x+Y_{t}^{0}$, where $x$ may be random. Recall from (1.4) that $=E\left[|\bar{U}|^{2}\right]=d \bar{\sigma}^{2}$.

Lemma 5.1. (a) There is a constant $C_{5.2}>0$ such that for all $t \geq 0$ and Borel $B \subset \mathbb{R}^{d}$,

$$
\begin{aligned}
& P\left(Y_{t}^{\bar{U}} \in B\right) \leq \frac{C_{5.2}|B|}{1+t^{d / 2}}, \text { and } \\
& P_{x}\left(Y_{t} \in B\right) \leq e^{-2 \rho\left|B_{r}\right| t} 1_{B}(x)+\frac{C_{5.2}|B|}{1+t^{d / 2}} \text { for all } x \in \mathbb{R}^{d} .
\end{aligned}
$$

In particular, for all $x \in \mathbb{R}^{d}, t>0$, and nonnegative Borel $f$,

$$
E_{x}\left[f\left(\frac{Y_{t}}{\sqrt{t}}\right)\right] \leq e^{-2 \rho\left|B_{r}\right| t}\|f\|_{\infty}+C_{5.2} \int_{\mathbb{R}^{d}} f(z) d z
$$

(b) For all $t \geq 0, E_{0}\left[\left|Y_{t}\right|^{2}\right]=2 \rho\left|B_{r}\right| d \bar{\sigma}^{2} t$. For $k \in \mathbb{N}$ there is a constant $C_{5.4}=C_{5.4}(k)>0$ such that

$$
E_{0}\left[\sup _{s \leq t}\left|Y_{s}\right|^{2 k}\right] \leq C_{5.4} t^{k} \text { for all } t \geq 1 \text {. }
$$

(c) For $k \in \mathbb{N}$ there is a constant $C_{5.5}=C_{5.5}(k)>0$

$$
P_{0}\left(\sup _{s \leq t}\left|Y_{s}\right| \geq a\right) \leq C_{5.5} \frac{t^{k}}{a^{2 k}} \text { for all } a>0, t \geq 1 .
$$

Proof. (a) According to Theorem 19.1 of [3], there is a uniform bound on the densities of $S_{n} / \sqrt{n}, n=1,2, \ldots$, so that

$$
P\left(\frac{S_{n}}{\sqrt{n}} \in B\right) \leq C|B| \quad \forall n \geq 1, \text { Borel } B \subset \mathbb{R}^{d} .
$$

By a standard large deviations estimate, for $0<\alpha<1$,

$$
P\left(N_{t} \leq \alpha \lambda t\right) \leq \exp \left(-c_{\alpha} \lambda t\right)
$$

where $c_{\alpha}=1-\alpha+\alpha \log \alpha>0$. Using $Y_{t}^{x}=x+S_{N_{t}}$ and the density bound (5.6),

$$
\begin{aligned}
P\left(Y_{t}^{x} \in B\right) & =e^{-\lambda t} 1_{B}(x)+\sum_{n=1}^{\infty} e^{-\lambda t} \frac{(\lambda t)^{n}}{n !} P\left(\frac{S_{n}}{\sqrt{n}} \in \frac{B-x}{\sqrt{n}}\right) \\
& \leq e^{-\lambda t} 1_{B}(x)+C\left\{\sum_{1 \leq n<\lambda t / 2}+\sum_{n \geq 1 \vee(\lambda t / 2)}\right\} e^{-\lambda t} \frac{(\lambda t)^{n}}{n !} \frac{|B|}{n^{d / 2}} \\
& \leq e^{-\lambda t} 1_{B}(x)+C|B|\left(P\left(N_{t}<\lambda t / 2\right)+E\left[1\left\{N_{t} \geq 1 \vee(\lambda t / 2)\right\} N_{t}^{-d / 2}\right]\right) \\
& \leq e^{-\lambda t} 1_{B}(x)+C|B|\left(e^{-c_{1 / 2} \lambda t}+(1 \vee(\lambda t / 2))^{-d / 2}\right) \\
& \leq e^{-\lambda t} 1_{B}(x)+C|B|(1+t)^{-d / 2},
\end{aligned}
$$


where we have used the large deviation bound with $\alpha=1 / 2$. This proves (a) for $Y$ starting at $x$. The result for $Y_{t}^{\bar{U}}$ follows from the observation that $Y_{t}^{\bar{U}}$ has the same law as $S_{N(t)+1}$ and a slight alteration in the above calculation.

(b) It is easy to see from the representation $Y_{t}^{0}=S_{N_{t}}$ that $E_{0}\left[\left|Y_{t}\right|^{2}\right]=2 \rho\left|B_{r}\right| t E\left[|\bar{U}|^{2}\right]$. Now consider $k \in \mathbb{N}$. There is a constant $C_{k}>0$ such that $E\left(\max _{m \leq n}\left|S_{m}\right|^{2 k}\right) \leq C_{k} n^{k}$. To see this we switch to component notation, and write $\bar{U}_{j}=\left(\bar{U}_{j}^{(1)}, \ldots, \bar{U}_{j}^{(d)}\right)$ and $S_{n}=$ $\left(S_{n}^{(1)}, \ldots, S_{n}^{(d)}\right)$, where $S_{n}^{(j)}=\sum_{i=1}^{n} \bar{U}_{i}^{(j)}$. Then

$$
E\left[\max _{m \leq n}\left|S_{m}\right|^{2 k}\right]=E\left[\max _{m \leq n}\left(\sum_{j=1}^{d}\left|S_{m}^{(j)}\right|^{2}\right)^{k}\right] \leq d^{k-1} E\left[\sum_{j=1}^{d} \max _{m \leq n}\left|S_{n}^{(j)}\right|^{2 k}\right]=d^{k} E\left[\max _{m \leq n}\left|S_{m}^{(1)}\right|^{2 k}\right] .
$$

Now $S_{n}^{(1)}$ is a sum of bounded, mean zero independent random variables, so a martingale square function argument (e.g. see Theorem 21.1 of [4]) shows that for each $k \geq 1$ there is a constant $C_{k}=C_{k}(r)>0$ such that $E\left[\max _{m \leq n}\left|S_{m}^{(1)}\right|^{2 k}\right] \leq C_{k} n^{k}$ for all $n \in \mathbb{N}$. This implies

$$
E\left[\left|Y_{t}\right|^{2 k}\right]=\sum_{n=1}^{\infty} e^{-\lambda t} \frac{(\lambda t)^{n}}{n !} E\left[\left|S_{n}\right|^{2 k}\right] \leq C d^{k+1}\left(\sum_{n=1}^{k-1} e^{-\lambda t} \frac{(\lambda t)^{n}}{n !} n^{k}+\sum_{n=k}^{\infty} e^{-\lambda t} \frac{(\lambda t)^{n}}{n !} n^{k}\right) .
$$

The first sum is bounded by $(k-1)^{k}$. The second sum is bounded by

$$
(\lambda t)^{k} \sum_{n=k}^{\infty} e^{-\lambda t} \frac{(\lambda t)^{n-k}}{(n-k) !} \prod_{j=1}^{k} \frac{n}{n-j+1} \leq(\lambda t)^{k} k^{k} .
$$

This proves (5.4) for $t \geq 1$.

(c) This is immediate from (b) and Markov's inequality.

For $a, A>0$, define the hitting times

$$
t_{a}=\inf \left\{s \geq 0:\left|Y_{s}\right| \leq a\right\} \text { and } T_{A}=\inf \left\{s \geq 0:\left|Y_{s}\right| \geq A\right\} .
$$

Proposition 5.2. Assume $d \geq 3$ and $2 r<a<|x|$. Then

$$
P_{x}\left(t_{a}<\infty\right) \leq\left(\frac{a}{|x|}\right)^{d-2}
$$

Proof. Let $A>|x|$. By radial symmetry and (5.1), $f(x)=|x|^{2-d}$ is a harmonic function for $Y$. If we let $\sigma=t_{a} \wedge T_{A}$, then $\left|Y_{s \wedge \sigma}\right|^{2-d}$ is a bounded martingale (recall $a>2 r$ ), and so

$$
|x|^{2-d}=E_{x}\left[\left|Y_{t_{a}}\right|^{2-d} 1\left(t_{a}<T_{A}\right)\right]+E_{x}\left[\left|Y_{T_{A}}\right|^{2-d} 1\left(T_{A}<t_{a}\right)\right] .
$$

Clearly $\left|Y_{T_{A}}\right|^{2-d} \leq A^{2-d}$ if $T_{A}<t_{a}$, and $\left|Y_{t_{a}}\right|^{2-d} \geq a^{2-d}$ if $t_{a}<T_{A}$. This means that if we let $A \rightarrow \infty$ in (5.9), then

$$
|x|^{2-d} \geq E_{x}\left[a^{2-d} 1\left(t_{a}<\infty\right)\right]=a^{2-d} P_{x}\left(t_{a}<\infty\right),
$$

proving (5.8).

Lemma 5.3. Assume $d=2$. If $A>2$ and $2 r<a<|x|<A$, then

$$
\begin{gathered}
P_{x}\left(T_{A}<t_{a}\right) \leq \frac{\log |x|-\log (a-2 r)}{\log A-\log (a-2 r)}, \\
P_{x}\left(t_{a}<T_{A}\right) \leq \frac{\log (A+2 r)-\log |x|}{\log (A+2 r)-\log a},
\end{gathered}
$$

and

$$
\lim _{A \rightarrow \infty}(\log A) P_{x}\left(T_{A}<t_{a}\right)=\log |x|-E_{x}\left[\log \left|Y_{t_{a}}\right|\right]
$$




\section{Convergence of SLFV to SBM}

Proof. By radial symmetry and (5.1), $\log |x|$ is a harmonic function for $Y$. If $\sigma=t_{a} \wedge T_{A}$ as before then $\log \left|Y_{s \wedge \sigma}\right|$ is a bounded martingale, and

$$
\log |x|=E_{x}\left[\log \left|Y_{t_{a}}\right| ; t_{a}<T_{A}\right]+E_{x}\left[\log \left|Y_{T_{A}}\right| ; T_{A}<t_{a}\right] .
$$

Using $\left|Y_{T_{A}}\right| \geq A$ and $\left|Y_{t_{a}}\right|>a-2 r$ in the above gives

$$
\log |x| \geq(\log (a-2 r))\left(1-P_{x}\left(T_{A}<t_{a}\right)\right)+(\log A) P_{x}\left(T_{A}<t_{a}\right) .
$$

Rearranging gives (5.10). A similar argument yields (5.11).

For (5.12), rearranging (5.13) gives

$$
\begin{aligned}
|\log | x \mid & \left.-E_{x}\left[\log \left|Y_{t_{a}}\right| ; t_{a}<T_{A}\right)\right]-(\log A) P_{x}\left(T_{A}<t_{a}\right) \mid \\
& =E_{x}\left[\left(\log \left|Y_{T_{A}}\right|-\log A\right) 1\left(T_{A}<t_{a}\right)\right] \leq \log \left(\frac{A+2 r}{A}\right) \rightarrow 0
\end{aligned}
$$

as $A \rightarrow \infty$. Also, by recurrence and bounded convergence, $E_{x}\left[\log \left|Y_{t_{a}}\right| ; t_{a}<T_{A}\right] \rightarrow$ $E_{x}\left[\log \left|Y_{t_{a}}\right|\right]$ as $A \rightarrow \infty$, which means that (5.12) must hold.

Lemma 5.4. Assume $d=2$. There is a constant $C_{5.14}>0$ such that for $A>2$ and $|x|<A / 2$,

$$
P_{x}\left(T_{A} \notin\left[\frac{A^{2}}{\log A}, A^{2} \log A\right]\right) \leq C_{5.14} /(\log A)^{2} .
$$

Proof. By (5.5) with $k=2$, for all $x, A$ as in the Lemma,

$$
\begin{aligned}
P_{x}\left(T_{A} \leq \frac{A^{2}}{\log A}\right) & =P_{0}\left(\sup _{s \leq A^{2} / \log A}\left|x+Y_{s}\right| \geq A\right) \leq P_{0}\left(\sup _{s \leq A^{2} / \log A}\left|Y_{s}\right|>A / 2\right) \\
& \leq C_{5.5} \frac{\left(A^{2} / \log A\right)^{2}}{(A / 2)^{4}}=16 C_{5.5} /(\log A)^{2} .
\end{aligned}
$$

To handle $P_{x}\left(T_{A} \geq A^{2} \log A\right)$ we must first estimate $E_{x}\left(T_{A}^{2}\right)$. Let $\sigma_{2}=E\left(|\bar{U}|^{2}\right)$, $\sigma_{4}=E\left(|\bar{U}|^{4}\right)$ and $\lambda=2 \rho\left|B_{r}\right|$, and define the functions

$$
\begin{aligned}
& u_{2}(t, y)=|y|^{2}-\lambda \sigma_{2} t, \\
& u_{4}(t, y)=|y|^{4}-4 \lambda \sigma_{2}|y|^{2} t+2\left(\lambda \sigma_{2}\right)^{2} t^{2}-\lambda \sigma_{4} t .
\end{aligned}
$$

It is a straightforward calculation to check that both $u=u_{2}$ and $u=u_{4}$ satisfy

$$
\frac{\partial u}{\partial t}+\mathcal{A}^{Y} u \equiv 0 .
$$

This and the fact that for $p=2,4,|y|^{p}$, and $\mathcal{A}^{Y}\left(|y|^{p}\right)$ are bounded on $\{|y| \leq A+2 r\}$, so that $u_{p}\left(t \wedge T_{A}, Y_{t \wedge T_{A}}\right)$ is uniformly bounded for $t \leq t_{0}$, easily imply that both $u_{2}\left(t \wedge T_{A}, Y_{t \wedge T_{A}}\right)$ and $u_{4}\left(t \wedge T_{A}, Y_{t \wedge T_{A}}\right)$ are martingales. Therefore $E_{0}\left[u_{2}\left(t \wedge T_{A}, Y_{t \wedge T_{A}}\right)\right]=0$, and since $\left|Y_{T_{A}}\right| \leq A+2 r$

$$
\lambda \sigma_{2} E_{0}\left[T_{A}\right]=\lim _{t \rightarrow \infty} \lambda \sigma_{2} E_{0}\left[T_{A} \wedge t\right]=\lim _{t \rightarrow \infty} E_{0}\left[\left(Y_{T_{A} \wedge t}\right)^{2}\right] \leq(A+2 r)^{2} .
$$

Now, since $E_{0}\left[u_{4}\left(T_{A} \wedge t, Y_{T_{A} \wedge t}\right)\right]=0$,

$$
\begin{aligned}
2\left(\lambda \sigma_{2}\right)^{2} E_{0}\left[\left(T_{A} \wedge t\right)^{2}\right] & =-E_{0}\left[\left|Y_{T_{A} \wedge t}\right|^{4}\right]+4 \lambda \sigma_{2} E_{0}\left[\left(T_{A} \wedge t\right)\left|Y_{T_{A} \wedge t}\right|^{2}\right]+\lambda \sigma_{4} E_{0}\left[T_{A} \wedge t\right] \\
& \leq 4 \lambda \sigma_{2}(A+2 r)^{2} E_{0}\left[T_{A}\right]+\lambda \sigma_{4} E_{0}\left[T_{A}\right] \\
& \leq 4(A+2 r)^{4}+\frac{\sigma_{4}}{\sigma_{2}}(A+2 r)^{2},
\end{aligned}
$$




\section{Convergence of SLFV to SBM}

where we have used (5.16). Let $t \rightarrow \infty$ on the left-hand side of the above to conclude that

$$
E_{0}\left[T_{A}^{2}\right] \leq C(A+2 r)^{4} \text { for all } A>1 .
$$

On account of this bound and Markov's inequality, we have for $|x|<A / 2$ and $A>2$,

$$
P_{x}\left(T_{A}>A^{2} \log A\right) \leq P_{0}\left(T_{3 A / 2}>A^{2} \log A\right) \leq E_{0}\left[T_{3 A / 2}^{2}\right] /\left(A^{2} \log A\right)^{2} \leq C /(\log A)^{2} .
$$

Together with (5.15) this proves (5.14).

The following technical result will play a key role in the proof of Lemma 6.5 below.

Lemma 5.5. Assume $d=2$. For $\alpha, \beta>0$ and $t>0$ there is a constant $C_{5.18}=$ $C_{5.18}(\alpha, \beta, t) \geq 1$ such that if $(\log N)^{-\beta} \leq s \leq t$ and $w \in \mathbb{R}^{2}$ satisfies $0<|w| \leq(\log N)^{-\alpha}$, then

$$
P_{w \sqrt{N}}\left(t_{3 r}<N s\right) \leq C_{5.18} \frac{\log (1 /|w|)}{\log N} .
$$

Proof. We may suppose $|w|>3 r / \sqrt{N}$, because otherwise $C_{5.18}$ can be chosen large enough so that the right side of (5.18) is at least one. Now for any $A>|w| \sqrt{N}$,

$$
P_{w \sqrt{N}}\left(t_{3 r}<N s\right) \leq P_{w \sqrt{N}}\left(t_{3 r}<T_{A}\right)+P_{w \sqrt{N}}\left(T_{A} \leq N s\right) .
$$

To handle the first term, we apply Lemma 5.3 with $x=w \sqrt{N}$ and $a=3 r$,

$$
P_{w \sqrt{N}}\left(t_{3 r}<T_{A}\right) \leq \frac{\log \left(\frac{A+2 r}{w \sqrt{N}}\right)}{\log \left(\frac{A+2 r}{3 r}\right)} .
$$

Now set $A=|w| \sqrt{N}+\sqrt{N s}(\log N)^{\alpha}$. Using $s \leq t$ and $|w| \leq(\log N)^{-\alpha}$, and taking $N \geq N_{0}(t)$, we see that for some $C(t)>0$,

$$
\log \left(\frac{A+2 r}{|w| \sqrt{N}}\right) \leq \log \left(1+\frac{2 \sqrt{t}(\log N)^{\alpha}}{|w|}\right) \leq \log \left(1+\frac{2 \sqrt{t}}{|w|^{2}}\right) \leq C(t) \log (1 /|w|) .
$$

Using $s \geq(\log N)^{-\beta}$ gives for $N \geq N_{1}(\alpha, \beta)$,

$$
\begin{aligned}
\log \left(\frac{A+2 r}{3 r}\right) \geq \log \left(\frac{\sqrt{N s}(\log N)^{\alpha}}{3 r}\right) & \geq \log \left(\frac{\sqrt{N}(\log N)^{\alpha-(\beta / 2)}}{3 r}\right) \\
& =\frac{1}{2} \log N+\left(\alpha-\frac{\beta}{2}\right) \log \log N-\log (3 r) \geq \frac{1}{4} \log N .
\end{aligned}
$$

Plug the above bounds in (5.20) to see that for $N \geq N_{0}(t) \vee N_{1}(\alpha, \beta)$,

$$
P_{w \sqrt{N}}\left(t_{3 r}<T_{A}\right) \leq 4 C(t) \frac{\log (1 /|w|)}{\log N} .
$$

For the second term in (5.19), take $k \geq 1 / \alpha$ and use (5.5) to get

$$
\begin{aligned}
P_{w \sqrt{N}}\left(T_{A} \leq N s\right) & =P_{0}\left(\sup _{u \leq N s}\left|w \sqrt{N}+Y_{u}\right| \geq A\right) \leq P_{0}\left(\sup _{u \leq N s}\left|Y_{u}\right| \geq A-|w| \sqrt{N}\right) \\
& =P_{0}\left(\sup _{u \leq N s}\left|Y_{u}\right| \geq \sqrt{N s}(\log N)^{\alpha}\right) \leq C_{5.5}(k) \frac{(N s)^{k}}{\left(\sqrt{N s}(\log N)^{\alpha}\right)^{2 k}} \\
& \leq C(\log N)^{-2 k \alpha} \leq C(\log N)^{-2}
\end{aligned}
$$

for $N s \geq N(\log N)^{-\beta} \geq 1$. Plugging this bound and (5.21) into (5.19) we get (5.18) for $N \geq N_{2}$ depending only on $\alpha, \beta, t$. By the upper bound on $|w|$ we may increase $C_{5.18}$ to get (5.18) for all $N \geq 3$ (recall (1.13)). 


\section{The two particle dual}

In this section we collect some properties of the two-particle dual which will be needed in our analysis of the martingale square functions. Our main focus will be on the difference of the two particles. Define

$$
\psi_{r}(a)= \begin{cases}\rho^{2}\left|B_{r} \cap B_{r}(a)\right| & \text { if } a \neq 0 \\ \rho\left|B_{r}\right| & \text { if } a=0\end{cases}
$$

and observe that $\psi_{r}(a)$ is decreasing in $|a|$ and $0 \leq \psi_{r}(a) / \rho\left|B_{r}\right| \leq 1$. Consider the two-particle dual $\left(\xi_{t}^{1}, \xi_{t}^{2}\right)$ starting at $\left(x_{1}, x_{2}\right), x=x_{1}-x_{2}$, the difference process $\tilde{\xi}_{t}=$ $\tilde{\xi}_{t}^{x}=\xi_{t}^{1}-\xi_{t}^{2}$, and the coalescence time $\tau$ defined in (1.6). By the dynamics defining the two-particle dual (recall (1.5)), the fact that $\left|B_{r}(a) \cap B_{r}(b)\right|=\left|B_{r}(0) \cap B_{r}(a-b)\right|$ shows that for $y \neq 0, \tilde{\xi}$ makes transitions

$$
y \rightarrow \begin{cases}y+\bar{U} & \text { at rate } 2 \rho\left|B_{r}\right|-2 \psi_{r}(y) \\ 0 & \text { at rate } \psi_{r}(y)\end{cases}
$$

while 0 is a trap for $\tilde{\xi}$. Let

$$
\tilde{\tau}=\tilde{\tau}(x)=\inf \left\{t \geq 0: \tilde{\xi}_{t}^{x}=0\right\}(=\tau)
$$

be the time at which $\tilde{\xi}_{t}^{x}$ jumps to 0. By standard results (see Sections 4.2 and 4.4 of [19]) $\tilde{\xi}^{x}$ is a pure jump Feller process which is the unique in law solution of the martingale problem for its generator $\tilde{A}$ on the space $B\left(\mathbb{R}^{d}\right)$ of bounded Borel measurable functions. $\tilde{A}$ is given by

$$
\tilde{A} f(x)=\left(2 \rho\left|B_{r}\right|-2 \psi_{r}(x)\right) \int_{\mathbb{R}^{d}}(f(x+u)-f(x)) h_{\bar{U}}(u) d u+\psi_{r}(x)(f(0)-f(x)) .
$$

Recall from Section 5 that $Y_{t}^{x}$ is the rate $2 \rho\left|B_{r}\right|$ random walk starting at $x \in \mathbb{R}^{d}$ under $P_{x}$, and with jump distribution that of $\bar{U}$ and generator $A^{Y}$ given in (5.1) for $f \in B\left(\mathbb{R}^{d}\right)$. For a random variable $V$ we let $Y^{V}$ denote the same random walk with initial law that of $V$, and will use this notation with other Markov processes below.

We will construct a version of $\tilde{\xi}_{t}^{x}$ by absorbing a random time change of $Y^{x}$ at 0 . Define $\beta(y)=1-\frac{\psi_{r}(y)}{\rho\left|B_{r}\right|}$ and

$$
I(t)=\int_{0}^{t} \frac{1}{\beta\left(Y_{s}^{x}\right)} d s
$$

Note that for $x \neq 0$,

$$
\sup _{s \leq t} \frac{\psi_{r}\left(Y_{s}^{x}\right)}{\rho\left|B_{r}\right|}=\sup _{s \leq t} \frac{\rho\left|B_{r}\left(Y_{s}^{x}\right) \cap B_{r}\right|}{\left|B_{r}\right|}<1 \text { a.s. }
$$

and thus $\inf _{s \leq t} \beta\left(Y_{s}^{x}\right)>0$ a.s. This implies that $I(t)$ is finite and strictly increasing a.s. for all $t$. Evidently $I(t)=\infty$ for all $t>0$ if $x=0$. We will allow $x=0$ later, but until otherwise indicated we will take our initial point $x \neq 0$. From the definition of $I$ we see that for $0<s<t$,

$$
t-s \leq I(t)-I(s)
$$

Therefore $I^{-1}(t)$ exists for all $t$ a.s., and

$$
\int_{0}^{I^{-1}(t)} \frac{1}{\beta\left(Y_{s}^{x}\right)} d s=t
$$


If we define $\tilde{Y}_{t}^{x}=Y_{I^{-1}(t)}^{x}$, then it follows from (6.6) that for all but countably many $t$,

$$
\left(I^{-1}\right)^{\prime}(t)=\beta\left(Y_{I^{-1}(t)}^{x}\right)
$$

and therefore that

$$
I^{-1}(t)=\int_{0}^{t} \beta\left(\tilde{Y}_{s}^{x}\right) d s
$$

Clearly, $I^{-1}(t) \leq t$. For $x=0$ it is natural to define $I^{-1}(t)=0$ for all $t \geq 0$, which means that $\tilde{Y}_{t}^{0}:=Y_{I^{-1}(t)}^{0}=0$ for all $t \geq 0$. Thus (6.7) holds for all $x$ and

$$
\tilde{Y}_{t}^{x}=Y_{I^{-1}(t)}^{x}=Y^{x}\left(\int_{0}^{t} \beta\left(\tilde{Y}_{s}^{x}\right) d s\right) \quad \forall x \in \mathbb{R}^{d} .
$$

We may apply Theorems 1.1 and 1.3 of Sec. 6.1 of [19] to see that $\tilde{Y}^{x}$ is the unique solution of the martingale problem for

$$
A^{\tilde{Y}} f(x)=\beta(x) A^{Y} f(x)=\left(2 \rho\left|B_{r}\right|-2 \psi_{r}(x)\right) \int_{\mathbb{R}^{d}}(f(x+u)-f(x)) h_{\bar{U}}(u) d u, \quad f \in B\left(\mathbb{R}^{d}\right) .
$$

Here we note that the continuity of $f$ is not needed for Theorem 1.3 of [19] in our jump process setting as the proof there shows. Uniqueness of the martingale problem is classical for such bounded jump generators, e.g., see Theorem 4.1 in Chapter 4 of [19]), and so $\tilde{Y}^{x}$ is the unique Feller process with generator $A^{\tilde{Y}}$, and in particular is strong Markov. Finally we send $\tilde{Y}^{x}$ to its absorbing state, 0 according to the continuous additive functional

$$
C_{t}=C_{t}^{x}=\int_{0}^{t} \psi_{r}\left(\tilde{Y}_{s}^{x}\right) d s
$$

For an independent mean one exponential random variable, e, define the absorbing time

$$
\kappa=\kappa_{x}=\inf \left\{t \geq 0: C_{t}^{x}>\mathbf{e}\right\},
$$

and the absorbed process

$$
\tilde{\xi}_{t}^{x}= \begin{cases}\tilde{Y}^{x}(t) & \text { if } t<\kappa \\ 0 & \text { if } t \geq \kappa\end{cases}
$$

Then $\tilde{\xi}^{\prime} x$ is a Feller jump process and an elementary calculation shows that it solves the martingale problem for $A^{\tilde{Y}} f(x)+\psi_{r}(x)(f(0)-f(x))=\tilde{A} f(x), f \in B\left(\mathbb{R}^{d}\right)$ (from (6.3)). From (6.1) we see that the two-particle dual difference, $\tilde{\xi}$, is the Feller jump process satisfying the same well-posed martingale problem, and so, as the notation suggests, $\tilde{\xi}^{\prime}$ has the same law as $\tilde{\xi}^{x}$.

We have proved:

Lemma 6.1. If $\left(x_{1}, x_{2}\right) \in \mathbb{R}^{d} \times \mathbb{R}^{d}$, and $x=x_{1}-x_{2}$ define $I^{-1}(t)$ by (6.6) and set $\tilde{Y}^{x}(t)=Y^{x}\left(I^{-1}(t)\right)$. If

$$
\tilde{\xi}_{t}^{x}= \begin{cases}\tilde{Y}^{x}(t) & \text { if } t<\kappa, \\ 0 & \text { if } t \geq \kappa,\end{cases}
$$

where $\kappa=\kappa_{x}$ is as in (6.9), then

$$
I^{-1}(t) \int_{0}^{t} \beta\left(\tilde{Y}_{s}^{x}\right) d s
$$

and $\tilde{\xi}^{x}$ is a version of the dual difference $\xi_{t}^{1}-\xi_{t}^{2}$ under $P_{\left\{x_{1}, x_{2}\right\}}$. Moreover

$$
\tilde{\tau}(x)=\kappa_{x} \text { for all } x \neq 0 .
$$


We often denote the starting point $x$ of $\tilde{\xi}$ in the underlying probability as $P_{x}$. The tail behaviour of the coalescing time $\kappa_{x}$ will be important for us. Introduce

$$
k(a)=\rho\left|B_{r}\right| \frac{\psi_{r}(a)}{\rho\left|B_{r}\right|-\psi_{r}(a)}, \quad a \in \mathbb{R}^{d} .
$$

Lemma 6.2. If $x \in \mathbb{R}^{d} \backslash\{0\}$, then

$$
P_{x}(\kappa>t)=E_{x}\left[\exp \left(-\int_{0}^{I^{-1}(t)} k\left(Y_{s}\right) d s\right)\right]
$$

Proof. By definition of $\kappa$,

$$
P_{x}(\kappa>t)=E_{x}\left[e^{-C_{t}}\right]=E_{x}\left[\exp \left(-\int_{0}^{t} \psi_{r}\left(\tilde{Y}_{s}\right) d s\right)\right]=E_{x}\left[\exp \left(-\int_{0}^{t} \psi_{r}\left(Y_{I^{-1}(s)}\right) d s\right)\right] .
$$

Now change variables with $I(u)=s$ and use $I^{\prime}(u)=1 / \beta\left(Y_{u}\right)$ to get the required expression. to $\tilde{Y}_{t}^{x}$.

The following result shows that $I^{-1}(t)$ is close to $t$, and so $Y_{t}^{x}$ is a good approximation

Lemma 6.3. There is a constant $C_{6.3}>0$ such that for all $0<\alpha<1$ and $t>1$, and $Y_{0}=x,|x|>2 r$, or $Y_{0}=\bar{U}$,

$$
P_{Y_{0}}\left(I^{-1}(t) \notin\left[t-t^{\alpha}, t\right]\right) \leq C_{6.3} \begin{cases}\log (1+t) t^{-\alpha} & \text { if } d=2 \\ t^{-\alpha} & \text { if } d \geq 3 .\end{cases}
$$

Proof. Let $Y_{0}=x,|x|>2 r$. By (6.4) and $Y_{s} \neq 0$ for all $s$,

$$
0 \leq I(t)-t=\int_{0}^{t} \frac{\rho\left|B_{r}\right|}{\rho\left|B_{r}\right|-\psi_{r}\left(Y_{s}\right)}-1 d s=\int_{0}^{t} \frac{\rho\left|B_{r} \cap B_{r}\left(Y_{s}\right)\right|}{\left|B_{r}\right|-\rho\left|B_{r} \cap B_{r}\left(Y_{s}\right)\right|} d s .
$$

By an elementary argument, there is a constant $C_{6.15}=C_{6.15}(d, r)>0$ such that

$$
\frac{\left|B_{r} \cap B_{r}(a)\right|}{\left|B_{r}\right|-\left|B_{r} \cap B_{r}(a)\right|} \leq \frac{\left|B_{r}\right|}{\left|B_{r}\right|-\left|B_{r} \cap B_{r}(a)\right|} 1(|a| \leq 2 r) \leq C_{6.15} \frac{1}{|a|} 1\{|a| \leq 2 r\}, \quad a \in \mathbb{R}^{d} .
$$

We are assuming $|x|>2 r$, so using the density bound (5.2), we see that

$$
\begin{aligned}
E_{x}\left[\frac{1}{\left|Y_{s}\right|} 1\left\{\left|Y_{s}\right| \leq 2 r\right\}\right] & =\int_{0}^{\infty} \frac{1}{u^{2}} P_{x}\left(\left|Y_{s}\right| \leq u \wedge 2 r\right) d u \\
& =\int_{0}^{2 r} \frac{1}{u^{2}} P_{x}\left(Y_{s} \in B_{u}\right) d u+\int_{2 r}^{\infty} \frac{1}{u^{2}} P_{x}\left(Y_{s} \in B_{2 r}\right) d u \\
& \leq \frac{C_{5.2}}{s^{d / 2}+1}\left[\int_{0}^{2 r} \frac{1}{u^{2}}\left|B_{u}\right| d u+\int_{2 r}^{\infty} \frac{1}{u^{2}}\left|B_{2 r}\right| d u\right] \\
& =\frac{C(r)}{s^{d / 2}+1} .
\end{aligned}
$$

On account of (6.15), plugging this bound into (6.14) gives

$$
E_{x}(I(t)-t) \leq C_{6.15} \int_{0}^{t} \frac{C(r)}{s^{d / 2}+1} d s \leq C \begin{cases}\log (1+t) & \text { if } d=2 \\ 1 & \text { if } d \geq 3\end{cases}
$$


Applying Markov's inequality we obtain

$$
P_{x}\left(I(t)-t \geq t^{\alpha}\right) \leq C_{6.3} \begin{cases}\log (1+t) t^{-\alpha} & \text { if } d=2 \\ t^{-\alpha} & \text { if } d \geq 3\end{cases}
$$

This proves (6.13), because by (6.5), $P_{x}\left(t-I^{-1}(t) \geq t^{\alpha}\right) \leq P_{x}\left(I(t)-t \geq t^{\alpha}\right)$, and we also have $I^{-1}(t) \leq t$ by $I(t) \geq t$. The proof for $Y^{\bar{U}}$ is essentially the same.

Lemma 6.4. For $\beta \in\left(\frac{1}{4}, \frac{1}{2}\right)$ there exists a constant $C_{6.4}=C_{6.4}(\beta)>0$ such that

$$
P\left(\left|\tilde{Y}_{t}^{\bar{U}}\right| \leq r_{0}\right) \leq C_{6.4} t^{2 \beta-1} \quad \text { for all } t>0, r_{0} \leq t^{\beta} .
$$

Proof. The bounds on $\beta$ imply that $0<1-2 \beta<2 \beta<1$. This means we can choose $\alpha \in(0,1)$ such that $1-2 \beta<\alpha<2 \beta$. For this $\alpha$ choose $t$ large enough so that $\log (1+t) \geq 1$ and $t-t^{\alpha}>t / 2$. By (6.13),

$$
\begin{aligned}
P\left(\left|\tilde{Y}_{t}^{\bar{U}}\right| \leq r_{0}\right)= & P\left(\left|Y_{I^{-1}(t)}^{\bar{U}}\right| \leq r_{0}\right) \\
\leq & P\left(I^{-1}(t) \notin\left[t-t^{\alpha}, t\right]\right)+P\left(\inf _{s \in\left[t-t^{\alpha}, t\right]}\left|Y_{s}^{\bar{U}}\right| \leq r_{0}\right) \\
\leq & C_{6.3} \log (1+t) t^{-\alpha}+P\left(\left|Y_{t-t^{\alpha}}^{\bar{U}}\right| \leq r_{0}+t^{\beta}\right)+ \\
& \quad+P\left(\left|Y_{t-t^{\alpha}}^{\bar{U}}\right|>r_{0}+t^{\beta}, \inf _{s \in\left[t-t^{\alpha}, t\right]}\left|Y_{s}^{\bar{U}}\right| \leq r_{0}\right) .
\end{aligned}
$$

By $r_{0} \leq t^{\beta},(5.2)$, and the above choice of $t$,

$$
P\left(\left|Y_{t-t^{\alpha}}^{\bar{U}}\right| \leq r_{0}+t^{\beta}\right) \leq C \frac{\left(r_{0}+t^{\beta}\right)^{d}}{\left(t-t^{\alpha}\right)^{d / 2}} \leq C \frac{\left(2 t^{\beta}\right)^{d}}{(t / 2)^{d / 2}} \leq C t^{\frac{d}{2}(2 \beta-1)} \leq C t^{2 \beta-1}
$$

For the last term in (6.17), the Markov property and (5.5) imply that (recall $t>1$ ) for all $k \geq 1$,

$$
P\left(\left|Y_{t-t^{\alpha}}^{\bar{U}}\right|>r_{0}+t^{\beta}, \inf _{s \in\left[t-t^{\alpha}, t\right]}\left|Y_{s}^{\bar{U}}\right| \leq r_{0}\right) \leq P\left(\sup _{s \leq t^{\alpha}}\left|Y_{s}^{0}\right| \geq t^{\beta}\right) \leq C_{5.5}(k) t^{k(\alpha-2 \beta)} .
$$

Plugging these bounds into (6.17) gives

$$
P\left(\left|\tilde{Y}_{t}^{\bar{U}}\right| \leq r_{0}\right) \leq C\left(t^{-\alpha} \log (1+t)+t^{2 \beta-1}+t^{k(\alpha-2 \beta)}\right) .
$$

Recalling that $\alpha>1-2 \beta$ and $\alpha-2 \beta<0$, and choosing $k$ large such that $k(\alpha-2 \beta)<2 \beta-1$, it follows that for large $t, P\left(\left|\tilde{Y}_{t}^{\bar{U}}\right| \leq r_{0}\right) \leq C t^{2 \beta-1}$. Increasing $C$ appropriately to handle small $t$ completes the proof.

For $0<a<b$ define

$$
G(a, b)=\left\{\left|Y_{I^{-1}(u)}\right|>2 r \forall u \in[a, b]\right\},
$$

and for $q \geq 1$ introduce

$$
s_{N}=(\log N)^{-q} .
$$

Lemma 6.5. There is a constant $C_{6.20}(q)>0$ such that

$$
P_{x}\left(G\left(\frac{N s_{N}}{4}, 2 N s_{N}\right)^{c}\right) \leq C_{6.20} \frac{\log \log N}{\log N} \quad \text { for all }|x|>2 r .
$$


Proof. Recall from (1.13) that $N \geq 3$. Let $u_{N}=N s_{N} / 4$ and $u_{N}^{\prime}=u_{N}-u_{N}^{1 / 2}$. Then since $I^{-1}(u) \leq u$,

$$
P_{x}\left(G\left(u_{N}, 2 N s_{N}\right)^{c}\right) \leq P_{x}\left(I^{-1}\left(u_{N}\right) \leq u_{N}^{\prime}\right)+P_{x}\left(\left|Y_{u}\right| \leq 2 r \text { for some } u \in\left[u_{N}^{\prime}, 2 N s_{N}\right]\right) .
$$

By (6.13) with $\alpha=1 / 2$ and $N \geq N_{0}(q)$ (recall $|x|>2 r$ ),

$$
P_{x}\left(I^{-1}\left(u_{N}\right) \leq u_{N}^{\prime}\right) \leq C \frac{\log \left(1+u_{N}\right)}{\sqrt{u_{N}}} \leq \frac{C}{N^{1 / 4}} .
$$

Next, using the Markov property at time $u_{N}^{\prime}$, we have for $N \geq N_{0}(q)$,

$$
\begin{aligned}
& P_{x}\left(\left|Y_{u}\right| \leq 2 r \text { for some } u \in\left[u_{N}^{\prime}, 2 N s_{N}\right]\right) \\
& \leq P_{x}\left(\left|Y_{u_{N}^{\prime}}\right| \leq s_{N} \sqrt{N}\right)+\sup _{|y| \geq s_{N}} P_{y \sqrt{N}}\left(t_{2 r} \leq 2 N s_{N}-u_{N}^{\prime}\right) \\
& \leq \exp \left(-2 \rho\left|B_{r}\right| u_{N}^{\prime}\right)+C \frac{\left(s_{N} \sqrt{N}\right)^{d}}{\left(u_{N}^{\prime}\right)^{d / 2}}+C \frac{\log \left(1 / s_{N}\right)}{\log N} \\
& \quad \quad(\text { by (5.2), (5.18) (if } d=2) \text { and (5.8) (if } d=3)) \\
& \leq \frac{C}{\log N}+C \frac{\log \log N}{\log N} .
\end{aligned}
$$

In the next to last line we have used the $d=2$ bound; if $d \geq 3$, (5.8) gives a much smaller bound. Here we have also used the strong Markov property and applied (5.18) (if $d=2$ ) with $w \sqrt{N}$ equal to the location of the first jump into $B_{s_{N} \sqrt{N}}$. Use this bound and (6.22) in (6.21) derive (6.20) for $N \geq N_{0}(q)$. Now adjust $C_{6.20}$ to handle the remaining values of $N$.

We will also need a bound on the two-particle dual $\xi_{t}=\left(\xi^{1}, \xi_{t}^{2}\right)$ after the coalescing time $\kappa$ for any $d \geq 2$. In this setting assume

$$
W^{1, x_{1}}, W^{2, x_{2}} \text { and } W^{3,0} \text { are independent rate } \rho\left|B_{r}\right| \text { random walks in } \mathbb{R}^{d} \text { with step }
$$

distribution $\bar{U}$ (now in $\mathbb{R}^{d}$ ) and starting at points $x_{1}, x_{2}, 0 \in \mathbb{R}^{d}$, respectively.

Define $W_{t}=\left(W_{t}^{1, x_{1}}, W_{t}^{2, x_{2}}\right)$, and

$$
\psi_{W}\left(y_{1}, y_{2}\right)=k\left(\left|y_{1}-y_{2}\right|\right) 1\left(y_{1} \neq y_{2}\right)=\frac{\rho\left|B_{r}\right| \psi_{r}\left(y_{1}-y_{2}\right)}{\rho\left|B_{r}\right|-\psi_{r}\left(y_{1}-y_{2}\right)} 1\left(y_{1} \neq y_{2}\right),
$$

and $D(t)=\int_{0}^{t} \psi_{W}\left(W_{s}\right) d s$. Although $\psi_{W}\left(y_{1}-y_{2}\right)$ becomes unbounded (if $\rho=1$ ) as $y_{1}-y_{2} \rightarrow 0$, as for $I(t), D(t)<\infty$ for all $t>0$ a.s. Let $\mathbf{e}$ be an independent exponential mean 1 random variable, and introduce $\bar{\kappa}=\inf \{t \geq 0: D(t)>\mathbf{e}\} \leq \infty$ (it will be a.s. finite if $d=2$ ). Assume also that conditional on $\left(W, W^{3,0}, \mathbf{e}\right), U_{W(\bar{\kappa})}$ has a uniform distribution on $B_{r}\left(W_{\bar{\kappa}-}^{1, x_{1}}\right) \cap B_{r}\left(W_{\bar{\kappa}-}^{2, x_{2}}\right)$ (the intersection is non-empty a.s. by the definition of $\bar{\kappa}$ because $\psi_{W}\left(y_{1}, y_{2}\right)=0$ if $\left|y_{1}-y_{2}\right|>2 r$ ), and given $\left(W, W^{3,0}, \mathbf{e}, U_{W(\bar{\kappa})}\right), U$ is an independent r.v. uniformly distributed on $B_{r}$. We can use the above to define a version of our two-particle dual but now "run at a constant rate" by

$$
\bar{W}_{t}= \begin{cases}\left(W_{t}^{1, x_{1}}, W_{t}^{1, x_{1}}\right) & \text { if } x_{1}=x_{2}, \\ W_{t} & \text { if } t<\bar{\kappa} \text { and } x_{1} \neq x_{2}, \\ \left(U+U_{W_{\bar{\kappa}}}+W_{t-\bar{\kappa}}^{3,0}, U+U_{W_{\bar{\kappa}}}+W_{t-\bar{\kappa}}^{3,0}\right) & \text { if } t \geq \bar{\kappa} \text { and } x_{1} \neq x_{2} .\end{cases}
$$


Note that when $\bar{W}$ is at $\left(y_{1}, y_{2}\right), y_{1} \neq y_{2}, \bar{W}$ jumps to the diagonal in $\mathbb{R}^{d} \times \mathbb{R}^{d}$ at rate $\psi_{W}\left(y_{1}, y_{2}\right)$.

We now extend our earlier time change and define

$$
\begin{aligned}
\beta\left(y_{1}, y_{2}\right) & =\left[1-\frac{\psi_{r}\left(y_{1}-y_{2}\right)}{\rho\left|B_{r}\right|}\right] 1\left(y_{1} \neq y_{2}\right)+1\left(y_{1}=y_{2}\right) \leq 1, \quad \forall y \in \mathbb{R}^{d}, \\
\bar{I}(t) & =\int_{0}^{t} \beta\left(\overline{W_{s}}\right)^{-1} d s<\infty \quad \forall t>0, \\
\overline{\xi_{t}} & =\bar{W}\left(\bar{I}^{-1}(t)\right),
\end{aligned}
$$

where $\bar{I}^{-1}$ is the inverse of the strictly increasing continuous function $\bar{I}$. As in (6.7), one sees that

$$
\bar{I}^{-1}(t)=\int_{0}^{t} \beta\left(\bar{\xi}_{s}\right) d s \quad \forall t \geq 0 .
$$

The following result shows that $\bar{\xi}_{t}$ is a version of the two particle dual.

Lemma 6.6. If $\left(x_{1}, x_{2}\right) \in \mathbb{R}^{d} \times \mathbb{R}^{d}$ define $\bar{W}$ by (6.24), $\beta$ by (6.25), $\bar{I}$ by (6.26), and $\bar{\xi}$ by (6.27). Then

$$
\bar{I}^{-1}(t)=\int_{0}^{t} \beta\left(\bar{\xi}_{s}\right) d s
$$

and $\bar{\xi}$ is a version of the two-particle dual described in (1.5) under $P_{\left\{x_{1}, x_{2}\right\}}$.

Proof. The jump rate of $\bar{W}$ to the diagonal becomes unbounded as it approaches the diagonal (for $\rho=1$ ), so we proceed more carefully than in the proof of Lemma 6.1, making use of optional stopping. Let

$$
R_{n}=\left\{\left(y_{1}, y_{2}\right) \in \mathbb{R}^{d} \times \mathbb{R}^{d}: 0<\left|y_{1}-y_{2}\right|<n^{-1}\right\}
$$

and

$$
T_{n}^{W}=\inf \left\{t \geq 0: \bar{W}_{t} \in R_{n}\right\} \leq \infty .
$$

Then $\overline{W_{n}}(t)=\bar{W}\left(t \wedge T_{n}^{W}\right)$ is a pure jump process on $\mathbb{R}^{d} \times \mathbb{R}^{d}$ with bounded jump rates and generator

$$
\begin{aligned}
\bar{A}_{n} f(y)= & \rho\left|B_{r}\right|\left[E\left(f\left(y_{1}+\bar{U}, y_{2}\right)+f\left(y_{1}, y_{2}+\bar{U}\right)-2 f(y)\right)\right] 1\left(\left|y_{1}-y_{2}\right| \geq 1 / n\right) \\
& +\psi_{W}(y)\left[E\left(f\left(U+U_{y}, U+U_{y}\right)-f(y)\right)\right] 1\left(\left|y_{1}-y_{2}\right| \geq 1 / n\right) \\
& +\rho\left|B_{r}\right|[E(f(y+(\bar{U}, \bar{U}))-f(y))] 1\left(y_{1}=y_{2}\right) .
\end{aligned}
$$

Here $U_{y}$ is uniformly distributed on $B_{r}\left(y_{1}\right) \cap B_{r}\left(y_{2}\right)$ and is independent of the uniform (on $B_{r}$ ) r.v. $U$. It is easy to check that $\bar{W}_{n}$ solves the martingale problem for $\bar{A}_{n}$ on the domain $B\left(\mathbb{R}^{d} \times \mathbb{R}^{d}\right)$ of bounded Borel functions on $\mathbb{R}^{d} \times \mathbb{R}^{d}$.

Let $\bar{T}_{n}=\inf \left\{t \geq 0: \bar{\xi}_{t} \in R_{n}\right\} \leq \infty$. Using the properties of $\bar{I}^{-1}$ and (6.28), it is easy to check that

$$
T_{n}^{W}=\bar{I}^{-1}\left(\bar{T}_{n}\right)=\int_{0}^{\bar{T}_{n}} \beta\left(\bar{\xi}_{s}\right) d s
$$

It follows that

$$
I^{-1}\left(t \wedge \bar{T}_{n}\right)=I^{-1}(t) \wedge T_{n}^{W}=\int_{0}^{t} \beta\left(\bar{\xi}_{s}\right) d s \wedge T_{n}^{W}=\int_{0}^{t} \beta\left(\bar{\xi}\left(s \wedge \bar{T}_{n}\right)\right) d s \wedge T_{n}^{W} .
$$

If we define $\bar{\xi}_{t}^{\bar{T}_{n}}=\bar{\xi}\left(t \wedge \bar{T}_{n}\right)$, the above implies $\bar{\xi}_{t}^{\bar{T}_{n}}=\bar{W}_{n}\left(\int_{0}^{t} \beta\left(\bar{\xi}_{s}^{\bar{T}_{n}}\right)\right) d s$, and thus we may apply Theorems 1.1 and 1.3 in Chapter 6 of [19] to conclude that $\bar{\xi}_{t}^{\bar{T}_{n}}$ solves the martingale problem for

$$
G_{n} f(y)=\beta(y) \bar{A}_{n} f(y), \quad f \in B\left(\mathbb{R}^{d} \times \mathbb{R}^{d}\right) .
$$


Here we recall again that the continuity of $f$ assumed in Ch. 6 Theorem 1.3 of [19] is not needed in our jump process setting. A bit of arithmetic shows

$$
\begin{aligned}
G_{n} f(y)=( & \left.\rho\left|B_{r}\right|-\psi_{r}\left(y_{1}-y_{2}\right)\right)\left[E\left(f\left(y_{1}+\bar{U}, y_{2}\right)+f\left(y_{1}, y_{2}+\bar{U}\right)-2 f(y)\right)\right] 1\left(\mid y_{1}-y_{2} \geq 1 / n\right) \\
& +\psi_{r}\left(y_{1}-y_{2}\right)\left[E\left(f\left(U+U_{y}, U+U_{y}\right)-f(y)\right)\right] 1\left(\mid y_{1}-y_{2} \geq 1 / n\right) \\
& +\rho\left|B_{r}\right|[E(f(y+(\bar{U}, \bar{U}))-f(y))] 1\left(y_{1}=y_{2}\right) .
\end{aligned}
$$

If $\xi=\left(\xi^{1}, \xi^{2}\right)$ is the two-particle dual process, as described in (1.5), the above is the generator of the Feller pure jump process $\xi\left(t \wedge T_{n}\right)$, where $T_{n}=\inf \left\{t \geq 0: \xi_{t} \in R_{n}\right\}$ and so $\xi^{T_{n}}(t)=\xi\left(t \wedge T_{n}\right)$ also solves the martingale problem for $G_{n}\left(f \in B\left(\mathbb{R}^{d} \times \mathbb{R}^{d}\right)\right)$. By well-posedness of this martingale problem ((Section 2 and Thm. 4.1 of Chapter 4 of [19]) we concluded that $\xi^{T_{n}}$ and $\bar{\xi}^{\bar{T}_{n}}$ are identical in law for all $n \in \mathbb{N}$. Since $R_{n} \downarrow \emptyset$ and $\xi\left(T_{n}\right), \bar{\xi}\left(\bar{T}_{n}\right) \in R_{n}$ when these times are finite, it follows that $T_{n}, \bar{T}_{n} \uparrow \infty$ a.s. as $n \rightarrow \infty$ (in fact for large $n$ they will be infinite a.s.), and therefore $\xi$ and $\bar{\xi}$ are identical in law.

The following result is now an easy consequence of (6.24), Lemma 6.6 and the bound $\bar{I}^{-1}(t) \leq t$ for all $t \geq 0$.

Lemma 6.7. Assume $\xi^{x}$ is the two-particle dual in $\mathbb{R}^{d} \times \mathbb{R}^{d}$, starting at $x=\left(x_{1}, x_{2}\right)$. Then we may assume there are random walks $W^{i, x_{i}}\left(i=0,1,2, x_{0}=0\right)$ as in (6.23) such that

$$
\sup _{s \leq t}\left|\xi_{s}^{x}\right| \leq\left[\sum_{i=0}^{3} \sup _{s \leq t}\left|W_{s}^{i, x_{i}}\right|\right]+2 \sqrt{2} r
$$

\section{Analysis of the square function: Proof of Proposition 4.1}

In this section we analyze the martingale square function $\left\langle M^{N}(\phi)\right\rangle_{t}$ for $\phi \in C_{0}^{3}\left(\mathbb{R}^{d}\right)$, and in particular give the Proof of Proposition 4.1. We recall from Proposition 2.2 and Lemma 2.4 that

$$
\left\langle M^{N}(\phi)\right\rangle_{t}=\int_{0}^{t} m_{s}^{N}(\phi) d s=\int_{0}^{t}\left(\bar{m}_{s}^{N}(\phi)+\mathcal{E}_{2.10}^{N}(\phi, s)\right) d s,
$$

and that the integral of $\mathcal{E}_{2.10}^{N}(\phi, s)$ is negligible for all $d \geq 2$ on account of (2.10). We can thus focus on $\bar{m}_{s}^{N}(\phi)$ defined in (2.9), which we write in the form

$$
\bar{m}_{s}^{N}(\phi)=\bar{m}_{s}^{N, 1}(\phi)-\bar{m}_{s}^{N, 2}(\phi),
$$

where

$$
\begin{aligned}
& \bar{m}_{s}^{N, 1}(\phi)=\rho^{2} N^{1+d / 2}\left|B_{r}^{N}\right| K^{2} \int_{\mathbb{R}^{d}} \phi^{2}(x) d x \int_{B_{r}^{N}(x)} w_{s}^{N}(z) d z \\
& \bar{m}_{s}^{N, 2}(\phi)=\rho^{2} N^{1+d / 2} K^{2} \int_{\mathbb{R}^{d}} \phi^{2}(x) d x \int_{B_{r}^{N}(x)} \int_{B_{r}^{N}(x)} w_{s}^{N}\left(z_{1}\right) w_{s}^{N}\left(z_{2}\right) d z_{1} d z_{2} .
\end{aligned}
$$

Recall that $K=K^{\prime} N^{d / 2-1}$ where $K^{\prime}=1$ if $d \geq 3$ and $K^{\prime}=\log N$ if $d=2$, and define

$$
\bar{\gamma}^{N}(s)=K^{\prime} \gamma_{e}(N s)
$$

Lemma 7.1. There is a constant $C_{7.4}=C_{7.4}(\phi)>0$ such that for $s \geq 0$,

$$
E\left(\bar{m}_{s}^{N, 1}(\phi)\right)=\rho^{2}\left|B_{r}\right|^{2} K^{\prime} X_{0}^{N}\left(\phi^{2}\right)+\mathcal{E}_{7.4}(\phi, s)
$$

where

$$
\left|\mathcal{E}_{7.4}(\phi, s)\right| \leq C_{7.4} K^{\prime}\left(\frac{1}{\sqrt{N}}+\sqrt{s}\right) X_{0}^{N}(\mathbf{1})
$$


Proof. By a change of variables,

$$
\begin{aligned}
\int_{\mathbb{R}^{d}} \phi^{2}(x) \int_{B_{r}^{N}(x)} w_{s}^{N}(z) d z d x & =\int_{\mathbb{R}^{d}} w_{s}^{N}(z) \int_{B_{r}^{N}(z)} \phi^{2}(x) d x d z \\
& =\int_{\mathbb{R}^{d}} w_{s}^{N}(z) \int_{B_{r}^{N}(z)}\left[\phi^{2}(z)+\left(\phi^{2}(x)-\phi^{2}(z)\right)\right] d x d z \\
& =\left|B_{r}^{N}\right| \int_{\mathbb{R}^{d}} \phi^{2}(z) w_{s}^{N}(z) d z+\varepsilon_{s}^{N},
\end{aligned}
$$

where we have set $\varepsilon_{s}^{N}=\int_{\mathbb{R}^{d}} \int_{B_{r}^{N}(x)} w_{s}^{N}(z)\left[\phi^{2}(x)-\phi^{2}(z)\right] d x d z$. For $z \in B_{r}^{N}(x)$ and $C_{\phi}=2\|\phi\|_{\infty}\|\phi\|_{\text {Lip }},\left|\phi^{2}(x)-\phi^{2}(z)\right| \leq C_{\phi} 2 r / \sqrt{N}$. Thus

$$
\left|\varepsilon_{s}^{N}\right| \leq C_{\phi} \frac{2 r}{\sqrt{N}} \int_{\mathbb{R}^{d}} \int_{B_{r}^{N}(x)} w_{s}^{N}(z) d x d z=C_{\phi} \frac{2 r}{\sqrt{N}}\left|B_{r}^{N}\right| w_{s}^{N}(\mathbf{1}) .
$$

Returning to the definition of $\bar{m}_{s}^{N, 1}(\phi)$ we see that

$$
E\left(\bar{m}_{s}^{N, 1}(\phi)\right)=\rho^{2} N^{1+d / 2}\left|B_{r}^{N}\right| K^{2}\left(\left|B_{r}^{N}\right| E\left(w_{s}^{N}\left(\phi^{2}\right)\right)+E\left(\varepsilon_{s}^{N}\right)\right)=\rho^{2}\left|B_{r}\right|^{2} K^{\prime} E\left(X_{s}^{N}\left(\phi^{2}\right)\right)+\mathcal{E},
$$

with

$$
|\mathcal{E}|=\rho^{2} N^{1+d / 2}\left|B_{r}^{N}\right| K^{2} E\left(\left|\varepsilon_{s}^{N}\right|\right) \leq C_{\phi} \rho^{2}\left|B_{r}\right|^{2} \frac{2 r K^{\prime}}{\sqrt{N}} E\left(X_{s}^{N}(\mathbf{1})\right)=\frac{C K^{\prime}}{\sqrt{N}} X_{0}^{N}(\mathbf{1})
$$

by the martingale property of $X_{s}^{N}(\mathbf{1})$ (Corollary 3.2).

Next, we bound the difference $\left|E\left(X_{s}^{N}\left(\phi^{2}\right)\right)-X_{0}^{N}\left(\phi^{2}\right)\right|$. By the single particle duality equation (3.2) and a change of variables,

$$
\begin{aligned}
E\left(X_{s}^{N}\left(\phi^{2}\right)\right) & =\int_{\mathbb{R}^{d}} \phi^{2}(x) E_{\{x\}}^{N}\left(X_{0}^{N}\left(\eta_{s}^{N}\right)\right) d x=\int_{\mathbb{R}^{d}} \phi^{2}(x) E_{\{0\}}^{N}\left(X_{0}^{N}\left(x+\eta_{s}^{N}\right)\right) d x \\
& =\int_{\mathbb{R}^{d}} E_{\{0\}}^{N}\left(\phi^{2}\left(x^{\prime}-\eta_{s}^{N}\right)\right) X_{0}^{N}\left(x^{\prime}\right) d x^{\prime} \\
& =X_{0}^{N}\left(\phi^{2}\right)+\int_{\mathbb{R}^{d}} E_{\{0\}}^{N}\left[\phi^{2}\left(x^{\prime}-\eta_{s}^{N}\right)-\phi^{2}\left(x^{\prime}\right)\right] X_{0}^{N}\left(x^{\prime}\right) d x^{\prime} .
\end{aligned}
$$

Using the smoothness of $\phi$ and scaling, we see that Lemma 5.1(b) (it applies to the rate $\rho\left|B_{r}\right|$ walk $\eta$ as well) implies

$$
\int_{\mathbb{R}^{d}} E_{\{0\}}^{N}\left[\left|\phi^{2}\left(x^{\prime}-\eta_{s}^{N}\right)-\phi^{2}\left(x^{\prime}\right)\right|\right] X_{0}^{N}\left(x^{\prime}\right) d x^{\prime} \leq C_{\phi} E_{\{0\}}\left(\frac{\left|\eta_{N s}\right|}{\sqrt{N}}\right) X_{0}^{N}(\mathbf{1}) \leq C_{\phi} C \sqrt{s} X_{0}^{N}(\mathbf{1}) .
$$

Combining this bound with (7.5) and (7.6) gives (7.4).

To handle $\bar{m}_{s}^{N, 2}(\phi)$ we apply the two-particle duality equation (3.3) and then split the resulting expression into two pieces, obtaining

$$
E\left(\bar{m}_{s}^{N, 2}(\phi)\right)=J_{s}^{N, 1}(\phi)+J_{s}^{N, 2}(\phi)
$$

with

$$
\begin{aligned}
J_{s}^{N, 1}(\phi)= & \rho^{2} N^{1+d / 2} K^{2} \int_{\mathbb{R}^{d}} \phi^{2}(x) \int_{B_{r}^{N}(x)} \int_{B_{r}^{N}(x)} E_{\left\{z_{1}, z_{2}\right\}}^{N}\left[w_{0}^{N}\left(\xi_{s}^{N, 1}\right)\right. \\
& \left.\times 1\left\{\tau^{N} \leq s\right\}\right] d z_{1} d z_{2} d x \\
J_{s}^{N, 2}(\phi)= & \rho^{2} N^{1+d / 2} K^{2} \int_{\mathbb{R}^{d}} \phi^{2}(x) \int_{B_{r}^{N}(x)} \int_{B_{r}^{N}(x)} E_{\left\{z_{1}, z_{2}\right\}}^{N}\left[\left(w_{0}^{N}\left(\xi_{s}^{N, 1}\right)\right) w_{0}^{N}\left(\xi_{s}^{N, 2}\right)\right. \\
& \left.1\left\{\tau^{N}>s\right\}\right] d z_{1} d z_{2} d x .
\end{aligned}
$$


Lemma 7.2. There is a constant $C_{7.10}=C_{7.10}(\phi)>0$ such that for $s \geq 0$,

$$
\begin{aligned}
J_{s}^{N, 1}(\phi) & =\rho^{2}\left|B_{r}\right|^{2}\left(K^{\prime}-\bar{\gamma}^{N}(s)\right) X_{0}^{N}\left(\phi^{2}\right)+\mathcal{E}_{7.10}, \text { where } \\
\left|\mathcal{E}_{7.10}\right| & \leq C_{7.10} K^{\prime}\left(\frac{1}{\sqrt{N}}+\sqrt{s}\right) X_{0}^{N}(\mathbf{1}) .
\end{aligned}
$$

Proof. By translation invariance, changing of variables and order of integration, we see

$$
\begin{aligned}
\int_{\mathbb{R}^{d}} & \int_{B_{r}^{N}(x)} \int_{B_{r}^{N}(x)} \phi^{2}(x) E_{\left\{z_{1}, z_{2}\right\}}^{N}\left[w_{0}^{N}\left(\xi_{s}^{N, 1}\right) 1\left\{\tau^{N} \leq s\right\}\right] d z_{1} d z_{2} d x \\
& =\int_{\mathbb{R}^{d}} \int_{B_{r}^{N}} \int_{B_{r}^{N}} \phi^{2}(x) E_{\left\{x+z_{1}^{\prime}, x+z_{2}^{\prime}\right\}}^{N}\left[w_{0}^{N}\left(\xi_{s}^{N, 1}\right) 1\left\{\tau^{N} \leq s\right\}\right] d z_{1}^{\prime} d z_{2}^{\prime} d x \\
& =\int_{B_{r}^{N}} \int_{B_{r}^{N}} E_{\left\{z_{1}^{\prime}, z_{2}^{\prime}\right\}}^{N}\left[\int_{\mathbb{R}^{d}} \phi^{2}(x) w_{0}^{N}\left(x+\xi_{s}^{N, 1}\right) 1\left\{\tau^{N} \leq s\right\} d x\right] d z_{1}^{\prime} d z_{2}^{\prime}
\end{aligned}
$$

Changing variables again with $x^{\prime}=x+\xi_{s}^{N, 1}$ and adding and subtracting $\phi^{2}\left(x^{\prime}\right)$, the right-side above equals

$$
\begin{aligned}
& \int_{B_{r}^{N}} \int_{B_{r}^{N}} E_{\left\{z_{1}^{\prime}, z_{2}^{\prime}\right\}}^{N}\left[\int_{\mathbb{R}^{d}} \phi^{2}\left(x^{\prime}-\xi_{s}^{N, 1}\right) w_{0}^{N}\left(x^{\prime}\right) 1\left\{\tau^{N} \leq s\right\} d x^{\prime}\right] d z_{1}^{\prime} d z_{2}^{\prime} \\
& =\int_{B_{r}^{N}} \int_{B_{r}^{N}} \int_{\mathbb{R}^{d}} \phi^{2}\left(x^{\prime}\right) w_{0}^{N}\left(x^{\prime}\right) P_{\left\{z_{1}^{\prime}, z_{2}^{\prime}\right\}}^{N}\left(\tau^{N} \leq s\right\} d x^{\prime} d z_{1}^{\prime} d z_{2}^{\prime} \\
& \quad+\int_{B_{r}^{N}} \int_{B_{r}^{N}} E_{\left\{z_{1}^{\prime}, z_{2}^{\prime}\right\}}^{N}\left[\int_{\mathbb{R}^{d}}\left(\phi^{2}\left(x^{\prime}-\xi_{s}^{N, 1}\right)-\phi^{2}\left(x^{\prime}\right)\right) 1\left\{\tau^{N} \leq s\right\} w_{0}^{N}\left(x^{\prime}\right) d x^{\prime}\right] d z_{1}^{\prime} d z_{2}^{\prime} \\
& =w_{0}^{N}\left(\phi^{2}\right) \int_{B_{r}^{N}} \int_{B_{r}^{N}} P_{\left\{z_{1}^{\prime}, z_{2}^{\prime}\right\}}^{N}\left(\tau^{N} \leq s\right\} d z_{1}^{\prime} d z_{2}^{\prime}+\varepsilon_{s}^{N} \\
& =w_{0}^{N}\left(\phi^{2}\right) N^{-d} \int_{B_{r}} \int_{B_{r}} P_{\left\{z_{1}, z_{2}\right\}}(\tau \leq N s\} d z_{1} d z_{2}+\varepsilon_{s}^{N} \\
& =\left|B_{r}\right|^{2} N^{-d} w_{0}^{N}\left(\phi^{2}\right)\left(1-\gamma_{e}(N s)\right)+\varepsilon_{s}^{N},
\end{aligned}
$$

where

$$
\begin{aligned}
\left|\varepsilon_{s}^{N}\right| & =\left|\int_{B_{r}^{N}} \int_{B_{r}^{N}} \int_{\mathbb{R}^{d}} E_{\left\{z_{1}^{\prime}, z_{2}^{\prime}\right\}}^{N}\left[\left(\phi^{2}\left(x^{\prime}-\xi_{s}^{N, 1}\right)-\phi^{2}\left(x^{\prime}\right)\right) 1\left\{\tau^{N} \leq s\right\}\right] w_{0}^{N}\left(x^{\prime}\right) d x^{\prime} d z_{1}^{\prime} d z_{2}^{\prime}\right| \\
& \leq C_{\phi} \int_{B_{r}^{N}} \int_{B_{r}^{N}} \int_{\mathbb{R}^{d}} E_{\left\{z_{1}^{\prime}, z_{2}^{\prime}\right\}}^{N}\left[\left|\xi_{s}^{N, 1}\right| 1\left\{\tau^{N} \leq s\right\}\right] w_{0}^{N}\left(x^{\prime}\right) d x^{\prime} d z_{1}^{\prime} d z_{2}^{\prime} \\
& =C_{\phi} w_{0}^{N}(\mathbf{1}) \int_{B_{r}^{N}} \int_{B_{r}^{N}} E_{\left\{z_{1}^{\prime}, z_{2}^{\prime}\right\}}^{N}\left(\left|\xi_{s}^{N, 1}\right| 1\left\{\tau^{N} \leq s\right\}\right) d z_{1}^{\prime} d z_{2}^{\prime} \\
& =C_{\phi} w_{0}^{N}(\mathbf{1}) N^{-d} \frac{1}{\sqrt{N}} \int_{B_{r}} \int_{B_{r}} E_{\left\{z_{1}, z_{2}\right\}}\left(\left|\xi_{N s}^{1}\right| 1\{\tau \leq N s\}\right) d z_{1} d z_{2} .
\end{aligned}
$$

For fixed $z_{1}, z_{2} \in B_{r}$, letting $z_{3}=0$, Lemma 6.7 implies that

$E_{\left\{z_{1}, z_{2}\right\}}\left(\left|\xi_{N s}^{1}\right|\right) \leq 2 \sqrt{2} r+\sum_{i=1}^{3} E\left[\sup _{t \leq N s}\left|W_{t}^{i, z_{i}}\right|\right] \leq 2 \sqrt{2} r+3\left(r+E\left(\sup _{t \leq N s}\left|Y_{t}^{0}\right|\right)\right) \leq C_{r}+C \sqrt{N s}$ using Lemma 5.1(b) for the last inequality. Plugging this bound into (7.11), we obtain

$$
\left|\varepsilon_{s}^{N}\right| \leq C\left(\frac{1}{\sqrt{N}}+\sqrt{s}\right) N^{-d} w_{0}^{N}(\mathbf{1})
$$


Returning to $J_{s}^{N, 1}$, we now have

$$
\begin{aligned}
J_{s}^{N, 1}(\phi) & =\rho^{2} N^{1+\frac{d}{2}} K^{2}\left(\left|B_{r}\right|^{2} N^{-d} w_{0}^{N}\left(\phi^{2}\right)\left(1-\gamma_{e}(N s)\right)+\varepsilon_{s}^{N}\right) \\
& =\rho^{2}\left|B_{r}\right|^{2} K^{\prime} X_{0}^{N}\left(\phi^{2}\right)\left(1-\gamma_{e}(N s)\right)+\mathcal{E} \\
& =\rho^{2}\left|B_{r}\right|^{2} X_{0}^{N}\left(\phi^{2}\right)\left[K^{\prime}-\bar{\gamma}^{N}(s)\right]+\mathcal{E},
\end{aligned}
$$

where

$$
\begin{aligned}
|\mathcal{E}| & \leq C N^{1+\frac{d}{2}} K^{2}\left|\varepsilon_{s}^{N}\right| \leq C N^{1+\frac{d}{2}} K N^{-d}\left(\frac{1}{\sqrt{N}}+\sqrt{s}\right) X_{0}^{N}(\mathbf{1}) \\
& =C K^{\prime}\left(\frac{1}{\sqrt{N}}+\sqrt{s}\right) X_{0}^{N}(\mathbf{1})
\end{aligned}
$$

which proves (7.10).

Using Lemmas 7.1 and 7.2 in (7.1) and (7.7), we arrive at the following:

Corollary 7.3. For $s \geq 0$,

$$
\left.\left|E\left(\bar{m}_{s}^{N}(\phi)\right)-\rho^{2}\right| B_{r}\right|^{2} \bar{\gamma}^{N}(s) X_{0}^{N}\left(\phi^{2}\right) \mid \leq J_{s}^{N, 2}(\phi)+C_{7.12} K^{\prime}\left(\frac{1}{\sqrt{N}}+\sqrt{s}\right) X_{0}^{N}(\mathbf{1}) .
$$

We turn now to the analysis of $J_{s}^{N, 2}(\phi) \leq\|\phi\|_{\infty}^{2} J_{s}^{N, 2}(\mathbf{1})$. Recall $Y_{t}^{x}$ and $\tilde{Y}_{t}^{x}=Y^{x}\left(I^{-1}(t)\right)$ from Section 6, $\tilde{\tau}$ from (6.2), and the process $\tilde{\xi}_{t}^{x}=\tilde{Y}_{t}^{x} 1\{\tilde{\tau}>t\}$, which by Lemma 6.1 has the same law as $\xi_{t}^{1}-\xi_{t}^{2}$ under $P_{\left\{z_{1}, z_{2}\right\}}$ when $z_{1}-z_{2}=x$. As in Lemma 6.3 we will write $Y_{t}^{\bar{U}}$ when the initial law of $Y_{t}$ is the law of $\bar{U}$.

Lemma 7.4. For $s \geq 0$,

$$
J_{s}^{N, 2}(\mathbf{1})=\rho^{2}\left|B_{r}\right|^{2} N^{1-\frac{d}{2}} \int_{\mathbb{R}^{d}} X_{0}^{N}(y) E\left[X_{0}^{N}\left(y-\frac{\tilde{Y}_{N s}^{\bar{U}}}{\sqrt{N}}\right) 1\{\tilde{\tau}>N s\}\right] d y .
$$

Proof. By changing variables and orders of integration, and using the difference process $\tilde{\xi}_{s}^{N}=\xi_{s}^{N, 1}-\xi_{s}^{N, 2}$, we have

$$
\begin{aligned}
J_{s}^{N, 2}(\mathbf{1}) & \\
& =\rho^{2} N^{1+d / 2} K^{2} \int_{\mathbb{R}^{d}} \int_{B_{r}^{N}} \int_{B_{r}^{N}} E_{\left\{z_{1}, z_{2}\right\}}^{N}\left[w_{0}^{N}\left(x+\xi_{s}^{N, 1}\right) w_{0}^{N}\left(x+\xi_{s}^{N, 2}\right) 1\left\{\tau^{N}>s\right\}\right] d z_{1} d z_{2} d x \\
& =\rho^{2} N^{1+d / 2} K^{2} \int_{B_{r}^{N}} \int_{B_{r}^{N}} \int_{\mathbb{R}^{d}} w_{0}^{N}(y) E_{\left\{z_{1}, z_{2}\right\}}^{N}\left[w_{0}^{N}\left(y-\xi_{s}^{N, 1}+\xi_{s}^{N, 2}\right) 1\left\{\tau^{N}>s\right\}\right] d y d z_{1} d z_{2} \\
& =\rho^{2} N^{1+d / 2} K^{2} \int_{\mathbb{R}^{d}} w_{0}^{N}(y) \int_{B_{r}^{N}} \int_{B_{r}^{N}} E_{\left\{z_{1}, z_{2}\right\}}^{N}\left(w_{0}^{N}\left(y-\tilde{\xi}_{s}^{N}\right) 1\left\{\tau^{N}>s\right\}\right) d z_{1} d z_{2} d y \\
& =\rho^{2} N^{1-d / 2} K^{2} \int_{\mathbb{R}^{d}} w_{0}^{N}(y) \int_{B_{r}} \int_{B_{r}} E_{\left\{z_{1}, z_{2}\right\}}\left[w_{0}^{N}\left(y-\frac{\tilde{\xi}_{N s}}{\sqrt{N}}\right) 1\{\tilde{\tau}>N s\}\right] d y d z_{1} d z_{2} \\
& =\rho^{2} N^{1-d / 2} K^{2} \int_{\mathbb{R}^{d}} w_{0}^{N}(y) \int_{B_{r}} \int_{B_{r}} E\left[w_{0}^{N}\left(y-\frac{\tilde{Y}_{N s}^{z_{1}-z_{2}}}{\sqrt{N}}\right) 1\{\tilde{\tau}>N s\}\right] d y d z_{1} d z_{2}
\end{aligned}
$$

which is (7.13).

Recall $s_{N}$ from (6.19) and define $\delta_{N}$ so that

$$
s_{N}=(\log N)^{-q}, \quad \delta_{N}=(\log N)^{\delta} s_{N}=(\log N)^{\delta-q},
$$

where $\delta \in\left(0, \frac{1}{2}\right]$ and $q>4$ are fixed constants. 
Lemma 7.5. There is a constant $C_{7.15}>0$ such that

$$
\begin{aligned}
\sup _{s \in\left[s_{N}, 2 s_{N}\right]} J_{s}^{N, 2}(\mathbf{1}) & \leq C_{7.15}\left[\frac{\log \log N}{\log N} X_{0}^{N}(\mathbf{1})\right. \\
& \left.+\frac{N^{\frac{1}{2}-\frac{d}{4}}}{s_{N} \log N} \int_{\mathbb{R}^{d}} \int_{\mathbb{R}^{d}} X_{0}^{N}\left(y_{1}\right) X_{0}^{N}\left(y_{2}\right) 1\left\{\left|y_{1}-y_{2}\right| \leq \sqrt{\delta_{N}}\right\} d y_{1} d y_{2}\right] .
\end{aligned}
$$

Proof. Let $s \in\left[s_{N}, 2 s_{N}\right]$, let $t_{N}=N s / 4$, and define

$$
\begin{aligned}
& \mathcal{E}_{1}=N^{1-\frac{d}{2}} \int_{\mathbb{R}^{d}} X_{0}^{N}(y) E\left[X_{0}^{N}\left(y-\frac{\tilde{Y}_{N s}^{\bar{U}}}{\sqrt{N}}\right) 1\left\{\frac{\left|\tilde{Y}_{N s}^{\bar{U}}\right|}{\sqrt{N}}>\sqrt{\delta_{N}}, \tilde{\tau}>N s\right\}\right] d y \\
& \mathcal{E}_{2}=N^{1-\frac{d}{2}} \int_{\mathbb{R}^{d}} X_{0}^{N}(y) E\left[X_{0}^{N}\left(y-\frac{\tilde{Y}_{N s}^{\bar{U}}}{\sqrt{N}}\right) 1\left\{\left|\tilde{Y}_{t_{N}}^{\bar{U}}\right| \leq 2 r, \frac{\left|\tilde{Y}_{N s}^{\bar{U}}\right|}{\sqrt{N}} \leq \sqrt{\delta_{N}}, \tilde{\tau}>N s\right\}\right] d y \\
& \mathcal{E}_{3}=N^{1-\frac{d}{2}} \int_{\mathbb{R}^{d}} X_{0}^{N}(y) E\left[X_{0}^{N}\left(y-\frac{\tilde{Y}_{N s}^{\bar{U}}}{\sqrt{N}}\right) 1\left\{\left|\tilde{Y}_{t_{N}}^{\bar{U}}\right|>2 r, \frac{\left|\tilde{Y}_{N s}^{\bar{U}}\right|}{\sqrt{N}} \leq \sqrt{\delta_{N}}, \tilde{\tau}>N s\right\}\right] d y
\end{aligned}
$$

To prove (7.15), by Lemma 7.4 it suffices to show that each $\mathcal{E}_{i}$ is uniformly bounded in $s \in\left[s_{N}, 2 s_{N}\right]$ by terms in the right side of (7.15).

Since $I^{-1}(N s) \leq N s \leq 2 N s_{N}$, for $k>2 / \delta$ and $N \geq N_{0}(q, r)$ large enough,

$$
\begin{aligned}
& P\left(\frac{\left|\tilde{Y}_{N s}^{\bar{U}}\right|}{\sqrt{N}}>\sqrt{\delta_{N}}, \tau>N s\right) \leq P\left(\sup _{t \leq 2 N s_{N}}\left|Y_{t}^{\bar{U}}\right| \geq \sqrt{N \delta_{N}}\right) \\
& \quad \leq P\left(\sup _{t \leq 2 N s_{N}}\left|Y_{t}^{0}\right| \geq \sqrt{N \delta_{N}}-2 r\right) \leq C \frac{\left(2 N s_{N}\right)^{k}}{\left(\sqrt{N \delta_{N}}-2 r\right)^{2 k}} \leq C\left(s_{N} / \delta_{N}\right)^{k} \leq C /(\log N)^{2},
\end{aligned}
$$

where we have used (5.5). With this bound and $X_{0}^{N}(\cdot) \leq K$ we obtain from the definition of $\mathcal{E}_{1}$ that

$$
\mathcal{E}_{1} \leq N^{1-\frac{d}{2}} K \frac{C}{(\log N)^{2}} \int_{\mathbb{R}^{d}} X_{0}^{N}(y) d y \leq \frac{C}{\log N} X_{0}^{N}(\mathbf{1}) .
$$

The above bound is then extended to all $N \geq 3$ by increasing $C$.

Using $X_{0}^{N}(\cdot) \leq K$ again, we have

$$
\mathcal{E}_{2} \leq N^{1-\frac{d}{2}} K \int_{\mathbb{R}^{d}} X_{0}^{N}(y) P\left(\left|\tilde{Y}_{N s / 4}^{\bar{U}}\right| \leq 2 r\right) d y=K^{\prime} X_{0}^{N}(\mathbf{1}) P\left(\left|\tilde{Y}_{N s / 4}^{\bar{U}}\right| \leq 2 r\right) .
$$

It follows from Lemma 6.4 , taking $\beta=1 / 3$, that for $N \geq N_{0}(q)$,

$$
\mathcal{E}_{2} \leq C \frac{K^{\prime}}{(N s / 4)^{1 / 3}} X_{0}^{N}(\mathbf{1}) \leq C \frac{\log N}{\left(N s_{N} / 4\right)^{1 / 3}} X_{0}^{N}(\mathbf{1}) \leq C \frac{(\log N)^{1+q / 3}}{N^{1 / 3}} X_{0}^{N}(\mathbf{1}) .
$$

As before, the above bound is then valid for all $N \geq 3$ by increasing $C$.

We split $\mathcal{E}_{3}$ into two parts, letting $G(a, b)=\left\{\left|Y_{I^{-1}(u)}^{\overline{\bar{U}}}\right|>2 r\right.$ for all $\left.u \in[a, b]\right\}$. If we let $G_{N}=G(N s / 2, N s)$ then we can write $\mathcal{E}_{3}=\mathcal{E}_{3}^{\prime}+\mathcal{E}_{3}^{\prime \prime}$, where

$$
\begin{aligned}
& \mathcal{E}_{3}^{\prime}=N^{1-\frac{d}{2}} \int_{\mathbb{R}^{d}} X_{0}^{N}(y) E\left[X_{0}^{N}\left(y-\frac{\tilde{Y}_{N s}^{\bar{U}}}{\sqrt{N}}\right) 1_{G_{N}^{c}} 1\left\{\left|\tilde{Y}_{t_{N}}^{\bar{U}}\right|>2 r, \frac{\left|\tilde{Y}_{N s}^{\bar{U}}\right|}{\sqrt{N}} \leq \sqrt{\delta_{N}}, \tilde{\tau}(\bar{U})>N s\right\}\right] d y \\
& \mathcal{E}_{3}^{\prime \prime}=N^{1-\frac{d}{2}} \int_{\mathbb{R}^{d}} X_{0}^{N}(y) E\left[X_{0}^{N}\left(y-\frac{\tilde{Y}_{N s}^{\bar{U}}}{\sqrt{N}}\right) 1_{G_{N}} 1\left\{\left|\tilde{Y}_{t_{N}}^{\bar{U}}\right|>2 r, \frac{\left|\tilde{Y}_{N s}^{\bar{U}}\right|}{\sqrt{N}} \leq \sqrt{\delta_{N}}, \tilde{\tau}(\bar{U})>N s\right\}\right] d y
\end{aligned}
$$

Applying the Markov property at time $t_{N}=N s / 4$, and letting $P_{\tilde{Y}_{t_{N}}^{\bar{U}}(\omega)}$ denote the law of 
$Y$ with $Y_{0}=\tilde{Y}_{t_{N}}^{\bar{U}}(\omega)$,

$$
\begin{aligned}
\mathcal{E}_{3}^{\prime} \leq & K N^{1-\frac{d}{2}} \int_{\mathbb{R}^{2}} X_{0}^{N}(y) P\left(\left\{\left|\tilde{Y}_{t_{N}}^{\bar{U}}\right|>2 r, \tilde{\tau}(\bar{U})>t_{N}\right\} \cap G_{N}^{c}\right) d y \\
= & K^{\prime} E\left[1\left\{\left|\tilde{Y}_{t_{N}}^{\bar{U}}\right|>2 r, \tilde{\tau}(\bar{U})>t_{N}\right\}\right. \\
& \left.\times P_{\tilde{Y}_{t_{N}}^{\bar{U}}}\left(\left|Y\left(I^{-1}(u)\right)\right| \leq 2 r \text { for some } u \in[N s / 4,3 N s / 4]\right)\right] X_{0}^{N}(\mathbf{1}) \\
\leq & K^{\prime} P\left(\tilde{\tau}(\bar{U})>N s_{N} / 4\right) \\
& \times \sup _{|x|>2 r} P\left(Y^{x}\left(I^{-1}(u)\right) \mid \leq 2 r \text { for some } u \in\left[N s_{N} / 4,3 N s_{N} / 2\right]\right) X_{0}^{N}(\mathbf{1}) \\
\leq & C \frac{\log \log N}{\log N} X_{0}^{N}(\mathbf{1}),
\end{aligned}
$$

where we have used Lemma 6.5, $s_{N}=(\log N)^{-q}$, and the fact that $K^{\prime} P\left(\tau(\bar{U})>N s_{N} / 4\right)$ is bounded in $N$ for $d=2$ by Proposition 1.1, and at most one if $d>2$.

Set $t_{N}^{\prime}=3 N s / 4$ and $t_{N}^{\prime \prime}=t_{N}^{\prime}-t_{N}=N s / 2$. By the Markov property of $\tilde{Y}^{\bar{U}}$ at time $t_{N}=N s / 4$,

$$
\begin{aligned}
\mathcal{E}_{3}^{\prime \prime}= & N^{1-\frac{d}{2}} \int_{\mathbb{R}^{d}} X_{0}^{N}(y) E\left[1\left\{\left|\tilde{Y}_{t_{N}}^{\bar{U}}\right|>2 r, \tilde{\tau}(\bar{U})>t_{N}\right\}\right. \\
& \times E_{\tilde{Y}_{t_{N}}^{\bar{U}}}\left(X_{0}^{N}\left(y-\frac{\tilde{Y}_{t_{N}^{\prime}}}{\sqrt{N}}\right) 1\left\{\frac{\left|\tilde{Y}_{t_{N}^{\prime}}\right|}{\sqrt{N}} \leq \sqrt{\delta_{N}}\right\} 1\left\{G\left(t_{N}, t_{N}^{\prime}\right) \cap\left\{\tilde{\tau}>t_{N}^{\prime}\right\}\right)\right] d y .
\end{aligned}
$$

On the event $G\left(t_{N}, t_{N}^{\prime}\right) \cap\left\{\tilde{\tau}>t_{N}^{\prime}\right\}$, for all $u \in\left[t_{N}, t_{N}^{\prime}\right]$,

$$
I^{-1}(u)=I^{-1}\left(t_{N}\right)+\int_{t_{N}}^{u}\left[1-\frac{\psi_{r}\left(\tilde{Y}_{s}\right)}{\rho\left|B_{r}\right|}\right] d s=I^{-1}\left(t_{N}\right)+u-t_{N} .
$$

Using the above and Lemma 6.1, we see that on the above event and for $u$ as above, under $P_{\tilde{Y}_{t_{N}}^{\bar{U}}}$,

$$
\tilde{Y}(u)-\tilde{Y}\left(t_{N}\right)=Y\left(I^{-1}\left(t_{N}\right)+\left(u-t_{N}\right)\right)-Y\left(I^{-1}\left(t_{N}\right)\right):=\hat{Y}^{0}\left(u-t_{N}\right) .
$$

Set $\tilde{\mathcal{F}}_{s}=\mathcal{F}_{I^{-1}(s)}^{Y}$, where we recall that $\mathcal{F}_{t}^{Y}$ is the right-continuous filtration generated by the random walk $Y$. Then $I^{-1}(s)$ is an $\left(\mathcal{F}_{t}^{Y}\right)$-stopping time. By the strong Markov property of $Y, \hat{Y}^{0}$ is a copy of $Y$ starting at 0 and is independent of $\tilde{\mathcal{F}}_{t_{N}}$. Since $\tilde{Y}_{t_{N}}$ is $\tilde{\mathcal{F}}_{t_{N}}$-measurable, we may conclude from (7.17) and (7.16) that

$$
\begin{aligned}
\mathcal{E}_{3}^{\prime \prime} \leq N^{1-\frac{d}{2}} \int_{\mathbb{R}^{2}} X_{0}^{N}(y) E\left[1\left\{\left|\tilde{Y}_{t_{N}}^{\bar{U}}\right|>2 r, \tilde{\tau}(\bar{U})>t_{N}\right\}\right. \\
\left.\quad \times E_{\tilde{Y}_{t_{N}}^{\bar{U}}}\left(X_{0}^{N}\left(y-\left(\frac{\tilde{Y}_{t_{N}}+\hat{Y}_{t_{N}^{\prime \prime}}^{0}}{\sqrt{t_{N}^{\prime \prime}}}\right) \sqrt{\frac{t_{N}^{\prime \prime}}{N}}\right) 1\left\{\left|\frac{\tilde{Y}_{t_{N}}+\hat{Y}_{t_{N}^{\prime \prime}}^{0}}{\sqrt{t_{N}^{\prime \prime}}}\right| \sqrt{\frac{t_{N}^{\prime \prime}}{N}} \leq \sqrt{\delta_{N}}\right\}\right)\right] d y \\
\left.\leq N^{1-\frac{d}{2}} \int_{\mathbb{R}^{2}} X_{0}^{N}(y) E\left[1\left\{\tilde{\tau}(\bar{U})>t_{N}\right),\left|\tilde{Y}_{t_{N}}^{\bar{U}}\right|>2 r\right\}\right] \\
\quad \times \sup _{x} E\left[X_{0}^{N}\left(y-\frac{\hat{Y}_{t_{N}^{\prime \prime}}^{x}}{\sqrt{t_{N}^{\prime \prime}}} \sqrt{\frac{t_{N}^{\prime \prime}}{N}}\right) 1\left\{\left|\frac{\hat{Y}_{t_{N}^{\prime \prime}}^{x}}{\sqrt{t_{N}^{\prime \prime}}}\right| \sqrt{\frac{t_{N}^{\prime \prime}}{N}} \leq \sqrt{\delta_{N}}\right\}\right] d y
\end{aligned}
$$

where $\hat{Y}^{x}=x+\hat{Y}^{0}$. Apply (5.3) to $f(z)=X_{0}^{N}\left(y-z \sqrt{\frac{t_{N}^{\prime \prime}}{N}}\right) 1\left\{|z| \frac{t_{N}^{\prime \prime}}{N} \leq \sqrt{\delta_{N}}\right\}$, to see that, 
uniformly in $x$,

$$
\begin{aligned}
E\left[X_{0}^{N}\right. & \left.\left.\left(y-\frac{\hat{Y}_{t_{N}^{\prime \prime}}^{x}}{\sqrt{t_{N}^{\prime \prime}}}\right) \sqrt{\frac{t_{N}^{\prime \prime}}{N}}\right) 1\left\{\left|\frac{\hat{Y}_{t_{N}^{\prime \prime}}^{x}}{\sqrt{t_{N}^{\prime \prime}}}\right| \sqrt{\frac{t_{N}^{\prime \prime}}{N}} \leq \sqrt{\delta_{N}}\right\}\right] \\
& \leq e^{-2 \rho\left|B_{r}\right| t_{N}^{\prime \prime}} K+C \int_{\mathbb{R}^{d}} X_{0}^{N}\left(y-z \sqrt{\frac{t_{N}^{\prime \prime}}{N}}\right) 1\left\{|z| \sqrt{\frac{t_{N}^{\prime \prime}}{N}} \leq \sqrt{\delta_{N}}\right\} d z \\
& \leq e^{-\rho\left|B_{r}\right| N s_{N}} K+C\left(\frac{N}{t_{N}^{\prime \prime}}\right)^{d / 2} \int_{\mathbb{R}^{d}} X_{0}^{N}\left(y-x^{\prime}\right) 1\left\{\left|x^{\prime}\right| \leq \sqrt{\delta_{N}}\right\} d x^{\prime} \\
& \leq \frac{C}{\log N}+\frac{C}{s_{N}^{d / 2}} \int_{\mathbb{R}^{d}} X_{0}^{N}\left(y-x^{\prime}\right) 1\left\{\left|x^{\prime}\right| \leq \sqrt{\delta_{N}}\right\} d x^{\prime} .
\end{aligned}
$$

Use this and the fact that $P\left(\tilde{\tau}(\bar{U})>t_{N}\right) \leq \frac{C}{K^{\prime}}$ (from Proposition 1.1 if $\left.d=2\right)$ in (7.18), to see that

$$
\begin{aligned}
\mathcal{E}_{3}^{\prime \prime} \leq & C\left(s_{N} N\right)^{1-\frac{d}{2}} P\left(\tilde{\tau}(\bar{U})>t_{N}\right)\left[\frac{X_{0}^{N}(\mathbf{1})}{\log N}\right. \\
& \left.+\frac{1}{s_{N}} \int_{\mathbb{R}^{d}} \int_{\mathbb{R}^{d}} X_{0}^{N}(y) X_{0}^{N}\left(y-x^{\prime}\right) 1\left\{\left|x^{\prime}\right| \leq \sqrt{\delta_{N}}\right\} d x^{\prime} d y\right] \\
\leq & C\left[\frac{X_{0}^{N}(\mathbf{1})}{(\log N)}+\frac{\left(s_{N} N\right)^{1-\frac{d}{2}}}{s_{N} K^{\prime}} \int_{\mathbb{R}^{d}} \int_{\mathbb{R}^{d}} X_{0}^{N}\left(y_{1}\right) X_{0}^{N}\left(y_{2}\right) 1\left\{\left|y_{1}-y_{2}\right| \leq \sqrt{\delta_{N}} d y_{1} d y_{2}\right]\right. \\
\leq & C\left[\frac{X_{0}^{N}(\mathbf{1})}{(\log N)}+\frac{N^{\frac{1}{2}-\frac{d}{4}}}{s_{N} \log N} \int_{\mathbb{R}^{d}} \int_{\mathbb{R}^{d}} X_{0}^{N}\left(y_{1}\right) X_{0}^{N}\left(y_{2}\right) 1\left\{\left|y_{1}-y_{2}\right| \leq \sqrt{\delta_{N}} d y_{1} d y_{2}\right],\right.
\end{aligned}
$$

where the last line is very crude if $d \geq 3$, and is an equality if $d=2$. Combining the bounds for $\mathcal{E}_{1}, \mathcal{E}_{2}, \mathcal{E}_{3}^{\prime}, \mathcal{E}_{3}^{\prime \prime}$, we establish (7.15).

Corollary 7.3, Lemma 7.5, and $q>4$ imply the following:

Lemma 7.6. There is a constant $C_{7.19}=C_{7.19}(\phi)$ such that

$$
\begin{aligned}
\left.\sup _{s \in\left[s_{N}, 2 s_{N}\right]}\left|E\left(\bar{m}_{s}^{N}(\phi)\right)-\rho^{2}\right| B_{r}\right|^{2} \bar{\gamma}^{N}(s) X_{0}^{N}\left(\phi^{2}\right) \mid \leq C_{7.19}\left[\frac{\log \log N}{\log N} X_{0}^{N}(\mathbf{1})\right. \\
\left.\quad+\frac{N^{\frac{1}{2}-\frac{d}{4}}}{s_{N} \log N} \int_{\mathbb{R}^{d}} \int_{\mathbb{R}^{d}} X_{0}^{N}\left(y_{1}\right) X_{0}^{N}\left(y_{2}\right) 1\left\{\left|y_{1}-y_{2}\right| \leq \sqrt{\delta_{N}}\right\} d y_{1} d y_{2}\right] .
\end{aligned}
$$

Remark 7.7. To identify the square function of $M^{N}(\phi)$ we will need to use the above and the Markov property to bound

$$
\left.\left|E\left(\bar{m}_{s}^{N}(\phi) \mid \mathcal{F}_{s-s_{N}}^{N}\right)-\rho^{2}\right| B_{r}\right|^{2} \bar{\gamma}^{N}\left(s_{N}\right) X_{s-s_{N}}^{N}\left(\phi^{2}\right) \mid .
$$

This means we will need to bound the expected value of the last term in (7.19) with $X_{s-s_{N}}^{N}$ replacing $X_{0}^{N}$. For $d \geq 3$ we only need to bound the resulting double integral on the right-hand side of (7.19) by $X_{s-s_{N}}^{N}(\mathbf{1})^{2}$, but for $d=2$ we require the following additional result.

Lemma 7.8. Assume $d=2$. For $T>0$ there exists $C_{7.20}(T)>0$ such that for $\delta_{N} \leq s \leq T$,

$$
\begin{aligned}
E\left[\int_{\mathbb{R}^{2}} \int_{\mathbb{R}^{2}} 1\left\{\left|y_{1}-y_{2}\right| \leq \sqrt{\delta_{N}}\right\} X_{s}^{N}\left(y_{1}\right) X_{s}^{N}\left(y_{2}\right) d y_{1} d y_{2}\right] \\
\leq C_{7.20}(T)\left(\frac{\delta_{N}}{s} X_{0}^{N}(\mathbf{1})^{2}+\delta_{N} \log \left(1 / \delta_{N}\right) X_{0}^{N}(\mathbf{1})\right) .
\end{aligned}
$$




\section{Convergence of SLFV to SBM}

Proof. By the duality equation (3.3) and then a change of variables,

$$
\begin{aligned}
E\left[\int_{\mathbb{R}^{2}}\right. & \left.\int_{\mathbb{R}^{2}} 1\left\{\left|y_{1}-y_{2}\right| \leq \sqrt{\delta_{N}}\right\} X_{s}^{N}\left(y_{1}\right) X_{s}^{N}\left(y_{2}\right) d y_{1} d y_{2}\right] \\
= & (\log N) \int_{\mathbb{R}^{2}} \int_{\mathbb{R}^{2}} 1\left\{\left|y_{1}-y_{2}\right| \leq \sqrt{\delta_{N}}\right\} E_{\left\{y_{1}, y_{2}\right\}}^{N}\left[X_{0}^{N}\left(\xi_{s}^{N, 1}\right) 1\left\{\tau^{N} \leq s\right\}\right] d y_{1} d y_{2} \\
& +\int_{\mathbb{R}^{2}} \int_{\mathbb{R}^{2}} 1\left\{\left|y_{1}-y_{2}\right| \leq \sqrt{\delta_{N}}\right\} E_{\left\{y_{1}, y_{2}\right\}}^{N}\left[X_{0}^{N}\left(\xi_{s}^{N, 1}\right) X_{0}^{N}\left(\xi_{s}^{N, 2}\right) 1\left\{\tau^{N}>s\right\}\right] d y_{1} d y_{2} \\
= & \mathcal{E}_{1}+\mathcal{E}_{2} .
\end{aligned}
$$

Here

$$
\begin{aligned}
\mathcal{E}_{1} & =(\log N) \int_{\mathbb{R}^{2}} \int_{\mathbb{R}^{2}} 1\left\{\left|y_{1}-y_{2}\right| \leq \sqrt{\delta_{N}}\right\} E_{\left\{y_{1}, y_{2}\right\}}^{N}\left[X_{0}^{N}\left(\xi_{s}^{N, 1}\right) 1\left\{\tau^{N} \leq s\right\}\right] d y_{1} d y_{2} \\
& =(\log N) \int_{\mathbb{R}^{2}} \int_{\mathbb{R}^{2}} 1\left\{\left|y_{1}-y_{2}\right| \leq \sqrt{\delta_{N}}\right\} E_{\left\{y_{1}-y_{2}, 0\right\}}^{N}\left[X_{0}^{N}\left(y_{2}+\xi_{s}^{N, 1}\right) 1\left\{\tau^{N} \leq s\right\}\right] d y_{2} d y_{1} \\
& =(\log N) \int_{\mathbb{R}^{2}} \int_{\mathbb{R}^{2}} 1\left\{|y| \leq \sqrt{\delta_{N}}\right\} E_{\{y, 0\}}^{N}\left[X_{0}^{N}\left(y_{2}+\xi_{s}^{N, 1}\right) 1\left\{\tau^{N} \leq s\right\}\right] d y_{2} d y,
\end{aligned}
$$

and

$$
\left.\mathcal{E}_{2}=\int_{\mathbb{R}^{2}} \int_{\mathbb{R}^{2}} 1\left\{|y| \leq \sqrt{\delta_{N}}\right\} E_{\{y, 0\}}^{N}\left[X_{0}^{N}\left(y_{2}+\xi_{s}^{N, 1}\right) X_{0}^{N}\left(y_{2}+\xi_{s}^{N, 2}\right) 1\left\{\tau^{N}>s\right)\right\}\right] d y_{2} d y .
$$

First, integrating $y_{2}$ out in $\mathcal{E}_{1}$ yields

$$
\begin{aligned}
\mathcal{E}_{1} & \leq(\log N) X_{0}^{N}(\mathbf{1}) \int_{\mathbb{R}^{2}} 1\left\{|y| \leq \sqrt{\delta_{N}}\right\} P_{\{y, 0\}}^{N}\left(\tau^{N} \leq s\right) d y \\
& =(\log N) X_{0}^{N}(\mathbf{1}) \int_{\mathbb{R}^{2}} 1\left\{|y| \leq \sqrt{\delta_{N}}\right\} P_{\{y \sqrt{N}, 0\}}(\tau \leq N s) d y .
\end{aligned}
$$

By Lemma 6.1, switching to $\tilde{\xi}^{y \sqrt{N}}$, and using $I^{-1}(u) \leq u$,

$$
\begin{aligned}
P_{\{y \sqrt{N}, 0\}}(\tau \leq N s) & =P\left(\tilde{\xi}_{N s}^{y \sqrt{N}}=0\right) \leq P\left(\inf _{u \leq N s}\left|\tilde{Y}_{u}^{y \sqrt{N}}\right| \leq 3 r\right) \\
& \leq P\left(\inf _{u \leq N s}\left|Y_{u}^{y \sqrt{N}}\right| \leq 3 r\right)=P_{y \sqrt{N}}\left(t_{3 r} \leq N s\right) .
\end{aligned}
$$

By Lemma 5.5, which is applicable because $T \geq s \geq \delta_{N}$ and we consider only $|y| \leq \sqrt{\delta_{N}}$, the last probability above is bounded by $C \frac{\log 1 /|y|}{\log N}(C=C(T))$. It follows that

$$
\begin{aligned}
\mathcal{E}_{1} & \leq C X_{0}^{N}(\mathbf{1}) \int_{\mathbb{R}^{2}} 1\left\{|y| \leq \sqrt{\delta_{N}}\right\} \log (1 /|y|) d y \\
& =C X_{0}^{N}(\mathbf{1}) \int_{0}^{\sqrt{\delta_{N}}} u \log (1 / u) d u \\
& \leq C X_{0}^{N}(\mathbf{1}) \delta_{N} \log \left(1 / \delta_{N}\right) .
\end{aligned}
$$

By definition,

$$
\begin{aligned}
\mathcal{E}_{2} & \left.=\int_{\mathbb{R}^{2}} \int_{\mathbb{R}^{2}} 1\left\{|y| \leq \sqrt{\delta_{N}}\right\} E_{\{y \sqrt{N}, 0\}}\left[X_{0}^{N}\left(y_{2}+\frac{\xi_{s N}^{1}}{\sqrt{N}}\right) X_{0}^{N}\left(y_{2}+\frac{\xi_{s N}^{2}}{\sqrt{N}}\right) 1\{\tau>N s)\right\}\right] d y_{2} d y \\
& =\mathcal{E}_{2}^{\prime}+\mathcal{E}_{2}^{\prime \prime},
\end{aligned}
$$


where, with $G_{N}=\left\{\left|\xi_{u}^{1}-\xi_{u}^{2}\right| \geq 3 r\right.$ for all $\left.u \leq N s\right\}$,

$$
\begin{aligned}
\mathcal{E}_{2}^{\prime}= & \int_{\mathbb{R}^{2}} \int_{\mathbb{R}^{2}} 1\left\{|y| \leq \sqrt{\delta_{N}}\right\} E_{\{y \sqrt{N}, 0\}}\left[X_{0}^{N}\left(y_{2}+\frac{\xi_{s N}^{1}}{\sqrt{N}}\right) X_{0}^{N}\left(y_{2}+\frac{\xi_{s N}^{2}}{\sqrt{N}}\right)\right. \\
& \left.\left.\times 1_{G_{N}} 1\{\tau>N s)\right\}\right] d y_{2} d y, \\
\mathcal{E}_{2}^{\prime \prime}= & \int_{\mathbb{R}^{2}} \int_{\mathbb{R}^{2}} 1\left\{|y| \leq \sqrt{\delta_{N}}\right\} E_{\{y \sqrt{N}, 0\}}\left[X_{0}^{N}\left(y_{2}+\frac{\xi_{s N}^{1}}{\sqrt{N}}\right) X_{0}^{N}\left(y_{2}+\frac{\xi_{s N}^{2}}{\sqrt{N}}\right)\right. \\
& \left.\left.\times 1_{G_{N}^{c}} 1\{\tau>N s)\right\}\right] d y_{2} d y .
\end{aligned}
$$

We use the representation of $\xi=\left(\xi^{1}, \xi^{2}\right)$ in Lemma 6.6 and the fact that on $G_{N} \cap\{\tau>N s\}, \xi_{s N}=W_{s N}$. Then, dropping the indicator of $G_{N} \cap\{\tau>N s\}$, we see that

$$
\begin{aligned}
\mathcal{E}_{2}^{\prime} & \leq \int_{\mathbb{R}^{2}} \int_{\mathbb{R}^{2}} 1\left\{|y| \leq \sqrt{\delta_{N}}\right\} E\left[X_{0}^{N}\left(y_{2}+\frac{W_{s N}^{y \sqrt{N}}}{\sqrt{N}}\right) X_{0}^{N}\left(y_{2}+\frac{W_{s N}^{0}}{\sqrt{N}}\right)\right] d y_{2} d y \\
& =\int_{\mathbb{R}^{2}} \int_{\mathbb{R}^{2}} 1\left\{|y| \leq \sqrt{\delta_{N}}\right\} E\left[X_{0}^{N}\left(y_{2}+\frac{W_{s N}^{y \sqrt{N}}}{\sqrt{N}}\right)\right] E\left[X_{0}^{N}\left(y_{2}+\frac{W_{s N}^{0}}{\sqrt{N}}\right)\right] d y_{2} d y,
\end{aligned}
$$

the last equality by independence of the walks $W^{y \sqrt{N}}, W^{0}$. By the density bound (5.3) applied to $f(z)=X_{0}^{N}\left(y_{2}+z \sqrt{s}\right)$, and $s \geq s_{N}$, for all $y_{2}$,

$$
\begin{aligned}
E\left[X_{0}^{N}\left(y_{2}+\frac{W_{N s}^{0}}{\sqrt{N}}\right)\right] & =E\left[f\left(\frac{W_{N s}^{0}}{\sqrt{N s}}\right)\right] \leq e^{-2 \rho\left|B_{r}\right| N s} \log N+C \int_{\mathbb{R}^{2}} X_{0}^{N}\left(y_{2}-z \sqrt{s}\right) d z \\
& \leq \frac{C}{\log N}+\frac{C}{s} \int_{\mathbb{R}^{2}} X_{0}^{N}\left(y_{2}-z^{\prime}\right) d z^{\prime}=\frac{C}{\log N}+\frac{C}{s} X_{0}^{N}(\mathbf{1}) .
\end{aligned}
$$

With this bound, integrating out $y_{2}$ first and then $y$, it follows that

$$
\begin{aligned}
\mathcal{E}_{2}^{\prime} & \leq C\left(\frac{1}{\log N}+\frac{1}{s} X_{0}^{N}(\mathbf{1})\right) \int_{\mathbb{R}^{2}} \int_{\mathbb{R}^{2}} 1\left\{|y| \leq \sqrt{\delta_{N}}\right\} E\left[X_{0}^{N}\left(y_{2}+\frac{W_{N s}^{y \sqrt{N}}}{\sqrt{N}}\right)\right] d y_{2} d y \\
& =C\left(\frac{1}{\log N}+\frac{1}{s} X_{0}^{N}(\mathbf{1})\right) X_{0}^{N}(\mathbf{1})\left|B_{\sqrt{\delta_{N}}}\right| \leq C\left[\frac{1}{\log N}+\frac{1}{s} X_{0}^{N}(\mathbf{1})\right] X_{0}^{N}(\mathbf{1}) \delta_{N} .
\end{aligned}
$$

Using $X_{0}^{N}(\cdot) \leq K^{\prime}=\log N$ and integrating out $y_{2}$ gives

$$
\left.\mathcal{E}_{2}^{\prime \prime} \leq(\log N) X_{0}^{N}(\mathbf{1}) \int_{\mathbb{R}^{2}} 1\left\{|y| \leq \sqrt{\delta_{N}}\right\} P_{\{y \sqrt{N, 0\}}}\left(G_{N}^{c} \cap\{\tau>N s)\right\}\right) d y .
$$

By the representation of $\xi=\left(\xi^{1}, \xi^{2}\right)$ given in Lemma 6.6, for any $y$,

$$
\left.\left.P_{\{y \sqrt{N}, 0\}}\left(G_{N}^{c} \cap\{\tau>N s)\right\}\right) \leq P\left(\mid W_{u}^{y \sqrt{N}}-W_{u}^{0}\right) \mid \leq 3 r \text { for some } u \leq N s\right) .
$$

Using Lemma 5.5 again, for $\delta_{N} \leq s \leq T$, we have

$$
\begin{aligned}
\mathcal{E}_{2}^{\prime \prime} & \leq(\log N) X_{0}^{N}(\mathbf{1}) \int_{\mathbb{R}^{2}} 1\left\{|y| \leq \sqrt{\delta_{N}}\right\} P\left(\left|Y_{u}^{y \sqrt{N}}\right| \leq 3 r \text { for some } u \leq N s\right) d y \\
& \leq C(T) X_{0}^{N}(\mathbf{1}) \int_{\mathbb{R}^{2}} 1\left\{|y| \leq \sqrt{\delta_{N}}\right\} \log (1 /|y|) d y \\
& \leq C(T) X_{0}^{N}(\mathbf{1}) \delta_{N} \log \left(1 / \delta_{N}\right) .
\end{aligned}
$$

Combining the bounds on $\mathcal{E}_{1}, \mathcal{E}_{2}^{\prime}, \mathcal{E}_{3}^{\prime \prime}$ gives (7.20). 


\section{Convergence of SLFV to SBM}

We are almost ready for the proof of Proposition 4.1. The proof is lengthy, so we separate out one of its key steps in the following lemma. For $s \geq s_{N}$ define

$$
[s]_{N}=(j-1) s_{N} \text { if } s \in\left[j s_{N},(j+1) s_{N}\right), \quad j \geq 1 .
$$

For $T>0$ define $j_{T}=j_{T}(N) \in \mathbb{N}$ by

$$
\left(j_{T}-1\right) s_{N} \leq T<j_{T} s_{N}
$$

Lemma 7.9. Assume $\phi \in C_{0}^{3}\left(\mathbb{R}^{d}\right)$ and $T>0$. There exists $\varepsilon_{7.9}^{N}=\varepsilon_{7.9}^{N}(\phi, T) \rightarrow 0$ as $N \rightarrow \infty$ such that

$$
E\left[\sup _{s_{N} \leq t \leq T}\left|\int_{s_{N}}^{t}\left(\bar{m}_{s}^{N}(\phi)-E\left(\bar{m}_{s}^{N}(\phi) \mid \mathcal{F}_{[s]_{N}}^{N}\right)\right) d s\right|^{2}\right] \leq \varepsilon_{7.9}^{N}\left(X_{0}^{N}(\mathbf{1})^{2}+1\right)
$$

Proof. Define

$$
\Delta_{j}^{N}=\int_{(j-1) s_{N}}^{j s_{N}}\left(\bar{m}_{s}^{N}(\phi)-E\left(\bar{m}_{s}^{N}(\phi) \mid \mathcal{F}_{[s]_{N}}^{N}\right)\right) d s, \quad j \geq 2 .
$$

Then

$$
\int_{s_{N}}^{j s_{N}}\left(\bar{m}_{s}^{N}(\phi)-E\left(\bar{m}_{s}^{N}(\phi) \mid \mathcal{F}_{[s]_{N}}^{N}\right) d s=\sum_{i=2}^{j} \Delta_{i}^{N}=Q_{j}^{N, o}+Q_{j}^{N, e}\right.
$$

where

$$
Q_{j}^{N, o}=\sum_{i=2}^{j} \Delta_{i}^{N} 1\{i \text { is odd }\} \text { and } Q_{j}^{N, e}=\sum_{i=2}^{j} \Delta_{i}^{N} 1\{i \text { is even }\} .
$$

Noting that $\mathcal{F}_{[s]_{N}}^{N}=\mathcal{F}_{(2 i-1) s_{N}}^{N}$ for all $s \in\left[2 i s_{N},(2 i+1) s_{N}\right)$, we see that for $i \geq 1$,

$$
\begin{aligned}
E\left(\Delta_{2 i+1}^{N} \mid \mathcal{F}_{(2 i-1) s_{N}}^{N}\right) & =\int_{2 i s_{N}}^{(2 i+1) s_{N}} E\left[\left(\bar{m}_{s}^{N}(\phi)-E\left(\bar{m}_{s}^{N}(\phi) \mid \mathcal{F}_{[s]_{N}}^{N}\right)\right) \mid \mathcal{F}_{(2 i-1) s_{N}}^{N}\right] d s \\
& =\int_{2 i s_{N}}^{(2 i+1) s_{N}}\left(E\left(\bar{m}_{s}^{N}(\phi) \mid \mathcal{F}_{(2 i-1) s_{N}}^{N}\right)-E\left(\bar{m}_{s}^{N}(\phi) \mid \mathcal{F}_{(2 i-1) s_{N}}^{N}\right)\right) d s \\
& =0,
\end{aligned}
$$

which shows that $\left(Q_{2 j-1}^{N, o}, j \geq 2\right)$ is an $\left(\mathcal{F}_{(2 j-1) s_{N}}^{N}\right)$ martingale. Similarly, $\left(Q_{2 j}^{N, e}, j \geq 1\right)$ is an $\left(\mathcal{F}_{2 j s_{N}}^{N}\right)$ martingale. Using Doob's $L^{2}$ inequality, we have

$$
\begin{aligned}
E\left[\sup _{2 \leq j \leq j_{T}} \mid\right. & \left.\left|\int_{s_{N}}^{j s_{N}}\left(\bar{m}_{s}^{N}(\phi)-E\left(\bar{m}_{s}^{N}(\phi) \mid \mathcal{F}_{[s]_{N}}^{N}\right)\right) d s\right|^{2}\right] \\
& =E\left[\sup _{2 \leq j \leq j_{T}}\left|Q_{j}^{N, o} 1_{\{j \text { odd }\}}+Q_{j}^{N, e} 1_{\{j \text { even }\}}\right|^{2}\right] \\
& \leq 2 E\left[\sup _{2 \leq j \leq j_{T}, j \text { odd }}\left(Q_{j}^{N, o}\right)^{2}+\sup _{2 \leq j \leq J_{T}, j \text { even }}\left(Q_{j}^{N, e}\right)^{2}\right] \leq C E\left[\left(Q_{j_{T}}^{N, o}\right)^{2}+\left(Q_{j_{T}}^{N, e}\right)^{2}\right] .
\end{aligned}
$$

Now if $j_{T}=2 k-1$, then by (7.22),

$$
\begin{aligned}
E\left[\left(Q_{j_{T}}^{N, o}\right)^{2}\right]= & \sum_{i=2}^{k} E\left[\left(\Delta_{2 i-1}^{N}\right)^{2}\right] \leq \sum_{i=2}^{j_{T}} E\left[\left(\Delta_{i}^{N}\right)^{2}\right] \\
\leq & 2 \sum_{i=2}^{j_{T}} E\left[\left(\int_{(i-1) s_{N}}^{i s_{N}} \bar{m}_{s}^{N}(\phi) d s\right)^{2}\right] \\
& +2 \sum_{i=2}^{j_{T}} E\left[\left(\int_{(i-1) s_{N}}^{i s_{N}} E\left(\bar{m}_{s}^{N}(\phi) \mid \mathcal{F}_{(i-1) s_{N}}^{N}\right) d s\right)^{2}\right],
\end{aligned}
$$




\section{Convergence of SLFV to SBM}

which also holds if $j_{T}$ is even. Define $\bar{X}_{T}^{N}(\mathbf{1})=\sup _{t \leq T} X_{t}^{N}(\mathbf{1})$. By Lemma 2.4(a), (3.5) and (3.10) we have

$$
\begin{aligned}
\sum_{i=2}^{j_{T}} E\left[\left(\int_{(i-1) s_{N}}^{i s_{N}} \bar{m}_{s}^{N}(\phi) d s\right)^{2}\right] & \leq \sum_{i=2}^{j_{T}} E\left[\left(\int_{(i-1) s_{N}}^{i s_{N}} C_{\phi}(\log N) X_{s}^{N}(\mathbf{1}) d s\right)^{2}\right] \\
& \leq C_{\phi}(\log N)^{2} \sum_{i=2}^{j_{T}} s_{N}^{2} E\left[\bar{X}_{T+1}^{N}(\mathbf{1})^{2}\right] \\
& \leq C(\phi, T)\left(X_{0}^{N}(\mathbf{1})^{2}+1\right)\left(j_{T} s_{N}\right) s_{N}(\log N)^{2} \\
& \leq C(\phi, T)\left(X_{0}^{N}(\mathbf{1})^{2}+1\right)(\log N)^{2-q} .
\end{aligned}
$$

This and (7.24) imply $E\left[\left(Q_{j_{T}}^{N, o}\right)^{2}\right] \leq C(\phi, T)\left(X_{0}^{N}(1)^{2}+1\right)(\log N)^{2-q}$, and it is clear this bound also holds for $E\left[\left(Q_{j_{T}}^{N, e}\right)^{2}\right]$. Plugging into (7.23) we obtain

$$
E\left[\sup _{2 \leq j \leq j_{T}}\left|\int_{s_{N}}^{j s_{N}}\left(\bar{m}_{s}^{N}(\phi)-E\left(\bar{m}_{s}^{N}(\phi) \mid \mathcal{F}_{[s]_{N}}^{N}\right)\right) d s\right|^{2}\right] \leq C(\phi, T)\left(X_{0}^{N}(1)^{2}+1\right)(\log N)^{2-q} .
$$

Now if $(i-1) s_{N} \leq t \leq i s_{N}$, then

$$
\begin{aligned}
& \left(\int_{s_{N}}^{t}\left(\bar{m}_{s}^{N}(\phi)-E\left(\bar{m}_{s}^{N}(\phi) \mid \mathcal{F}_{[s]_{N}}^{N}\right)\right) d s\right)^{2} \\
& \leq\left(\left|\int_{s_{N}}^{(i-1) s_{N}}\left(\bar{m}_{s}^{N}(\phi)-E\left(\bar{m}_{s}^{N}(\phi) \mid \mathcal{F}_{[s]_{N}}^{N}\right)\right) d s\right|\right. \\
& \left.\quad+\int_{(i-1) s_{N}}^{i s_{N}}\left(\bar{m}_{s}^{N}(\phi)+E\left(\bar{m}_{s}^{N}(\phi) \mid \mathcal{F}_{[s]_{N}}^{N}\right)\right) d s\right)^{2} \\
& \quad \leq 2\left(\int_{s_{N}}^{(i-1) s_{N}}\left(\bar{m}_{s}^{N}(\phi)-E\left(\bar{m}_{s}^{N}(\phi) \mid \mathcal{F}_{[s]_{N}}^{N}\right)\right) d s\right)^{2}+2\left(2 C_{\phi}(\log N) s_{N} \bar{X}_{T+1}^{N}(\mathbf{1})\right)^{2} \\
& \leq 2 \sup _{2 \leq j \leq j_{T}}\left(\int_{s_{N}}^{j s_{N}}\left(\bar{m}_{s}^{N}(\phi)-E\left(\bar{m}_{s}^{N}(\phi) \mid \mathcal{F}_{[s]_{N}}^{N}\right)\right) d s\right)^{2}+C_{\phi}(\log N)^{2-2 q} \bar{X}_{T+1}^{N}(\mathbf{1})^{2}
\end{aligned}
$$

where we have used Lemma 2.4(a), (3.5), and the martingale property of $X_{s}^{N}(\mathbf{1})$ (Corollary 3.2). Thus from the above, (7.26), and Corollary 3.2, the left side of (7.21) is bounded above by

$$
\begin{array}{r}
2 E\left[\sup _{2 \leq j \leq j_{T}}\left(\int_{s_{N}}^{j s_{N}}\left(\bar{m}_{s}^{N}(\phi)-E\left(\bar{m}_{s}^{N}(\phi) \mid \mathcal{F}_{[s]_{N}}^{N}\right)\right) d s\right)^{2}\right]+C_{\phi}(\log N)^{2-2 q} E\left[\bar{X}_{T+1}^{N}(\mathbf{1})^{2}\right] \\
\leq C(\phi, T)\left(X_{0}^{N}(1)^{2}+1\right)(\log N)^{2-q}
\end{array}
$$

Define $\tilde{\gamma}^{N}(s)=\bar{\gamma}^{N}\left(s-[s]_{N}\right)=K^{\prime} \gamma_{e}\left(N\left(s-[s]_{N}\right)\right)$. Note that for $s \geq s_{N}, s-[s]_{N} \geq s_{N}$, and so by Proposition 1.1,

$$
\tilde{\gamma}^{N}(s) \rightarrow \gamma_{e} \text { uniformly in } s \geq s_{N} \text { as } N \rightarrow \infty,
$$

and there is a constant $C_{\gamma}>0$ such that

$$
\sup _{N \geq 3, s \geq s_{N}} \tilde{\gamma}^{N}(s) \leq C_{\gamma} .
$$




\section{Convergence of SLFV to SBM}

Proof of Proposition 4.1. It follows from Proposition 2.2, Lemma 2.4, and Corollary 3.2 that

$$
E\left[\sup _{0 \leq t \leq T}\left|\left\langle M^{N}(\phi)\right\rangle_{t}-\int_{0}^{t} \bar{m}_{s}^{N}(\phi) d s\right|\right] \leq C \frac{\log N}{\sqrt{N}} \int_{0}^{T} E\left(X_{s}^{N}(\mathbf{1})\right) d s=C T \frac{\log N}{\sqrt{N}} X_{0}^{N}(\mathbf{1}),
$$

and so to prove (4.1) it suffices to show

$$
\sup _{X_{0}^{N}(\mathbf{1}) \leq A} E\left[\sup _{0 \leq t \leq T}\left|\int_{0}^{t}\left(\bar{m}_{s}^{N}(\phi)-\rho^{2}\left|B_{r}\right|^{2} \gamma_{e} X_{s}^{N}\left(\phi^{2}\right)\right) d s\right|\right] \rightarrow 0 \text { as } N \rightarrow \infty .
$$

Furthermore, by Lemma 2.4(a), Proposition 3.1(a) and Corollary 3.2,

$$
E\left(\bar{m}_{s}^{N}(\phi)+\rho^{2}\left|B_{r}\right|^{2} \gamma_{e} X_{s}^{N}\left(\phi^{2}\right)\right) \leq E\left(C K^{\prime} X_{s}^{N}(\mathbf{1})\right)=C K^{\prime} X_{0}^{N}(\mathbf{1}),
$$

and therefore

$$
E\left[\int_{0}^{3 \delta_{N}}\left(\bar{m}_{s}^{N}(\phi)+\rho^{2}\left|B_{r}\right|^{2} \gamma_{e} X_{s}^{N}\left(\phi^{2}\right)\right) d s\right] \leq C K^{\prime} \delta_{N} X_{0}^{N}(\mathbf{1}) \leq C(\log N)^{1+\delta-q} X_{0}^{N}(\mathbf{1}) .
$$

So by our choice of $\delta, q$ (recall (7.14)), we need only integrate over $\left[3 \delta_{N}, T\right]$ in (7.30).

Let us write

$$
E\left[\sup _{3 \delta_{N} \leq t \leq T}\left|\int_{3 \delta_{N}}^{t}\left(\bar{m}_{t}^{N}(\phi)-\rho^{2}\left|B_{r}\right|^{2} \gamma_{e} X_{s}^{N}\left(\phi^{2}\right)\right) d s\right|\right] \leq \mathcal{E}_{1}+\mathcal{E}_{2}+\mathcal{E}_{3}+\mathcal{E}_{4},
$$

where

$$
\begin{aligned}
& \mathcal{E}_{1}=E\left[\sup _{3 \delta_{N} \leq t \leq T}\left|\int_{3 \delta_{N}}^{t}\left(\bar{m}_{s}^{N}(\phi)-E\left(\bar{m}_{s}^{N}(\phi) \mid \mathcal{F}_{\left.[s]_{N}\right]}\right)\right) d s\right|\right], \\
& \mathcal{E}_{2}=E\left[\left.\int_{3 \delta_{N}}^{T}\left|E\left(\bar{m}_{s}^{N}(\phi) \mid \mathcal{F}_{[s]_{N}}\right)-\rho^{2}\right| B_{r}\right|^{2} \tilde{\gamma}^{N}(s) X_{[s]_{N}}^{N}\left(\phi^{2}\right) \mid d s\right], \\
& \mathcal{E}_{3}=\rho^{2}\left|B_{r}\right|^{2} E\left[\int_{3 \delta_{N}}^{T} \tilde{\gamma}^{N}(s)\left|X_{[s]_{N}}^{N}\left(\phi^{2}\right)-X_{s}^{N}\left(\phi^{2}\right)\right| d s\right], \\
& \mathcal{E}_{4}=\rho^{2}\left|B_{r}\right|^{2} E\left[\int_{3 \delta_{N}}^{T}\left|\tilde{\gamma}^{N}(s)-\gamma_{e}\right| X_{s}^{N}\left(\phi^{2}\right) d s\right] .
\end{aligned}
$$

By Lemma 7.9,

$$
\mathcal{E}_{1}^{2} \leq \varepsilon_{7.9}^{N}\left(X_{0}^{N}(\mathbf{1})^{2}+1\right)
$$

The error term $\mathcal{E}_{4}$ is simple:

$$
\mathcal{E}_{4} \leq C \sup _{s \geq 3 \delta_{N}}\left|\tilde{\gamma}^{N}(s)-\gamma_{e}\right| \rho^{2}\left|B_{r}\right|^{2} \int_{3 \delta_{N}}^{T} E\left(X_{s}^{N}(1)\right) d s \leq C T \sup _{s \geq 3 \delta_{N}}\left|\tilde{\gamma}^{N}(s)-\gamma_{e}\right| X_{0}^{N}(\mathbf{1}) \rightarrow 0
$$

as $N \rightarrow \infty$, by (7.28) and $s \geq 3 \delta_{N} \geq s_{N}$.

We now consider $\mathcal{E}_{3}$. Let $j_{T}$ be as defined before Lemma 7.9, and let $j_{0} \in \mathbb{N}$ be defined by

$$
\left(j_{0}-1\right) s_{N} \leq 3 \delta_{N}<j_{0} s_{N} .
$$

By the martingale decomposition in Proposition 2.2 and then Cauchy-Schwarz,

$$
\begin{aligned}
E\left(\left|X_{s}^{N}\left(\phi^{2}\right)-X_{[s]_{N}}^{N}\left(\phi^{2}\right)\right|\right) & \left.\leq E\left(\mid D_{s}^{N}\left(\phi^{2}\right)\right)-D_{[s]_{N}}^{N}\left(\phi^{2}\right) \mid\right)+E\left(\left|M_{s}^{N}\left(\phi^{2}\right)-M_{[s]_{N}}^{N}\left(\phi^{2}\right)\right|\right) \\
& \leq \int_{[s]_{N}}^{s} E\left(\left|d_{u}^{N}\left(\phi^{2}\right)\right|\right) d u+\left[E\left(\left\langle M^{N}\left(\phi^{2}\right)\right\rangle_{s}-\left\langle M^{N}\left(\phi^{2}\right)\right\rangle_{[s]_{N}}\right)\right]^{1 / 2} .
\end{aligned}
$$




\section{Convergence of SLFV to SBM}

By Lemma 2.3 and Corollary 3.2,

$$
\int_{[s]_{N}}^{s} E\left(\left|d_{u}^{N}\left(\phi^{2}\right)\right|\right) d u \leq C_{\phi} \int_{[s]_{N}}^{s} X_{0}^{N}(\mathbf{1}) d u \leq C_{\phi} 2 s_{N} X_{0}^{N}(\mathbf{1}) .
$$

By Proposition 2.2, Lemma 2.4 and (7.31),

$$
\begin{aligned}
E\left(\left\langle M^{N}\left(\phi^{2}\right)\right\rangle_{s}-\left\langle M^{N}\left(\phi^{2}\right)\right\rangle_{[s]_{N}}\right) & =E\left[E_{X_{[s]_{N}}^{N}}\left(\int_{0}^{s-[s]_{N}} m_{u}^{N}\left(\phi^{2}\right) d u\right)\right] \\
& \leq C_{\phi}(\log N) \int_{0}^{2 s_{N}} E\left(X_{u}^{N}(\mathbf{1})\right) d u \\
& =C_{\phi}(\log N) s_{N} X_{0}^{N}(\mathbf{1}) .
\end{aligned}
$$

Plugging these bounds into (7.35), and using (7.29) and (7.14), we obtain

$$
\mathcal{E}_{3} \leq C T\left((\log N)^{-q} X_{0}^{N}(\mathbf{1})+(\log N)^{\frac{1-q}{2}} X_{0}^{N}(\mathbf{1})^{\frac{1}{2}}\right)
$$

Turning to $\mathcal{E}_{2}$, first use the Markov property and Lemma 7.6, and then Corollary 3.2, to conclude that

$$
\begin{aligned}
\mathcal{E}_{2} \leq E & {\left[\int _ { 3 \delta _ { N } } ^ { T } C \left[\frac{\log \log N}{\log N} X_{[s]_{N}}^{N}(\mathbf{1})\right.\right.} \\
& \left.\left.+\frac{N^{\frac{1}{2}-\frac{d}{4}}}{s_{N} \log N} \int_{\mathbb{R}^{d}} \int_{\mathbb{R}^{d}} 1\left\{\left|y_{1}-y_{2}\right| \leq \sqrt{\delta_{N}}\right\} X_{[s]_{N}}^{N}\left(y_{1}\right) X_{[s]_{N}}^{N}\left(y_{2}\right) d y_{1} d y_{2}\right] d s\right] \\
\leq & C T \frac{\log \log N}{\log N} X_{0}^{N}(\mathbf{1}) \\
& +\frac{C N^{\frac{1}{2}-\frac{d}{4}}}{s_{N} \log N} \int_{3 \delta_{N}}^{T} \int_{\mathbb{R}^{d}} \int_{\mathbb{R}^{d}} 1\left\{\left|y_{1}-y_{2}\right| \leq \sqrt{\delta_{N}}\right\} E\left(X_{[s]_{N}}^{N}\left(y_{1}\right) X_{[s]_{N}}^{N}\left(y_{2}\right)\right) d y_{1} d y_{2} d s .
\end{aligned}
$$

If $d \geq 3$, use Corollary 3.2 to bound the last term by

$$
\frac{C_{T} N^{\frac{1}{2}-\frac{d}{4}}}{s_{N} \log N}\left(X_{0}(\mathbf{1})+X_{0}^{N}(\mathbf{1})^{2}\right) .
$$

If $d=2$, then by Lemma 7.8,

$$
\begin{array}{rl}
\int_{3 \delta_{N}}^{T} \int_{\mathbb{R}^{d}} \int_{\mathbb{R}^{d}} & 1\left\{y_{1}-y_{2} \mid \leq \sqrt{\delta_{N}}\right\} E\left(X_{[s]_{N}}^{N}\left(y_{1}\right) X_{[s]_{N}}^{N}\left(y_{2}\right)\right) d y_{1} d y_{2} d s \\
\leq & C_{T} \int_{3 \delta_{N}}^{T}\left[\frac{\delta_{N}}{[s]_{N}} X_{0}^{N}(\mathbf{1})^{2}+\delta_{N} \log \left(1 / \delta_{N}\right) X_{0}^{N}(\mathbf{1})\right] d s \\
\leq & C_{T} \delta_{N} X_{0}^{N}(\mathbf{1})^{2} \int_{\delta_{N}}^{T} \frac{1}{u} d u+C_{T} T \delta_{N} \log \left(1 / \delta_{N}\right) X_{0}^{N}(\mathbf{1}) \\
& =C_{T} \delta_{N} \log \left(T / \delta_{N}\right)\left(X_{0}^{N}(\mathbf{1})^{2}+X_{0}^{N}(\mathbf{1})\right) .
\end{array}
$$

Now insert the above or (7.38) into (7.37) to arrive at

$$
\begin{aligned}
\mathcal{E}_{2} & \leq C_{T} \frac{\log \log N}{\log N} X_{0}^{N}(\mathbf{1})+C_{T} \frac{\delta_{N} \log \left(1 / \delta_{N}\right)+N^{\frac{1}{2}-\frac{d}{4}}}{s_{N} \log N}\left(X_{0}^{N}(\mathbf{1})^{2}+1\right) \\
& \leq C_{T} \frac{\log \log N}{\log N} X_{0}^{N}(\mathbf{1})+C_{T} \frac{(\log N)^{\delta} \log \log N}{\log N}\left(X_{0}^{N}(\mathbf{1})^{2}+1\right),
\end{aligned}
$$

where the last inequality is rather crude if $d \geq 3$. The required result now follows from (7.32), (7.33), (7.34), (7.36) and (7.39). 


\section{Appendix: Proof of Proposition 1.1}

From the discussion following the statement of Proposition 1.1 we may assume $d=2$ throughout. Recall the definitions and time change construction from Section 6 (especially Lemmas 6.1 and 6.2), using the rate $2 \rho\left|B_{r}\right|$ random walk $Y_{t}$, the difference process $\tilde{\xi}_{t}^{x}$, and absorption time $\tilde{\tau}=\kappa$. By Lemma 6.2, if $x=x_{1}-x_{2}$,

$$
P_{x}(\tilde{\tau}>t)=P_{x}(\kappa>t)=E_{x}\left[\exp \left(-\int_{0}^{I^{-1}(t)} k\left(Y_{s}\right) d s\right)\right]
$$

Thus, to prove (1.16) in Proposition 1.1 we need to show existence and positivity of

$$
\lim _{t \rightarrow \infty}(\log t) E_{x}\left[\exp \left(-\int_{0}^{I^{-1}(t)} k\left(Y_{s}\right) d s\right)\right], \quad x \neq 0 .
$$

As the exact form of the killing rate $k(x)$ will not be important in our arguments, we will replace it with a general radial function $\phi: \mathbb{R}^{2} \rightarrow[0, \infty]$ satisfying

$$
\phi(x)<\infty \text { for } x \neq 0, \phi(x) \text { is } \downarrow \text { in }|x| \text { and } \phi(x)=0 \text { for }|x|>2 r,
$$

where $\downarrow$ in $|x|$ means non-increasing in $|x|$, and similarly for $\uparrow$. We assume throughout that $\phi$ has these properties. Recall the stopping times $t_{A}$ and $T_{A}$ from (5.7).

Proposition 8.1. Let $Y_{0}=x \in \mathbb{R}^{2},|x|>2 r$, or $Y_{0}=\bar{U}$. If there is a constant $c_{\phi}\left(Y_{0}\right)>0$ such that the limit

$$
c_{\phi}\left(Y_{0}\right)=\lim _{A \rightarrow \infty} \log \left(A^{2}\right) E_{Y_{0}}\left[\exp \left(-\int_{0}^{T_{A}} \phi\left(Y_{s}\right) d x\right)\right]
$$

exists, then

$$
\lim _{t \rightarrow \infty}(\log t) E_{Y_{0}}\left[\exp \left(-\int_{0}^{I^{-1}(t)} \phi\left(Y_{s}\right) d s\right)\right]=c_{\phi}\left(Y_{0}\right) .
$$

Proof. Suppose (8.3) holds. We first prove that this implies

$$
\lim _{t \rightarrow \infty}(\log t) E_{Y_{0}}\left[\exp \left(-\int_{0}^{t} \phi\left(Y_{s}\right) d s\right)\right]=c_{\phi}\left(Y_{0}\right) .
$$

If we let $\Gamma_{A}$ be the event $\left\{A^{2} / \log A \leq T_{A} \leq A^{2} \log A\right\}$, then by Lemma 5.4, for $A>$ $\left(2\left|Y_{0}\right|\right) \vee 2, P_{Y_{0}}\left(\Gamma_{A}^{c}\right) \leq C /(\log A)^{2}$. Thus

$$
\begin{aligned}
E_{Y_{0}}\left[\exp \left(-\int_{0}^{T_{A}} \phi\left(Y_{s}\right) d s\right)\right] & \leq E_{Y_{0}}\left[1_{\Gamma_{A}} \exp \left(-\int_{0}^{T_{A}} \phi\left(Y_{s}\right) d s\right)\right]+P_{Y_{0}}\left(\Gamma_{A}^{c}\right) \\
& \leq E_{Y_{0}}\left[\exp \left(-\int_{0}^{A^{2} / \log A} \phi\left(Y_{s}\right) d s\right)\right]+\frac{C}{(\log A)^{2}} .
\end{aligned}
$$

It follows that

$$
\begin{aligned}
& \log \left(\frac{A^{2}}{\log A}\right) E_{Y_{0}}\left[\exp \left(-\int_{0}^{A^{2} / \log A} \phi\left(Y_{s}\right) d s\right)\right] \\
& \quad \geq \log \left(\frac{A^{2}}{\log A}\right)\left(E_{Y_{0}}\left[\exp \left(-\int_{0}^{T_{A}} \phi\left(Y_{s}\right) d s\right)\right]-\frac{C}{(\log A)^{2}}\right) \rightarrow c_{\phi}\left(Y_{0}\right) \text { as } A \rightarrow \infty .
\end{aligned}
$$

This proves

$$
\liminf _{t \rightarrow \infty}(\log t) E_{Y_{0}}\left[\exp \left(-\int_{0}^{t} \phi\left(Y_{s}\right) d x\right)\right] \geq c_{\phi}\left(Y_{0}\right)
$$


Similarly,

$$
\begin{aligned}
E_{Y_{0}}\left[\exp \left(-\int_{0}^{T_{A}} \phi\left(Y_{s}\right) d s\right)\right] & \geq E_{Y_{0}}\left[1_{\Gamma_{A}} \exp \left(-\int_{0}^{T_{A}} \phi\left(Y_{s}\right) d s\right)\right] \\
& \geq E_{Y_{0}}\left[\exp \left(-\int_{0}^{A^{2} \log A} \phi\left(Y_{s}\right) d s\right)\right]-P_{Y_{0}}\left(\Gamma_{A}^{c}\right) .
\end{aligned}
$$

It follows that

$$
\begin{aligned}
\log \left(A^{2} \log A\right) E_{Y_{0}}[\exp & \left.\left(-\int_{0}^{A^{2} \log A} \phi\left(Y_{s}\right) d s\right)\right] \\
\leq & \log \left(A^{2} \log A\right)\left(E_{Y_{0}}\left[\exp \left(-\int_{0}^{T_{A}} \phi\left(Y_{s}\right) d s\right)\right]+\frac{C}{(\log A)^{2}}\right) \\
& \rightarrow c_{\phi}\left(Y_{0}\right) \text { as } A \rightarrow \infty .
\end{aligned}
$$

Along with (8.6), this proves (8.5).

We can now prove (8.4). By (8.1), (8.5) and $I^{-1}(t) \leq t$,

$\liminf _{t \rightarrow \infty}(\log t) E_{x}\left[\exp \left(-\int_{0}^{I^{-1}(t)} \phi\left(Y_{s}\right) d s\right)\right] \geq \lim _{t \rightarrow \infty}(\log t) E_{Y_{0}}\left[\exp \left(-\int_{0}^{t} \phi\left(Y_{s}\right) d s\right)\right]=c_{\phi}\left(Y_{0}\right)$.

On the other hand, by Lemma 6.3, taking $\alpha=1 / 2$,

$$
\begin{aligned}
(\log t) E_{x} & {\left[\exp \left(-\int_{0}^{I^{-1}(t)} \phi\left(Y_{s}\right) d s\right)\right] \leq(\log t) E_{Y_{0}}\left[1\left\{I^{-1}(t) \geq t-t^{1 / 2}\right\}\right.} \\
& \left.\times \exp \left(-\int_{0}^{I^{-1}(t)} \phi\left(Y_{s}\right) d s\right)\right]+P_{Y_{0}}\left(I^{-1}(t)<t-t^{1 / 2}\right) \\
\leq & (\log t) E_{Y_{0}}\left[\exp \left(-\int_{0}^{t-\sqrt{t}} \phi\left(Y_{s}\right) d s\right)\right]+(\log t) C_{6.3} \frac{\log (1+t)}{\sqrt{t}} \\
\rightarrow & c_{\phi}\left(Y_{0}\right) \text { as } t \rightarrow \infty
\end{aligned}
$$

by (8.5). Along with the previous liminf bound this proves (8.4).

To prove (8.3) we first establish a number of properties of

$$
\Phi(x, A)=E_{x}\left[\exp \left(-\int_{0}^{T_{A}} \phi\left(Y_{s}\right) d s\right)\right] .
$$

It is elementary that $0 \leq \Phi(x, A) \leq 1$ and that by recurrence, $\Phi(x, A) \rightarrow 0$ as $A \rightarrow \infty$. The next two results will show that $\Phi(x, A)$ is increasing in $|x|$.

Lemma 8.2. Let $N \in \mathbb{N}$. If $0=s_{0}<s_{1}<\cdots<s_{N}$ and $f: \mathbb{R}^{N+1} \rightarrow \mathbb{R}$ is bounded and $\uparrow$ in each coordinate then

$$
y \mapsto E_{y}\left[f\left(\left|Y_{s_{0}}\right|, \ldots,\left|Y_{s_{N}}\right|\right)\right] \text { is } \uparrow \text { in }|y| .
$$

Proof. Let $U, U_{1}, U_{2}, \ldots$ be iid rv's uniform on $B_{r}$, and let $S_{m}=U_{1}+\cdots+U_{m}$. The first step is to prove that if $N=1$ then for $m=1,2 \ldots$,

$$
E\left[f\left(|y|,\left|y+S_{m}\right|\right)\right] \text { is increasing in }|y| .
$$

Let $u \geq 0$, and define

$$
h_{u}(y)=P(|y+U| \leq u)=\frac{\left|B_{r} \cap B_{u}(-y)\right|}{\left|B_{r}\right|}=\frac{\left|B_{r} \cap B_{u}(y)\right|}{\left|B_{r}\right|} .
$$


It is easy to see that $h_{u}(y)$ is decreasing in $|y|$, so that $\left|y+S_{1}\right|$ is stochastically increasing in $|y|$, which proves (8.10) for $m=1$. Now suppose $m=2$, and consider

$$
P\left(\left|y+S_{2}\right| \leq u\right)=P\left(\left|y+S_{1}+U_{2}\right| \leq u\right)=E\left[h_{u}\left(y+S_{1}\right)\right] .
$$

Clearly $h_{u}(y)$ depends only on $|y|$, and having established it is decreasing in $|y|$, the $m=1$ case of (8.10) implies that $E\left[h_{u}\left(y+S_{2}\right)\right]$ is decreasing in $|y|$, which shows $\left|y+S_{2}\right|$ is stochastically increasing in $|y|$, proving (8.10) for $m=2$. The general inductive step for (8.10) is similar.

Consider next, the $N=1$ case of (8.9). With $\lambda=2 \rho\left|B_{r}\right|$,

$$
E_{y_{0}}\left[f\left(\left|Y_{s_{0}}\right|,\left|Y_{s_{1}}\right|\right)\right]=\sum_{m=0}^{\infty} e^{-\lambda s_{1}} \frac{\left(\lambda s_{1}\right)^{m}}{m !} E\left[f\left(\left|y_{0}\right|,\left|y_{0}+S_{2 m}\right|\right)\right]
$$

By (8.10), this shows $E_{y_{0}}\left[f\left(\left|Y_{s_{0}}\right|,\left|Y_{s_{1}}\right|\right)\right]$ is increasing in $\left|y_{0}\right|$, proving (8.9) for $N=1$. Now suppose $N>1$, let $\left(Y_{t}^{\prime}\right)$ under $P^{\prime}$ be an independent copy of $\left(Y_{t}\right)$, and define $\tilde{f}: \mathbb{R}^{N} \rightarrow \mathbb{R}$ by

$$
\tilde{f}\left(\left|y_{0}\right|,\left|y_{1}\right|, \ldots,\left|y_{N-1}\right|\right)=E_{y_{N-1}}^{\prime}\left[f\left(\left|y_{0}\right|, \ldots,\left|y_{N-1}\right|,\left|Y_{s_{N}-s_{N-1}}^{\prime}\right|\right)\right] .
$$

Then $\tilde{f}$ is increasing in $\left|y_{0}\right|, \ldots,\left|y_{N-2}\right|$ by definition, and is increasing in $\left|y_{N-1}\right|$ by the $N=1$ case of (8.9) just established. By the Markov property applied at time $s_{N-1}$,

$$
\begin{aligned}
E_{y_{0}}\left[f\left(\left|Y_{s_{0}}\right|, \ldots,\left|Y_{s_{N-1}}\right|,\left|Y_{s_{N}}\right|\right)\right] & =E_{y_{0}}\left[E_{Y_{s_{N-1}}}^{\prime}\left[f\left(\left|Y_{s_{0}}\right|, \ldots,\left|Y_{s_{N-1}}\right|,\left|Y_{s_{N}-s_{N-1}}^{\prime}\right|\right)\right]\right] \\
& =E_{y_{0}}\left[\tilde{f}\left(\left|Y_{s_{0}}\right|, \ldots,\left|Y_{s_{N-1}}\right|\right)\right] .
\end{aligned}
$$

This provides the inductive step to complete the proof of (8.9) for general $N$.

Lemma 8.3. Let $g:(0, \infty) \rightarrow[0, \infty)$ be continuous and $\downarrow$. Then for all $A, t \in[0, \infty]$,

$$
y \mapsto E_{y}\left[\exp \left(-\int_{0}^{T_{A} \wedge t} g\left(\left|Y_{s}\right|\right) d s\right)\right] \text { is } \uparrow \text { in }|y| .
$$

In particular, $x \rightarrow \Phi(x, A)$ is increasing in $|x|$.

Proof. By monotone convergence, we may assume $A, t$ are finite and $g$ is bounded. Let $g(0)=\lim _{s \downarrow 0} g(s)$. For $N \in \mathbb{N}$ let $M_{N} \in \mathbb{N}$ and $0=s_{0}^{N}<s_{1}^{N}<\cdots<s_{M_{N}}^{N}=t$ satisfy $s_{i+1}^{N}-s_{i}^{N}<2^{-N}$ for $0 \leq i<M_{N}$, and define

$$
\tau^{N}=\min \left\{s_{i}^{N}:\left|Y_{s_{i}^{N}}\right|>A\right\} \wedge t .
$$

By right-continuity of $\left|Y_{s}\right|, \tau^{N} \downarrow T_{A}$ a.s. as $N \rightarrow \infty$. By continuity of $g$ on $[0, \infty)$ and dominated convergence,

$$
\begin{aligned}
E_{y}\left[\operatorname { e x p } \left(-\int_{0}^{T_{A} \wedge t}\right.\right. & \left.\left.g\left(\left|Y_{s}\right|\right) d s\right)\right] \\
& =\lim _{N \rightarrow \infty} E_{y}\left[\exp \left(-\sum_{i=1}^{M_{N}-1} 1\left\{s_{i}^{N}<\tau^{N}\right\} g\left(\left|Y_{s_{i}^{N}}\right|\right)\left(s_{i+1}^{N}-s_{i}^{N}\right)\right)\right] \\
& =\lim _{N \rightarrow \infty} E_{y}\left[\prod_{i=0}^{M_{N}-1} G_{i}^{N}\left(\left|Y_{s_{0}^{N}}\right|, \ldots,\left|Y_{s_{i}^{N}}\right|\right)\right]
\end{aligned}
$$

where

$$
G_{i}^{N}\left(\left|Y_{s_{0}^{N}}\right|, \ldots,\left|Y_{s_{i}^{N}}\right|\right)=\exp \left(-1\left\{s_{i}^{N}<\tau^{N}\right\} g\left(\left|Y_{s_{i}^{N}}\right|\right)\left(s_{i+1}^{N}-s_{i}^{N}\right)\right) .
$$

It is easy to check that $G_{i}^{N}$ is increasing in each of its variables, and hence applying Lemma 8.2 to their product, $E_{y}\left[\prod_{i=0}^{M_{N}-1} G_{i}^{N}\left(\left|Y_{s_{0}}\right|, \ldots,\left|Y_{s_{i}}\right|\right)\right]$ is increasing in $|y|$. The result (8.13) now follows from the above. 


\section{Convergence of SLFV to SBM}

A consequence of the strong Markov property we will use repeatedly is

$$
\Phi(x, A)=P_{x}\left(T_{A}<t_{a}\right)+E_{x}\left[1\left\{t_{a}<T_{A}\right\} \Phi\left(Y_{t_{a}}, A\right)\right] \text { if } 2 r<a<|x|<A .
$$

Lemma 8.4. There exists $C_{8.15}=C_{8.15}(r)>1$ such that for all $k \geq 2$ and $0<|x| \leq k<A$,

$$
\Phi(x, A) \leq C_{8.15} \frac{\log k}{\log \left(A^{2}\right)} .
$$

Proof. By the monotonicity in Lemma 8.3, it suffices to prove (8.15) for $x=x_{k}=(k, 0)$. Assume additionally $k>6 r \vee r^{-1}$ and $A>r^{2}$. By (8.14), $\left|Y_{t_{3 r}}\right| \leq 3 r$, and monotonicity, we have

$$
\begin{aligned}
\Phi\left(x_{k}, A\right) & =P_{x_{k}}\left(T_{A}<t_{3 r}\right)+E_{x_{k}}\left[1\left\{t_{3 r}<T_{A}\right\} \Phi\left(Y_{t_{3 r}}, A\right)\right] \\
& \leq P_{x_{k}}\left(T_{A}<t_{3 r}\right)+P_{x_{k}}\left(t_{3 r}<T_{A}\right) \Phi((3 r, 0), A) .
\end{aligned}
$$

Using the strong Markov property at time $T_{4 r}$, and noting $\left|Y_{T_{4 r}}\right| \leq 6 r \leq\left|x_{k}\right|<A$, we again have from Lemma 8.3,

$$
\Phi((3 r, 0), A)=E_{(3 r, 0)}\left[\exp \left(-\int_{0}^{T_{4 r}} \phi\left(Y_{s}\right) d s\right) \Phi\left(Y_{T_{4 r}}, A\right)\right] \leq \alpha(r) \Phi\left(x_{k}, A\right),
$$

where we have set $\alpha(r)=\Phi\left(x_{3 r}, 4 r\right)<1$. Insert this into (8.16) and rearrange to conclude

$$
\Phi\left(x_{k}, A\right) \leq \frac{P_{x_{k}}\left(T_{A}<t_{3 r}\right)}{1-\alpha(r)} .
$$

By Lemma 5.3, taking $a=3 r$,

$$
P_{x_{k}}\left(T_{A}<t_{3 r}\right) \leq \frac{\log (k / r)}{\log (A / r)} \leq \frac{\log \left(k^{2}\right)}{\log (\sqrt{A})}=\frac{8 \log k}{\log \left(A^{2}\right)}
$$

where the second inequality uses $k>1 / r$ and $A>r^{2}$. In view of (8.17), letting $C=8 /(1-\alpha(r))$, we now have

$$
\Phi\left(x_{k}, A\right) \leq C \frac{\log k}{\log \left(A^{2}\right)}
$$

for all $k>6 r \vee r^{-1} \vee 2$ and $A>k \vee r^{2}$. It is easy to see that $C$ can be increased so that (8.19) will hold for all $k \geq 2$ and $A>k$, completing the proof of (8.15).

We will construct a coupling of the random walks $Y_{t}$ started at $x^{\prime} \neq x$ in order to obtain good bounds on the difference $\Phi\left(x^{\prime}, A\right)-\Phi(x, A)$. We start in discrete time. Let $\left\{U_{i}\right\}$ be iid r.v.'s which are uniformly distributed over $B_{r}$, and for $x^{\prime} \in H_{r}=\left\{\left(x_{1}, x_{2}\right): x_{1}>0\right\}$ define

$$
S_{n}^{x^{\prime}}=x^{\prime}+\sum_{i=1}^{n} U_{i}
$$

Let $\pi$ denote the reflection mapping $\pi\left(x_{1}, x_{2}\right)=\left(-x_{1}, x_{2}\right)$ and set $x=\pi\left(x^{\prime}\right)$. We will use a reflection coupling to define $\left(S_{n}^{x}: n \geq 0\right)$. Let $H_{\ell}=\left\{\left(x_{1}, x_{2}\right): x_{1} \leq 0\right\}$, , and define

$$
N_{c}=N_{c}^{x, x^{\prime}}=\min \left\{n \geq 1: S_{n}^{x^{\prime}} \in B_{r}\left(\pi\left(S_{n-1}^{x^{\prime}}\right)\right)\right\} .
$$

Lemma 8.5. $N_{c} \leq N_{\ell}:=\min \left\{n \geq 0: S_{n}^{x^{\prime}} \in H_{\ell}\right\}$ a.s., and so $S_{n}^{x^{\prime}} \in H_{r}$ for all $0 \leq n<N_{c}$ a.s. 
Proof. $S_{N_{\ell}}^{x^{\prime}} \in H_{\ell}$ and $\pi\left(S_{N_{\ell}-1}^{x^{\prime}}\right) \in H_{\ell}$ imply that

$$
\left|S_{N_{\ell}}^{x^{\prime}}-\pi\left(S_{N_{\ell}-1}^{x^{\prime}}\right)\right| \leq\left|\pi\left(S_{N_{\ell}}^{x^{\prime}}\right)-\pi\left(S_{N_{\ell}-1}^{x^{\prime}}\right)\right|=\left|S_{N_{\ell}}^{x^{\prime}}-S_{N_{\ell}-1}^{x^{\prime}}\right|<r .
$$

The result follows.

We now define $\left(S_{n}^{x}\right)_{n \geq 0}$ by

$$
S_{n}^{x}= \begin{cases}\pi\left(S_{n}^{x^{\prime}}\right) & \text { if } n<N_{c} \\ S_{n}^{x^{\prime}} & \text { if } n \geq N_{c} .\end{cases}
$$

Then $S_{0}^{x}=x$, and it follows from Lemma 8.5 that for $n<N_{c}, S_{n}^{x^{\prime}}$ is in $H_{r}$ and so $S_{n}^{x^{\prime}} \neq S_{n}^{x}$, which implies that

$$
N_{c}=\min \left\{n \geq 0: S_{n}^{x^{\prime}}=S_{n}^{x}\right\} .
$$

That is, $N_{c}$ is the coupling time of $\left(S_{n}^{x}\right)$ and $\left(S_{n}^{x^{\prime}}\right)$. If we let $\mathcal{F}_{n}^{S^{x^{\prime}}}=\sigma\left(S_{m}^{x^{\prime}}, m \leq n\right)$, then $N_{c}$ is an $\left(\mathcal{F}_{n}^{S^{x^{\prime}}}\right)$-stopping time, and $S^{x}$ is $\left(\mathcal{F}_{n}^{S^{x^{\prime}}}\right)$-adapted. We next show that $S_{n}^{x}$ is an $\left(\mathcal{F}_{n}^{S^{x^{\prime}}}\right)$-random walk starting at $x$ with step distribution $U_{1}$, as the notation suggests.

Lemma 8.6. For any Borel $A \subset \mathbb{R}^{2}, P\left(S_{n+1}^{x} \in A \mid \mathcal{F}_{n}^{S^{x^{\prime}}}\right)(\omega)=P\left(S_{n}^{x}(\omega)+U_{n+1} \in A\right)$ a.s.

Proof. This is obvious on $\left\{N_{c} \leq n\right\}$ (in $\mathcal{F}_{n}^{S^{x^{\prime}}}$ ) since then $S_{n}^{x}$ and $S_{n+1}^{x}$ equal $S_{n}^{x^{\prime}}$ and $S_{n+1}^{x^{\prime}}$, respectively. Suppose now that $N_{c}>n$, and define $\hat{B}=\hat{B}(\omega)=B_{r}\left(S_{n}^{x^{\prime}}\right) \cap B_{r}\left(\pi\left(S_{n}^{x^{\prime}}\right)\right)$, so that

$$
\pi(\hat{B})=\hat{B}
$$

and

$$
\hat{B} \subset B_{r}\left(S_{n}^{x}\right) .
$$

This last inclusion holds because $S_{n}^{x}=S_{n}^{x^{\prime}}$ or $\pi\left(S_{n}^{x^{\prime}}\right)$ for all $n$. $S_{n}^{x}$

For simplicity we will write $\mathcal{F}_{n}$ for $\mathcal{F}_{n}^{S^{x^{\prime}}}$ in the rest of this proof. By the definition of

$$
\begin{aligned}
& P\left(S_{n+1}^{x} \in A \mid \mathcal{F}_{n}\right) 1\left(N_{c}>n\right) \\
& =P\left(S_{n+1}^{x^{\prime}} \in B_{r}\left(\pi\left(S_{n}^{x^{\prime}}\right)\right), N_{c}>n, S_{n}^{x^{\prime}}+U_{n+1} \in A \mid \mathcal{F}_{n}\right) \\
& \quad+P\left(S_{n+1}^{x^{\prime}} \notin B_{r}\left(\pi\left(S_{n}^{x^{\prime}}\right)\right), N_{c}>n, \pi\left(S_{n+1}^{x^{\prime}}\right) \in A \mid \mathcal{F}_{n}\right) \\
& =P\left(S_{n+1}^{x^{\prime}} \in \hat{B} \cap A, N_{c}>n \mid \mathcal{F}_{n}\right)+P\left(S_{n+1}^{x^{\prime}} \notin \hat{B}, N_{c}>n, \pi\left(S_{n+1}^{x^{\prime}}\right) \in A \mid \mathcal{F}_{n}\right) \\
& \left.=P\left(\pi\left(S_{n+1}^{x^{\prime}}\right) \in \hat{B} \cap \pi(A), N_{c}>n \mid \mathcal{F}_{n}\right)+P\left(\pi\left(S_{n+1}^{x^{\prime}}\right) \in \hat{B}^{c} \cap A, N_{c}>n\right) \mid \mathcal{F}_{n}\right)(\text { by (8.22)) } \\
& =\left[P\left(S_{n}^{x}+\pi\left(U_{n+1}\right) \in \hat{B} \cap \pi(A) \mid \mathcal{F}_{n}\right)+P\left(S_{n}^{x}+\pi\left(U_{n+1}\right) \in \hat{B}^{c} \cap A \mid \mathcal{F}_{n}\right)\right] 1\left(N_{c}>n\right) .
\end{aligned}
$$

Next introduce the dependence on $\omega$ in the above, and use the fact that, conditionally on $\mathcal{F}_{n}, S_{n}^{x}(\omega)+\pi\left(U_{n+1}\right)$ is uniformly distributed over $B_{r}\left(S_{n}^{x}(\omega)\right)$ to see that if $|C|$ is the Lebesgue measure of $C$, then the above evaluated at $\omega$ is a.s. equal to

$$
\begin{aligned}
{[\mid} & \left.\left|\pi(A) \cap \hat{B}(\omega) \cap B_{r}\left(S_{n}^{x}(\omega)\right)\right|+\left|A \cap \hat{B}^{c}(\omega) \cap B_{r}\left(S_{n}^{x}(\omega)\right)\right|\right] 1\left(N_{c}(\omega)>n\right) /\left|B_{r}\right| \\
= & {\left[|A \cap \hat{B}(\omega)|+\left|A \cap \hat{B}^{c}(\omega) \cap B_{r}\left(S_{n}^{x}(\omega)\right)\right|\right] 1\left(N_{c}(\omega)>n\right) /\left|B_{r}\right| } \\
& \quad(\operatorname{by}|\pi(C)|=|C|,(8.22),(8.23)) \\
= & {\left[\left|A \cap \hat{B}(\omega) \cap B_{r}\left(S_{n}^{x}(\omega)\right)\right|+\left|A \cap \hat{B}^{c}(\omega) \cap B_{r}\left(S_{n}^{x}(\omega)\right)\right|\right] 1\left(N_{c}(\omega)>n\right) /\left|B_{r}\right| \quad \text { (by (8.23)) } } \\
= & \mid A \cap B_{r}\left(S_{n}^{x}(\omega)\left|1\left(N_{c}(\omega)>n\right) /\right| B_{r} \mid\right. \\
= & P\left(S_{n}^{x}(\omega)+U_{n+1} \in A\right) 1\left(N_{c}(\omega)>n\right) .
\end{aligned}
$$

The result follows. 
Let $S_{n}=\left(S_{n}^{(1)}, S_{n}^{(2)}\right)$ denote a copy of the random walk starting at $x^{\prime}$ under $P_{x^{\prime}}$.

Lemma 8.7. There is a constant $C_{8.7}$ so that for all $x^{\prime}$ in the positive $x_{1}$-axis and all $n \in \mathbb{N}$,

$$
P\left(N_{c}^{x, x^{\prime}} \geq n\right) \leq \frac{C_{8.7}}{\sqrt{n}}\left(1+\frac{\left|x^{\prime}\right|}{2 r}\right) .
$$

Proof. Use Lemma 8.5 and then the reflection principle to see that

$$
\begin{aligned}
P\left(N_{c}^{x, x^{\prime}} \geq n\right) \leq P_{x^{\prime}}\left(N_{\ell} \geq n\right) & =1-P_{x^{\prime}}\left(N_{\ell}<n\right) \\
& =1-2 P_{x^{\prime}}\left(S_{n}^{(1)}<S_{N_{\ell}}^{(1)}, N_{\ell}<n\right) \\
& \leq 1-2 P_{0}\left(S_{n}^{(1)}<-\left|x^{\prime}\right|-r\right) \\
& =P_{0}\left(\left|S_{n}^{(1)}\right| \leq r+\left|x^{\prime}\right|\right) .
\end{aligned}
$$

The step distribution of $\left(S_{n}^{(1)}\right)$ has density $f(u)=2 \sqrt{r^{2}-u^{2}} /\left|B_{r}\right| \leq 1 / r$ on $[-r, r]$. It follows from the $d=1$ version of (5.6) applied to random variables with this distribution that for a constant $C=C(r)$,

$$
P_{0}\left(\left|S_{n}^{(1)}\right| \leq r+\left|x^{\prime}\right|\right) \leq C \frac{2\left(r+\left|x^{\prime}\right|\right)}{\sqrt{n}} \leq \frac{4 C r}{\sqrt{n}}\left(1+\frac{\left|x^{\prime}\right|}{2 r}\right),
$$

so we are done.

We now use translation invariance to extend the above to points $x, x^{\prime} \in\left\{\left(x_{1}, 0\right)\right.$ : $\left.x_{1} \geq 0\right\}$ such that $0 \leq|x|<\left|x^{\prime}\right|$, where now $\frac{x+x^{\prime}}{2}=(m, 0)$ plays the role of the origin, $H_{\ell}^{m}=\left\{x: x_{1} \leq m\right\}$, and $\pi^{m}$ is reflection in the plane $\left\{x_{1}=m\right\}$. So we define

$$
N_{c}^{x, x^{\prime}}=N_{c}=\min \left\{n \geq 1: S_{n}^{x^{\prime}} \in B_{r}\left(\pi^{m}\left(S_{n-1}^{x^{\prime}}\right)\right)\right\} \leq N_{\ell}^{x^{\prime}}=\min \left\{n \geq 0: S_{n}^{x^{\prime}} \in H_{\ell}^{m}\right\},
$$

where the inequality is by Lemma 8.5 , and

$$
S_{n}^{x}= \begin{cases}\pi^{m}\left(S_{n}^{x^{\prime}}\right) & \text { if } n<N_{c} \\ S_{n}^{x^{\prime}} & \text { if } n \geq N_{c}\end{cases}
$$

The above results imply that both $S^{x}$ and $S^{x^{\prime}}$ are $\left(\mathcal{F}_{n}^{S^{x^{\prime}}}\right)$-random walks with step distribution $U_{1}$,

$$
N_{c}^{x, x^{\prime}}=\min \left\{n \geq 0: S_{n}^{x}=S_{n}^{x^{\prime}}\right\}
$$

is their coupling time, and

$$
P\left(N_{c}^{x, x^{\prime}} \geq n\right) \leq \frac{C_{8.7}}{\sqrt{n}}\left(1+\frac{\left|x^{\prime}-x\right|}{2 r}\right) .
$$

Next define coupled copies of the discrete time random walk with step distribution $U_{1}+U_{2}$ by $\hat{Y}_{n}^{x}=S_{2 n}^{x}$ and $\hat{Y}_{n}^{x^{\prime}}=S_{2 n}^{x^{\prime}}$, and also set $\hat{\mathcal{F}}_{n}^{x^{\prime}}=\mathcal{F}_{2 n}^{S^{x^{\prime}}}$. We will write $\hat{\mathcal{F}}_{n}$ for $\hat{\mathcal{F}}_{n}^{x^{\prime}}$ if there is no ambiguity. Then it follows from Lemma 8.6 that both $\hat{Y}_{n}^{x}$ and $\hat{Y}_{n}^{x^{\prime}}$ are $\left(\hat{\mathcal{F}}_{n}\right)$-random walks with step distribution $U_{1}+U_{2}$, that is, they are $\left(\hat{\mathcal{F}}_{n}\right)$-adapted and

$$
P\left(\hat{Y}_{n+1}^{x} \in A \mid \hat{\mathcal{F}}_{n}\right)(\omega)=P\left(\hat{Y}_{n}^{x}(\omega)+U_{1}+U_{2} \in A\right) \text { a.s. }
$$

and similarly for $\hat{Y}^{x^{\prime}}$. It follows easily from (8.26) that

$$
\hat{N}_{c}^{x, x^{\prime}}:=\min \left\{n \geq 0: \hat{Y}_{n}^{x}=\hat{Y}_{n}^{x^{\prime}}\right\}=\left\lceil\frac{N_{c}^{x, x^{\prime}}}{2}\right\rceil .
$$


Next use (8.25) and the fact that $2 n \geq N_{c}^{x, x^{\prime}}$ iff $n \geq\left\lceil\frac{N_{c}^{x, x^{\prime}}}{2}\right\rceil$ to conclude that

$$
\hat{Y}_{n}^{x}= \begin{cases}\pi^{m}\left(\hat{Y}_{n}^{x^{\prime}}\right) & \text { if } n<\hat{N}_{c}^{x, x^{\prime}} \\ \hat{Y}_{n}^{x^{\prime}} & \text { if } n \geq \hat{N}_{c}^{x, x^{\prime}}\end{cases}
$$

Letting $\hat{Y}_{n}^{x^{\prime},(1)}$ be the first coordinate of $\hat{Y}_{n}^{x^{\prime}}$, define

$$
\hat{N}^{x^{\prime}}(m)=\min \left\{n \geq 0: \hat{Y}_{n}^{x^{\prime},(1)} \leq m\right\} .
$$

Lemma 8.8. With $x, x^{\prime}, m$ as above, $\hat{N}_{c}^{x, x^{\prime}} \leq \hat{N}^{x^{\prime}}(m)$ a.s.

Proof. It follows from Lemma 8.5 that for all $n, S_{2 n}^{x} \neq S_{2 n}^{x^{\prime}}$ implies that $S_{2 n}^{(1), x^{\prime}}>m$. This shows that $n<\hat{N}_{c}^{x, x^{\prime}}$ implies $n<\hat{N}^{x^{\prime}}(m)$ which clearly gives the required result.

Lemma 8.9. With $x, x^{\prime}, m$ as above, and for all $n \in \mathbb{N}$,

$$
P\left(\hat{N}_{c}^{x, x^{\prime}} \geq n\right) \leq C_{8.7} n^{-1 / 2}\left(1+\frac{\left|x^{\prime}-x\right|}{2 r}\right) .
$$

Proof. By (8.29)

$$
P\left(\hat{N}_{c}^{x, x^{\prime}} \geq n\right)=P\left(\left\lceil\frac{N_{c}^{x, x^{\prime}}}{2}\right\rceil \geq n\right) \leq P\left(N_{c}^{x, x^{\prime}} \geq 2 n-1\right) .
$$

The result follows from (8.27).

We move now to the continuous time random walks. Let $N(t)$ be an independent Poisson process with rate $\lambda=2 \rho\left|B_{r}\right|$ and jump time sequence $\left(s_{n}\right)_{n \in \mathbb{Z}_{+}}$, i.e., $s_{n}=\inf \{t \geq$ $\left.0: N_{t}=n\right\}$. For $K>0$ put $x^{\prime}=(K+2 r, 0)$, and let $x \in[K, K+2 r) \times\{0\}$. Define coupled continuous time rate $\lambda$ random walks with step distribution $U_{1}+U_{2}$, starting at $x^{\prime}$ and $x$, respectively, by

$$
Y_{t}^{x^{\prime}}=\hat{Y}_{N_{t}}^{x^{\prime}}=\hat{Y}_{n}^{x^{\prime}} \text { if } s_{n} \leq t<s_{n+1}
$$

and

$$
Y_{t}^{x}=\hat{Y}_{N_{t}}^{x}=\hat{Y}_{n}^{x} \text { if } s_{n} \leq t<s_{n+1} .
$$

The coupling time of these random walks is

$$
\begin{aligned}
S_{c}^{x, x^{\prime}}:=\inf \left\{t \geq 0: Y_{t}^{x}=Y_{t}^{x^{\prime}}\right\} & =\inf \left\{t \geq 0: \hat{Y}_{N_{t}}^{x}=\hat{Y}_{N_{t}}^{x^{\prime}}\right\} \\
& =\inf \left\{t \geq 0: N_{t}=\hat{N}_{c}^{x, x^{\prime}}\right\}=s_{\hat{N}_{c}^{x, x^{\prime}}}
\end{aligned}
$$

Note that $t<S_{c}^{x, x^{\prime}}=s_{\hat{N}_{c}^{x, x^{\prime}}}$ iff $N_{t}<\hat{N}_{c}^{x, x^{\prime}}$, and so by setting $n=N_{t}$ in (8.30), we have

$$
Y_{t}^{x}= \begin{cases}\pi^{m}\left(Y_{t}^{x^{\prime}}\right) & \text { if } t<S_{c}^{x, x^{\prime}} \\ Y_{t}^{x^{\prime}} & \text { if } t \geq S_{c}^{x, x^{\prime}} .\end{cases}
$$

Let $\mathcal{F}_{t}$ be the right-continuous filtration generated by $\left(Y^{x}, Y^{x^{\prime}}, N\right)$, and let $Y_{t}$ (respectively $\hat{Y}_{n}$ ) denote a generic rate $\lambda$ continuous time (respectively, discrete time) random walk with step distribution $U_{1}+U_{2}$, starting at 0 under $P_{0}$.

Lemma 8.10. (a) Both $Y^{x}$ and $Y^{x^{\prime}}$ are rate $\lambda$ continuous time $\left(\mathcal{F}_{t}\right)$-random walks (and $\left(\mathcal{F}_{t}\right)$-strong Markov processes) with jump distribution $U_{1}+U_{2}$. That is for $y=x$ or $x^{\prime}$, $t>0$, and any a.s. finite $\left(\mathcal{F}_{t}\right)$-stopping time $S$,

$$
P\left(Y_{S+t}^{y} \in A \mid \mathcal{F}_{S}\right)(\omega)=P_{0}\left(Y_{S}^{y}(\omega)+Y_{t} \in A\right) \text { a.s. for any Borel } A \subset \mathbb{R}^{2} .
$$

(b) $S_{c}^{x, x^{\prime}}$ is an $\left(\mathcal{F}_{t}\right)$-stopping time. 
Proof. (b) is obvious from the definition of $S_{c}^{x, x^{\prime}}$.

(a) This is an easy and standard consequence of (8.28).

For $y=x$ or $x^{\prime}$ and $2 r \leq \delta<A$ we let

$$
t_{\delta}^{y}=\inf \left\{t \geq 0:\left|Y_{s}^{y}\right| \leq \delta\right\}, \quad T_{A}^{y}=\inf \left\{t \geq 0:\left|Y_{t}^{y}\right| \geq A\right\},
$$

and also set

$$
t_{\delta}^{x, x^{\prime}}=t_{\delta}^{x} \wedge t_{\delta}^{x^{\prime}}, \quad T_{A}^{x, x^{\prime}}=T_{A}^{x} \wedge T_{A}^{x^{\prime}} .
$$

We define $\hat{t}_{\delta}^{y}, \hat{T}_{A}^{y}, \hat{t}_{\delta}^{x, x^{\prime}}$, and $\hat{T}_{A}^{x, x^{\prime}}$ in a similar way, using the discrete time random walks $\hat{Y}^{x}, \hat{Y}^{x^{\prime}}$, for example,

$$
\hat{t}_{\delta}^{y}=\min \left\{n \geq 0:\left|\hat{Y}_{n}^{y}\right| \leq \delta\right\} .
$$

Lemma 8.11. Let $K>3 r$ and $x^{\prime}=(K+2 r, 0)$.

(a) For all $x \in[K, K+2 r) \times\{0\}, t_{3 r}^{x^{\prime}} \geq S_{c}^{x, x^{\prime}} \vee t_{3 r}^{x}$.

(b) For all $x \in[K, K+2 r) \times\{0\}$, if $t_{3 r}^{x}<S_{c}^{x, x^{\prime}}$, then $\left|Y_{t_{3 r}^{x}}^{x^{\prime}}\right| \leq 2 K+10 r$.

(c) For $\varepsilon \in(0,1)$ and $\delta \geq 3 r$ there is a constant $C_{8.34}=C_{8.34}(\delta, \varepsilon)>0$ such that for all $K>\delta \vee 1$,

$$
\inf _{x \in[K, K+2 r) \times\{0\}} P\left(S_{c}^{x, x^{\prime}}<t_{\delta}^{x, x^{\prime}} \wedge T_{2 K}^{x, x^{\prime}}\right) \geq 1-\frac{C_{8.34}}{K^{1-\varepsilon}} .
$$

Proof. (a) First consider the discrete time walks. For $x \in[K, K+2 r) \times\{0\}$, we have $m=\frac{\left|x+x^{\prime}\right|}{2}>K>3 r$. This shows that $B_{3 r} \subset\left\{x_{1}<m\right\}$ and so $\hat{Y}^{x^{\prime}}$ must first enter $\left\{x_{1} \leq m\right\}$ before it can enter $B_{3 r}$. That is, $\hat{t}_{3 r}^{x^{\prime}} \geq \hat{N}^{x^{\prime}}(m)$. Therefore by Lemma 8.8, $\hat{N}_{c}^{x, x^{\prime}} \leq \hat{t}_{3 r}^{x^{\prime}}$ and hence

$$
S_{c}^{x, x^{\prime}}=s_{\hat{N}_{c}^{x, x^{\prime}}} \leq s_{\hat{t}_{3 r}^{x^{\prime}}}=t_{3 r}^{x^{\prime}} .
$$

Since the random walks must couple before $Y^{x^{\prime}}$ can enter $B_{3 r}$, we also have $t_{3 r}^{x} \leq t_{3 r}^{x^{\prime}}$ and (a) follows.

(b) Let $x$ and $m$ be as in (a). If $t_{3 r}^{x}<S_{c}^{x, x^{\prime}}$, then by the coupling definition (8.32),

$$
Y_{t_{3 r}^{x}}^{x}=\pi^{m}\left(Y_{t_{3 r}^{x}}^{x^{\prime}}\right) .
$$

For any $a=\left(a_{1}, a_{2}\right) \in B_{3 r},\left|\pi^{m}(a)\right|=\left|\left(2 m-a_{1}, a_{2}\right)\right| \leq 2 m+3 r+3 r \leq 2 K+10 r$, so by (8.35),

This proves (b).

$$
\left|Y_{t_{3 r}^{x}}^{x^{\prime}}\right|=\left|\pi^{m}\left(Y_{t_{3 r}^{x}}^{x}\right)\right| \leq 2 K+10 r
$$

(c) Let $\hat{Y}^{(1)}$ be the first coordinate of $\hat{Y}$, and let $x, x^{\prime}, m$ be as above, with $K>\delta \geq 3 r$, so that $\delta<|x| \leq\left|x^{\prime}\right|<2 K$. Let $n=\left\lceil K^{2-2 \varepsilon}\right\rceil$. Then, using Lemma 8.9 for the second inequality and symmetry for the second to last inequality, we have

$$
\begin{aligned}
P\left(\hat{N}_{c}^{x, x^{\prime}}<\hat{t}_{\delta}^{x, x^{\prime}} \wedge \hat{T}_{2 K}^{x, x^{\prime}}\right) & \\
\geq & P\left(\hat{N}_{c}^{x, x^{\prime}} \leq n\right)-P\left(\hat{t}_{\delta}^{x^{\prime}} \wedge \hat{T}_{2 K}^{x^{\prime}} \leq n\right)-P\left(\hat{t}_{\delta}^{x} \wedge \hat{T}_{2 K}^{x} \leq n\right) \\
\geq & 1-\frac{C_{8.7}}{\sqrt{n}}\left(1+\frac{\left|x^{\prime}-x\right|}{2 r}\right)-P_{0}\left(\max _{k \leq n}\left|\hat{Y}_{k}\right| \geq(K-2 r) \wedge(K+2 r-\delta)\right) \\
& \quad-P_{0}\left(\max _{k \leq n}\left|\hat{Y}_{k}\right| \geq(K-2 r) \wedge(K-\delta)\right) \\
\geq & 1-\frac{2 C_{8.7}}{\sqrt{n}}-2 P_{0}\left(\max _{k \leq n}\left|\hat{Y}_{k}\right| \geq K-\delta\right) \\
\geq & 1-\frac{C}{K^{1-\varepsilon}}-4 P_{0}\left(\max _{k \leq n}\left|\hat{Y}_{k}^{(1)}\right| \geq \frac{K-\delta}{\sqrt{2}}\right) \\
\geq & 1-\frac{C}{K^{1-\varepsilon}}-4 P_{0}\left(\max _{k \leq n}\left|\hat{Y}_{k}^{(1)}\right| \geq K / 2\right)
\end{aligned}
$$


provided $K$ is larger than some $K_{0}(\delta)>0$. We recall Theorem 21.1 in [4], which in the present context implies

$$
E_{0}\left[\max _{k \leq n}\left|\hat{Y}_{k}^{(1)}\right|^{p}\right] \leq c_{p}\left[\left(n E\left[\left|\bar{U}^{(1)}\right|^{2}\right]\right)^{p / 2}+(2 r)^{p}\right] \leq C_{p} n^{p / 2}
$$

for a constant $C_{p}>0$. By Markov's inequality,

$$
P_{0}\left(\max _{k \leq n}\left|\hat{Y}_{k}^{(1)}\right| \geq K / 2\right) \leq \frac{C_{p} n^{p / 2}}{(K / 2)^{p}} \leq \frac{C}{K^{p \varepsilon}} .
$$

If we take $p=p_{0}(\varepsilon)$ large enough so that $K^{p \varepsilon}>K^{1-\varepsilon}$, substituting into (8.36) we obtain for a constant $C>0$ depending on $\varepsilon$,

$$
P\left(\hat{N}_{c}^{x, x^{\prime}}<\hat{t}_{\delta}^{x, x^{\prime}} \wedge \hat{T}_{2 K}^{x, x^{\prime}}\right) \geq 1-\frac{C}{K^{1-\varepsilon}},
$$

provided $K \geq K_{0}(\delta)$. Multiplication of $C$ by a large enough constant depending on $\delta$ allows us to remove the restriction $K>K_{0}(\delta)$. That is, for some $C_{8.34}(\delta, \varepsilon)>0$,

$$
\inf _{x \in[K, K+2 r) \times\{0\}} P\left(\hat{N}_{c}^{x, x^{\prime}}<\hat{t}_{\delta}^{x, x^{\prime}} \wedge \hat{T}_{2 K}^{x, x^{\prime}}\right) \geq 1-\frac{C_{8.34}(\delta, \varepsilon)}{K^{1-\varepsilon}}
$$

for all $K>\delta \vee 1$.

The corresponding inequality for the continuous time walks follows at once. First, note that

$$
t_{\delta}^{x}=\inf \left\{t:\left|\hat{Y}_{N_{t}}^{x}\right| \leq \delta\right\}=\inf \left\{t: N_{t}=\hat{t}_{\delta}^{x}\right\}=s_{\hat{t}_{\delta}^{x}},
$$

and similarly for the other hitting times. So in view of (8.31), for every $x \in[K, K+2 r) \times$ $\{0\}$,

$$
\begin{aligned}
P\left(T_{c}^{x, x^{\prime}}<t_{\delta}^{x, x^{\prime}} \wedge T_{2 K}^{x, x^{\prime}}\right) & =P\left(s_{\hat{N}_{c}^{x, x^{\prime}}}<s_{\hat{t}_{\delta}^{x, x^{\prime}}} \wedge s_{\hat{T}_{2 K}^{x, x^{\prime}}}\right) \\
& =P\left(\hat{N}_{c}^{x, x^{\prime}}<\hat{t}_{\delta}^{x, x^{\prime}} \wedge T_{2 K}^{x, x^{\prime}}\right) .
\end{aligned}
$$

So (c) is now immediate from (8.38).

Lemma 8.12. There is a constant $C_{8.39}=C_{8.39}(r, \phi)>0$ such that for $K>5 r \vee 2$, $A>2 K+2 r, x, x^{\prime} \in[K, K+2 r] \times\{0\}$ with $|x| \leq\left|x^{\prime}\right|$, and $\varepsilon_{K}=(\log K) / \sqrt{K}$,

$$
0 \leq \Phi\left(x^{\prime}, A\right)-\Phi(x, A) \leq C_{8.39} \frac{\varepsilon_{K}}{\log \left(A^{2}\right)} .
$$

Proof. The first inequality in (8.39) follows from monotonicity (Lemma 8.3). For the second, it suffices to take $x^{\prime}=(K+2 r, 0)$ and $x \in[K, K+2 r) \times\{0\}$. Recall the times $t_{\delta}^{x, x^{\prime}}=t_{\delta}^{x} \wedge t_{\delta}^{x^{\prime}}, T_{A}^{x, x^{\prime}}=T_{A}^{x} \wedge T_{A}^{x^{\prime}}$, etc. for the coupled walks $\left(Y^{x}, Y^{x^{\prime}}\right)$, and write $S_{c}$ for the coupling time $S_{c}^{x, x^{\prime}}$. By Lemma 8.11(a),

$$
t_{3 r}^{x, x^{\prime}}=t_{3 r}^{x}
$$

If $S_{c} \leq t_{3 r}^{x} \wedge T_{A}^{x, x^{\prime}}$, then

As a consequence,

$$
\int_{0}^{T_{A}^{x^{\prime}}} \phi\left(Y_{s}^{x^{\prime}}\right) d s=\int_{0}^{T_{A}^{x}} \phi\left(Y_{s}^{x}\right) d s .
$$

$$
\begin{aligned}
\Delta\left(x, x^{\prime}, A\right) & :=\Phi\left(x^{\prime}, A\right)-\Phi(x, A) \\
& =E\left[\exp \left(-\int_{0}^{T_{A}^{x^{\prime}}} \phi\left(Y_{s}^{x^{\prime}}\right) d s\right)-\exp \left(-\int_{0}^{T_{A}^{x}} \phi\left(Y_{s}^{x}\right) d s\right)\right] \\
& \leq E\left[1\left\{S_{c}>t_{3 r}^{x} \wedge T_{A}^{x, x^{\prime}}\right\} \exp \left(-\int_{0}^{T_{A}^{x^{\prime}}} \phi\left(Y_{s}^{x^{\prime}}\right) d s\right)\right] .
\end{aligned}
$$




\section{Convergence of SLFV to SBM}

We now introduce

$$
\begin{aligned}
& \Delta_{1}\left(x, x^{\prime}, A\right)=E\left[1\left\{T_{A}^{x, x^{\prime}}<S_{c}, T_{A}^{x, x^{\prime}} \leq t_{3 r}^{x}\right\} \exp \left(-\int_{0}^{T_{A}^{x^{\prime}}} \phi\left(\left|Y_{s}^{x^{\prime}}\right|\right) d s\right)\right], \\
& \Delta_{2}\left(x, x^{\prime}, A\right)=E\left[1\left\{t_{3 r}^{x}<S_{c}, t_{3 r}^{x}<T_{A}^{x, x^{\prime}}\right\} \exp \left(-\int_{0}^{T_{A}^{x^{\prime}}} \phi\left(\left|Y_{s}^{x^{\prime}}\right|\right) d s\right)\right],
\end{aligned}
$$

so that $\Delta=\Delta_{1}+\Delta_{2}$, and bound $\Delta_{1}, \Delta_{2}$ separately. For $\Delta_{1}$, using (8.40) and $T_{A}^{x^{\prime}} \neq t_{3 r}^{x^{\prime}}$,

$$
\Delta_{1}\left(x, x^{\prime}, A\right) \leq P\left(T_{A}^{x}<S_{c} \wedge t_{3 r}^{x}\right)+P\left(T_{A}^{x^{\prime}}<S_{c} \wedge t_{3 r}^{x^{\prime}}\right) .
$$

It suffices to consider the first term, as the second follows in the same way. By the strong Markov property

$$
P\left(T_{A}^{x}<S_{c} \wedge t_{3 r}^{x}\right) \leq E\left[1\left\{T_{2 K}^{x}<S_{c}\right\} P_{Y_{T_{2 K}^{x}}^{x}}\left(T_{A}<t_{3 r}\right)\right] .
$$

Now taking $a=3 r$ in Lemma 5.3, and noting that $3 r<2 K \leq\left|Y_{T_{2 K}^{x}}^{x}\right| \leq 2 K+2 r<A$, we have

$$
P_{Y_{2 K}^{x}}\left(T_{A}<t_{3 r}\right) \leq \frac{\log \left(\left|Y_{T_{2 K}^{x}}^{x}\right|\right)-\log r}{\log A-\log r} \leq \frac{\log \left(\frac{2 K}{r}+2\right)}{\log (A / r)} \text { a.s. }
$$

Choose $K_{0}$ large enough so that $K>K_{0}$ implies $K^{2}>\frac{2 K}{r}+2$. If, in addition we have $K>K_{0}$ and $A>r^{2}$, then

$$
P_{Y_{2 K}^{x}}\left(T_{A}<t_{3 r}\right) \leq \frac{\log \left(K^{2}\right)}{\log \sqrt{A}}=\frac{8 \log K}{\log \left(A^{2}\right)} .
$$

By replacing 8 with a sufficiently large constant $C$ we may drop the additional conditions $K>K_{0}$ and $A>r^{2}$, and so obtain for all $K>5 r \wedge 2$ and $A>2 K+2 r$,

$$
P_{Y_{T_{2 K}}^{x}}\left(T_{A}<T_{3 r}\right) \leq C \frac{\log K}{\log \left(A^{2}\right)} .
$$

Plug this bound into (8.42) and use the coupling bound Lemma 8.11(c) with $\delta=3 r, \varepsilon=$ $1 / 2$ to obtain

$$
P\left(T_{A}^{x}<S_{c} \wedge t_{3 r}^{x}\right) \leq C \frac{\log K}{\log \left(A^{2}\right)} P\left(T_{2 K}^{x}<S_{c}\right) \leq C_{8.34}\left(3 r, \frac{1}{2}\right) C \frac{\log K}{\sqrt{K} \log \left(A^{2}\right)} .
$$

The above and (8.41) imply

$$
\Delta_{1}\left(x, x^{\prime}, A\right) \leq C \frac{\log K}{\sqrt{K} \log \left(A^{2}\right)} .
$$

Now consider $\Delta_{2}$. Recalling from (8.40) that $t_{3 r}^{x} \leq t_{3 r}^{x^{\prime}}, \Delta_{2}$ is bounded by the sum of

$$
\begin{aligned}
& \Delta_{2 a}\left(x, x^{\prime}, A\right)=E\left[1\left\{t_{3 r}^{x}<S_{c}, t_{3 r}^{x} \leq t_{3 r}^{x^{\prime}}<T_{A}^{x^{\prime}}\right\} \exp \left(-\int_{0}^{T_{A}^{x^{\prime}}} \phi\left(\left|Y_{s}^{x^{\prime}}\right|\right) d s\right)\right], \\
& \Delta_{2 b}\left(x, x^{\prime}, A\right)=E\left[1\left\{t_{3 r}^{x}<S_{c}, t_{3 r}^{x}<T_{A}^{x^{\prime}}<t_{3 r}^{x^{\prime}}\right\} \exp \left(-\int_{0}^{T_{A}^{x^{\prime}}} \phi\left(\left|Y_{s}^{x^{\prime}}\right|\right) d s\right)\right] .
\end{aligned}
$$

In $\Delta_{2 a}$, the event in the indicator function belongs to $\mathcal{F}_{t_{3 r}^{x^{\prime}}}$, and so by the strong Markov property, $\left|Y_{t_{3 r}^{x^{\prime}}}^{x^{\prime}}\right| \leq 5 r \leq K$, monotonicity from Lemma 8.3, the coupling bound (8.34) with 
$\varepsilon=1 / 2$ and $\delta=3 r$, and Lemma 8.4,

$$
\begin{aligned}
\Delta_{2 a}\left(x, x^{\prime}, A\right) & =E\left[1\left\{t_{3 r}^{x}<S_{c}, t_{3 r}^{x} \leq t_{3 r}^{x^{\prime}}<T_{A}^{x^{\prime}}\right\} E_{\substack{Y^{x^{\prime}} \\
t_{3 r}^{\prime}}}\left[\exp \left(-\int_{0}^{T_{A}} \phi\left(\left|Y_{s}\right|\right) d s\right)\right]\right] \\
& \leq P\left(t_{3 r}^{x}<S_{c}\right) \Phi((K, 0), A) \\
& \leq C \frac{\log K}{\sqrt{K} \log \left(A^{2}\right)} .
\end{aligned}
$$

Finally, consider $\Delta_{2 b}$. Dropping the exponential and applying the strong Markov property to $Y^{x^{\prime}}$ at time $t_{3 r}^{x}$, we have

$$
\begin{aligned}
\Delta_{2 b}\left(x, x^{\prime}, A\right) & \leq P\left(t_{3 r}^{x}<S_{c}, t_{3 r}^{x} \leq T_{A}^{x^{\prime}}<t_{3 r}^{x^{\prime}}\right) \\
& =E\left[1\left\{t_{3 r}^{x}<S_{c} \wedge T_{A}^{x^{\prime}}\right\} P_{Y_{t_{3 r}^{\prime}}^{x^{\prime}}}\left(T_{A}<t_{3 r}\right)\right] .
\end{aligned}
$$

By Lemma 8.11(b), on the event $\left\{t_{3 r}^{x}<S_{c}\right\},\left|Y_{t_{3 r}^{2}}^{x^{\prime}}\right| \leq 2 K+10 r$. Let $K_{0}$ be large enough so that $K>K_{0}$ implies $K^{2}>(2 K / r)+10$, and assume additionally that $K>K_{0}$ and $A>(2 K+10 r) \vee r^{2}$. By the hitting probability bound (5.11), with $a=3 r$ we see that if $\left|Y_{t_{3 r}^{\prime}}^{x^{\prime}}\right|>3 r$, then

$$
P_{Y_{t_{3 r}^{\prime \prime}}^{x^{\prime}}}\left(T_{A}<t_{3 r}\right) \leq \frac{\log \left(\frac{2 K}{r}+10\right)}{\log \frac{A}{r}} \leq \frac{\log \left(K^{2}\right)}{\log (\sqrt{A})}=\frac{8 \log K}{\log \left(A^{2}\right)} .
$$

The same bound holds if $\left|Y_{T_{3 r}^{x}}^{x^{\prime}}\right| \leq 3 r$ because then the left-hand side is zero. Now the additional restrictions on $K, A$ can be dropped by replacing 8 with a larger constant $C$, so we may conclude that for $A, K$ as in the Lemma and on $\left\{t_{3 r}^{x}<S_{c}\right\}$,

$$
P_{Y_{t_{3 r}^{\prime}}^{x^{\prime}}}\left(T_{A}<t_{3 r}\right) \leq C \frac{\log K}{\log \left(A^{2}\right)} .
$$

Insert this into (8.45), apply Lemma 8.11(c) as before, to obtain

$$
\Delta_{2 b}\left(x, x^{\prime}, A\right) \leq P\left(t_{3 r}^{x}<S_{c}\right) \frac{C \log K}{\log \left(A^{2}\right)} \leq C \frac{\log K}{\sqrt{K} \log \left(A^{2}\right)} .
$$

Combine (8.43), (8.44) and (8.46) to complete the proof with $\varepsilon_{K}$ as claimed.

Theorem 8.13. For all $x \neq 0$ there exists $c_{\phi}(x)>0$ such that

$$
\lim _{A \rightarrow \infty} \log \left(A^{2}\right) \Phi(x, A)=c_{\phi}(x) .
$$

Proof. We may assume $x$ is on the positive $x_{1}$-axis, and for now that $|x|>3 r$. Assume $K>(|x|+2 r) \vee 2$ and $A>2 K+2 r$, put $x_{K}=(K, 0)$ and $\varepsilon_{K}=(\log K) / \sqrt{K}$ as in Lemma 8.12. By the strong Markov property,

$$
\Phi(x, A)=E_{x}\left[\exp \left(-\int_{0}^{T_{K}} \phi\left(Y_{s}\right) d s\right) \Phi\left(Y_{T_{K}}, A\right)\right] .
$$

By Lemma 8.12, noting that $\left|Y_{T_{K}}\right| \in[K, K+2 r]$,

$$
\left|\Phi\left(Y_{T_{k}}, A\right)-\Phi\left(x_{K}, A\right)\right| \leq C_{8.39} \frac{\varepsilon_{K}}{\log \left(A^{2}\right)} .
$$

Using this bound in (8.48) we obtain

$$
\Phi(x, A)=\Phi(x, K) \Phi\left(x_{K}, A\right)+\mathcal{E}_{1},
$$


where $\left|\mathcal{E}_{1}\right| \leq C \varepsilon_{K} / \log \left(A^{2}\right)$. Now consider $\Phi\left(x_{K}, A\right)$. On account of $\phi\left(Y_{s}^{x_{K}}\right)=0$ for $s>t_{3 r}$, (8.14) and the strong Markov property, we see that

$$
\begin{aligned}
\Phi\left(x_{K}, A\right) & =P_{x_{K}}\left(T_{A}<t_{3 r}\right)+E_{x_{K}}\left[1\left\{t_{3 r}<T_{A}\right\} E_{Y_{t_{3 r}}}\left[\exp \left(-\int_{0}^{T_{K}} \phi\left(Y_{s}\right) d s\right) \Phi\left(Y_{T_{K}}, A\right)\right]\right. \\
& =P_{x_{K}}\left(T_{A}<t_{3 r}\right)+\Phi\left(x_{K}, A\right) E_{x_{K}}\left[1\left\{t_{3 r}<T_{A}\right\} \Phi\left(Y_{t_{3 r}}, K\right)\right]+\mathcal{E}_{2},
\end{aligned}
$$

where $\left|\mathcal{E}_{2}\right| \leq C \frac{\varepsilon_{K}}{\log \left(A^{2}\right)}$ by (8.49). If we set

$$
\alpha\left(x_{K}, A\right)=E_{x_{K}}\left[1\left\{t_{3 r}<T_{A}\right\} \Phi\left(Y_{t_{3 r}}, K\right)\right]<1
$$

then (8.51) can be rearranged to obtain

$$
\Phi\left(x_{K}, A\right)=\frac{P_{x_{K}}\left(T_{A}<t_{3 r}\right)+\mathcal{E}_{2}}{1-\alpha\left(x_{K}, A\right)} .
$$

Plugging this into (8.50) gives

$$
\Phi(x, A)=\Phi(x, K) \frac{P_{x_{K}}\left(T_{A}<t_{3 r}\right)+\mathcal{E}_{2}}{1-\alpha\left(x_{K}, A\right)}+\mathcal{E}_{1},
$$

and thus

$$
\log \left(A^{2}\right) \Phi(x, A)=\frac{\Phi(x, K)}{1-\alpha\left(x_{K}, A\right)} \log \left(A^{2}\right) P_{x_{K}}\left(T_{A}<t_{3 r}\right)+\mathcal{E}_{3},
$$

where $\left|\mathcal{E}_{3}\right| \leq C \varepsilon_{K} /\left(1-\alpha\left(x_{K}, A\right)\right)$.

Now consider $\alpha\left(x_{K}, A\right)$. By recurrence, $1\left\{t_{3 r}<T_{A}\right\} \rightarrow 1 P_{x_{K}}$-a.s. as $A \rightarrow \infty$, which implies the limit

$$
\alpha\left(x_{K}, \infty\right):=\lim _{A \rightarrow \infty} \alpha\left(x_{K}, A\right)=E_{x_{K}}\left[\Phi\left(Y_{t_{3 r}}, K\right)\right]
$$

exists. By monotonicity (Lemma 8.3), $\left|Y_{t_{3 r}}\right| \leq 3 r$, and recurrence, we have $\alpha\left(x_{K}, \infty\right) \leq$ $\Phi((3 r, 0), K) \rightarrow 0$ as $K \rightarrow \infty$. If we let $K_{0}$ satisfy $\alpha\left(x_{K}, \infty\right) \leq 1 / 2$ for all $K \geq K_{0}$, then $\left|\mathcal{E}_{3}\right| \leq 2 C \varepsilon_{K}$. Thus, assuming in addition that $K>K_{0}$, we have from (8.53) and the above,

$$
\limsup _{A \rightarrow \infty}\left|\log \left(A^{2}\right) \Phi(x, A)-\Phi(x, K) \frac{\log \left(A^{2}\right) P_{x_{K}}\left(T_{A}<t_{3 r}\right)}{1-\alpha\left(x_{K}, A\right)}\right| \leq C \varepsilon_{K} .
$$

By (5.12), $\left(\log A^{2}\right) P_{x_{K}}\left(T_{A}<t_{3 r}\right)$ is bounded above for large $A$ and approaches $p\left(3 r, x_{K}\right):=2\left(\log \left|x_{K}\right|-E_{x_{K}}\left[\log \left|Y_{t_{3 r}}\right|\right]\right)>0$ as $A \rightarrow \infty$. It follows from (8.54) that

$$
\limsup _{A \rightarrow \infty}\left|\log \left(A^{2}\right) \Phi(x, A)-\Phi(x, K) \frac{p\left(3 r, x_{K}\right)}{1-\alpha\left(x_{K}, \infty\right)}\right| \leq C \varepsilon_{K} .
$$

The fact that $\varepsilon_{K} \rightarrow 0$ as $K \rightarrow \infty$ now implies $A \mapsto \log \left(A^{2}\right) \Phi(x, A)$ as $A \rightarrow \infty$ is Cauchy, hence there exists $c_{\phi}(x) \in[0, \infty)$ such that $\lim _{A \rightarrow \infty} \log \left(A^{2}\right) \Phi(x, A)=c_{\phi}(x)$.

To check that $c_{\phi}(x)>0$, note that (for $K$ large as above) (8.55) implies

$$
\liminf _{A \rightarrow \infty} \log \left(A^{2}\right) \Phi(x, A) \geq p\left(3 r, x_{K}\right) \frac{\Phi(x, K)}{1-\alpha\left(x_{K}, \infty\right)}-C \varepsilon_{K} \geq p\left(3 r, x_{K}\right) \Phi(x, K)-C \varepsilon_{K} .
$$

The right-hand side above is positive if, for large $K, \Phi(x, K) / \varepsilon_{K} \rightarrow \infty$ as $K \rightarrow \infty$ (note that $p\left(3 r, x_{K}\right) \rightarrow \infty$ as $\left.K \rightarrow \infty\right)$. Using $\Phi(x, K) \geq P_{x}\left(T_{K}<t_{3 r}\right)$, we have

$$
\frac{\Phi(x, K)}{\varepsilon_{K}} \geq \frac{\sqrt{K}}{\log K} P_{x}\left(T_{K}<t_{3 r}\right)=\frac{\sqrt{K}}{(\log K)^{2}} \log (K) P_{x}\left(T_{K}<t_{3 r}\right) \rightarrow \infty \text { as } K \rightarrow \infty
$$

by Lemma 5.3. Hence, $c_{\phi}(x)>0$. 
Finally, suppose $0<|x| \leq 3 r$. By the strong Markov property, for $A>6 r$,

$$
\Phi(x, A)=E_{x}\left[\exp \left(-\int_{0}^{T_{4 r}} \phi\left(Y_{s}\right) d s\right) \Phi\left(Y_{T_{4 r}}, A\right)\right] .
$$

By monotonicity (Lemma 8.3) and Lemma 8.4,

$$
\log \left(A^{2}\right) \Phi\left(Y_{T_{4 r}}, A\right) \leq \log \left(A^{2}\right) \Phi((6 r \vee 2,0), A) \leq C_{8.15} \log (6 r \vee 2),
$$

and also converges to $c_{\phi}\left(Y_{T_{4 r}}\right)>0$ a.s. as $A \rightarrow \infty$, by the above and $\left|Y_{T_{4 r}}\right|>3 r$. Apply bounded convergence to obtain existence of the limit

$$
\lim _{A \rightarrow \infty} \log \left(A^{2}\right) \Phi(x, A)=E_{x}\left[\exp \left(-\int_{0}^{T_{4 r}} \phi\left(Y_{s}\right) d s\right) c_{\phi}\left(Y_{T_{4 r}}\right)\right]>0 .
$$

This completes the proof of (8.47).

Proof of Proposition $1.1(d=2)$. Let $\phi(x)=k(x)$, so $\Phi(x, A)=E_{x}\left[\exp \left(-\int_{0}^{T_{A}} k\left(Y_{s}\right) d s\right)\right]$. By Theorem 8.13, for $x \neq 0$, the positive limit $c_{k}(x)=\lim _{A \rightarrow \infty} \log \left(A^{2}\right) \Phi(x, A)$ exists. By Lemma 8.4, there is a constant $C>0$ such that for all large $A,\left(\log A^{2}\right) \Phi(x, A) \leq C$ for all $|x| \leq 2 r$. Therefore, by bounded convergence, if $Y_{0}=\bar{U}$, the limit

$$
\lim _{A \rightarrow \infty}\left(\log \left(A^{2}\right)\right) E_{Y_{0}}\left[\exp \left(-\int_{0}^{T_{A}} k\left(Y_{s}\right) d s\right)\right]=\lim _{A \rightarrow \infty} E\left(\log \left(A^{2}\right) \Phi\left(Y_{0}, A\right)\right)=E\left(c_{k}\left(Y_{0}\right)\right)>0
$$

exists. That is, (8.3) holds for $Y_{0}=\bar{U}$, and by Proposition 8.1, the limit

$$
\lim _{t \rightarrow \infty}(\log t) P_{Y_{0}}(\kappa>t)=E\left(c_{k}\left(Y_{0}\right)\right)>0
$$

exists, which is exactly (1.16).

Remark 8.14. Theorem 8.13 is proved for continuous time rate $2 \rho\left|B_{r}\right|$ random walks $Y_{t}$ with step distribution (1.3). The result is needed in our proof of Proposition 1.1 because the dual particle difference $\tilde{\xi}_{t}$ behaves like $Y_{t}$ when $\left|\tilde{\xi}_{t}\right|>2 r$ in our fixed radius case of the SLFV. With a view to the variable radius case in (1.17), we remark that Theorem 8.13 holds for more general radially symmetric step distributions. Assume $r_{\max }>0$ and suppose $\nu$ is a probability measure on $\left[0, r_{\max }\right]$ with a bounded density such that $\int_{0}^{r_{\max }} \rho^{-2} \nu(d \rho)<\infty$, and consider the step distribution on $\mathbb{R}^{2}$ given by

$$
\int_{0}^{r_{\max }} \nu(d \rho) P(\rho U \in \cdot),
$$

where $U$ is uniform on $B_{1}(0)$. A bit of calculus shows that in the variable radius case (1.17), $\tilde{\xi}_{t}$ behaves like a fixed rate random walk with step distribution satisfying (8.57) for appropriate $\nu$ when $\left|\tilde{\xi}_{t}\right|>2 r_{\max }$. Theorem 8.13 holds when $Y_{t}$ is a continuous time rate $\lambda>0$ with this step distribution (8.57). The modifications needed to prove this are minor. Of course this will not immediately give Proposition 1.1 as we no longer have an identity like (8.1) which arose from our time-change representation for the difference of the dual components.

Acknowledgments. We thank Sarah Penington for answering our queries on [14]. Some of this work was carried out while the first author was visiting the Mathematics Department at the University of British Columbia, or while the second author was visiting the Mathematics Department at Syracuse University. We thank both Institutions for their hospitality. 


\section{Convergence of SLFV to SBM}

\section{References}

[1] Arratia, R. Coalescing Brownian motions on $\mathbb{R}$ and the voter model on $\mathbb{Z}$. Uncompleted manuscript, 32 pages, based on thesis and extensions (1981). Available on request to rarratia@math.usc.edu.

[2] Barton, N.N., Etheridge, A.M., and Veber, A. (2010). A new model for spatial evolution in a continuum. Electron. J. Probab. 15 162-216, MR-2594876

[3] Bhattacharya, R. N., and Ranga Rao, R. Normal Approximation and Asymptotic Expansions. John Wiley and Sons, Inc., 1976. MR-3396213

[4] Burkholder, D. L. (1973). Distribution function inequalities for martingales. Ann. Probab. 1 19-42. MR-0365692

[5] Chetwynd-Diggle, J. A., and Etheridge, A. M. (2018). SuperBrownian motion and the spatial Lambda-Fleming-Viot process. Electron. J. Probab. 23:1-36. MR-3835477

[6] Cox, J. T., Durrett, R., and Perkins, E. (2000). Rescaled voter models converge to superBrownian motion. Ann. Probab. 28 185-234. MR-1756003

[7] Cox, J. T., and Perkins, E. (2005). Rescaled Lotka-Volterra models converge to Super-Brownian motion. Ann. Probab. 33 904-947. MR-2135308

[8] Cox, J. T., and Perkins, E. (2008). Renormalization of the two-dimensional Lotka-Volterra model. Ann. Appl. Probab. 18 747-812. MR-2399711

[9] Dawson, D.A. (1975). Stochastic evolution equations and related measure processes. J. Multivariate Analysis 3:1-52. MR-0388539

[10] Dawson, D.A. (1993). Measure-valued Markov processes. Lecture Notes Math. 1541:1-260. Springer, Berlin. MR-1242575

[11] Durrett, R. and Perkins, E. (1998). Rescaled contact processes converge to super-Brownian motion for $d \geq 2$. Prob. Theory Rel. Fields. 114 309-399. MR-1705115

[12] Etheridge, A. M. (2008). Drift, draft and structure: some mathematical models of evolution. Banach Center Publ. 90:121-144. MR-2433141

[13] Etheridge, A. M. (2000). An Introduction to Superprocesses, Univ. Lect. Series 20, Amer. Math. Soc., Providence, Rhode Island. MR-1779100

[14] Etheridge, A. M., Freeman, N., Penington, S., and Straulino, D. (2017). Branching Brownian motion and selection in the spatial $\Lambda$-Fleming-Viot Process. Ann. Probab. 27 2605-2645. MR-3719942

[15] Etheridge, A. M., Véber, A., and Yu, F. (2018). Rescalings of the spatial Lambda-Fleming process with selection. Math. Arxiv: $1406.5884 \mathrm{v} 2$.

[16] Hofstad, R. v.d., and Sakai, A. (2010). Convergence of the critical finite-range contact process to super-Brownian motion above the upper critical dimension: the higher-point functions. Electron. J. Probab. 15, 801-894. MR-2653947

[17] Hofstad, R. v.d. and Slade, G. (2003). Convergence of critical oriented percolation to superBrownian motion above $4+1$ dimensions. Ann. Inst. H. Poincaré Probab. Statist. 39 413-485. MR-1978987

[18] Hofstad, R. v.d., Holmes, M., and Perkins, E. (2017). A criterion for convergence to superBrownian motion on path space. Ann. Prob. 45 278-376. MR-3601651

[19] Ethier, S. and Kurtz, T. Markov Processes. Characterization and Convergence. John Wiley \& Sons, Inc., 1986. MR-1567615

[20] Jacod, J and Shiryaev, A.N. Limit Theorems for Stochastic Processes, 2nd ed. Grundlehren der Mathematischen Wissenschaften, 2003. MR-1943877

[21] Mueller, C. and Tribe, R. (1995). Stochastic p.d.e.'s arising form the long range contact and long range voter processes. Probab. Th. Rel. Fields 102:519-545 (1995). MR-1346264

[22] Perkins, E. (2002). Dawson-Watanabe superprocesses and measure-valued diffusions. Lecture Notes Math. 1781:125-324. Springer, Berlin. MR-1915445

[23] Presutti, El, and Spohn, H. (1983). Hydrodynamics of the voter model. Ann. Probab. 11 867-875. MR-0714951 


\section{Convergence of SLFV to SBM}

[24] Ren, Yaofeng and Tian, Fan-Ji. (2003). On the Rosenthal's inequality for locally square integrable martingales. Stoch. Process. Appl. 104 107-116. MR-1956474

[25] Veber, A. and Wakolbinger, A. (2015). The spatial Lambda-Fleming-Viot process and a lookdown representation. Ann. Inst. H. Poincaré, 51:570-598. MR-3335017

[26] Watanabe, S. (1968). A limit theorem of branching processes and continuous state branching processes. J. Math. Kyoto U. 8:141-167. MR-0237008 